\title{
Decoding the alcohol-IAT : the implicit association test as a measure of individual differences in implicit preferences for alcohol
}

Citation for published version (APA):

Houben, K. M. P. I. (2007). Decoding the alcohol-IAT : the implicit association test as a measure of individual differences in implicit preferences for alcohol. [Doctoral Thesis, Maastricht University]. Datawyse / Universitaire Pers Maastricht. https://doi.org/10.26481/dis.20070420kh

Document status and date:

Published: 01/01/2007

DOI:

10.26481/dis.20070420kh

Document Version:

Publisher's PDF, also known as Version of record

Please check the document version of this publication:

- A submitted manuscript is the version of the article upon submission and before peer-review. There can be important differences between the submitted version and the official published version of record. People interested in the research are advised to contact the author for the final version of the publication, or visit the DOI to the publisher's website.

- The final author version and the galley proof are versions of the publication after peer review.

- The final published version features the final layout of the paper including the volume, issue and page numbers.

Link to publication

\footnotetext{
General rights rights.

- You may freely distribute the URL identifying the publication in the public portal. please follow below link for the End User Agreement:

www.umlib.nl/taverne-license

Take down policy

If you believe that this document breaches copyright please contact us at:

repository@maastrichtuniversity.nl

providing details and we will investigate your claim.
}

Copyright and moral rights for the publications made accessible in the public portal are retained by the authors and/or other copyright owners and it is a condition of accessing publications that users recognise and abide by the legal requirements associated with these

- Users may download and print one copy of any publication from the public portal for the purpose of private study or research.

- You may not further distribute the material or use it for any profit-making activity or commercial gain

If the publication is distributed under the terms of Article $25 \mathrm{fa}$ of the Dutch Copyright Act, indicated by the "Taverne" license above, 


\section{Decoding the Alcohol-IAT}

The Implicit Association Test as a Measure of Individual Differences in Implicit Preferences for Alcohol 


\section{Colophon}

Graphic design by Jochen Punie \& Katrijn Houben Production: Datawyse | Universitaire Pers Maastricht

(c) Katriin Houben, Maastricht 2007

ISBN 978-90-5278-616-2

The studies presented in this dissertation were funded by the Dutch National Science Foundation (NWO, VIDI grant 452.02.005). 


\section{Decoding the Alcohol-IAT The Implicit Association Test as a Measure of Individual Differences in Implicit Preferences for Alcohol}

\section{PROEFSCHRIFT}

ter verkrijging van de graad van doctor aan de Universiteit Maastricht, op gezag van de Rector Magnificus, prof. mr. G.P.M.F. Mols, volgens het besluit van het College van Decanen, in het openbaar te verdedigen op vrijdag 20 april 2007 om 14.00 uur

door

Katrijn Marie Patricia Irma Houben

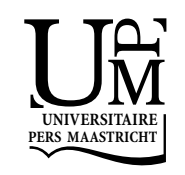




\section{PROMOTORES}

Prof. dr. A.T.M. Jansen

Prof. dr. R.W. Wiers

\section{BEOORDELINGSCOMMISSIE}

Prof. dr. A. Arntz (voorzitter)

Prof. dr. J. De Houwer (Universiteit Gent, België)

Prof. dr. R. Engels (Radboud Universiteit Nijmegen)

Prof. dr. P.J. de Jong (Rijksuniversiteit Groningen)

Dr. F.T.Y. Smulders 


\section{Contents}

Chapter 1 General Introduction

PART I RECODING INFLUENCES ON PERFORMANCE IN THE ALCOHOL-IAT

Chapter 2 Testing the Salience Asymmetry Account of the Alcohol-IAT

Chapter 3 Eliminating Recoding in the Alcohol-IAT: An Application of the IAT-RF

Chapter 4 Are Drinkers Implicitly Positive about Drinking Alcohol?

Personalizing the Alcohol-IAT to Reduce Negative Extrapersonal

Contamination

Chapter 5 Personalizing the Alcohol-IAT with Individualized Stimuli:

Relationship with Drinking Behavior and Drinking-Related Problems

PART ॥ BIPOLAR TARGET AND ATTRIBUTE DIMENSIONS IN THE ALCOHOL-IAT: DOUBLE JEOPARDY

Chapter 6 Assessing Implicit Alcohol Associations with the Implicit Association Test: Fact or Artifact?

Chapter 7 Single-Target Implicit Preferences for Beer as Determinants of Consumption

Chapter 8 Implicit Positive Alcohol Attitudes as Determinants of Alcohol Consumption: Beyond the Soda Contrast in the IAT

Chapter 9 General Discussion

Summary

Samenvatting

References

Dankwoord 


$$
\text { D) }
$$




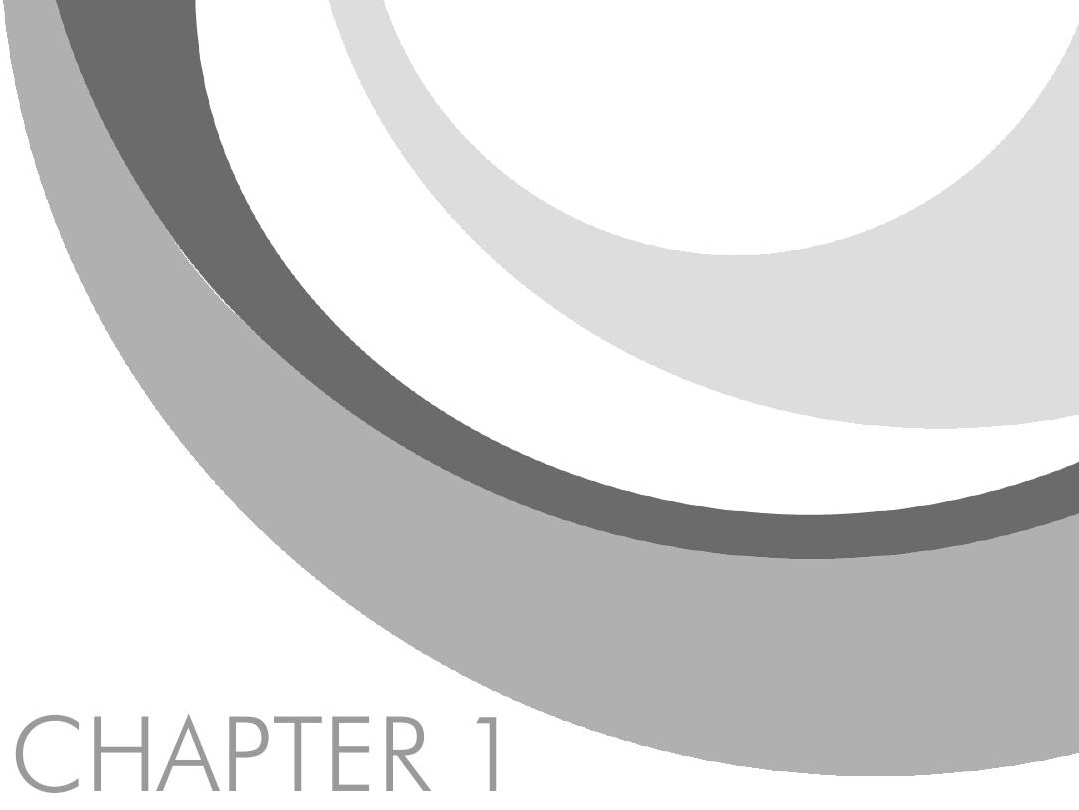

General Introduction 
There has been a long tradition in psychology aimed at registering cognitive processes that play a role in the etiology and maintenance of addictive behaviors in an attempt to explain and control substance use and abuse. Until recently, most research on cognitive processes involved in addictive behaviors, including alcohol abuse, has centered on the assessment of cognitive processes using direct, self-report measures. Most research using self-report measures of alcohol-related cognitive processes has been focused on the assessment of drinking motives (e.g., "How often do you drink alcohol because it is fun?"; Cooper, 1994; Cooper, Frone, Russell, \& Mudar, 1995) and alcohol outcome expectancies (e.g., "Drinking alcohol makes me feel good"; Brown, Goldman, Inn, \& Anderson, 1980; Goldman, Del Boca, \& Darkes, 1999). Together, these studies identified three basic types of alcohol-related cognitions that are involved in alcohol abuse: positive reinforcement (i.e., drinking to enhance positive affect), negative reinforcement (i.e., drinking to decrease negative affect), and negative expectancies (i.e., drinking is expected to result in negative outcomes) (see also Wiers, Houben, Smulders, Conrod, \& Jones, 2006).

Although research within the framework of drinking motives and alcohol expectancy theory has been fruitful in identifying possible cognitive processes underlying alcohol abuse, it may be questioned to what extent research using self-report methods can fully reveal the fundamental cognitive processes involved in addictive behaviors. Importantly, self-report measures are sensitive to social desirability factors, including impression management, and self-deception, which refer to a tendency to respond to self-report measures in a manner that makes the respondent look good rather than in an accurate and truthful way (e.g., Holtgraves, 2004; Paulhus, 1984). Hence, self-report measures are dependent on what people are willing to report regarding the cognitive processes that steer their behavior. In addition, it has been suggested that people may not always be able to report on the cognitive processes underlying their behavior (Nisbett \& Wilson, 1977).

\section{DUAL-PROCESS THEORIES OF DRINKING BEHAVIOR}

Many psychological theories explaining human behavior, including addictive behaviors, are rooted in rational decision theory (e.g., Aizen, 1991; Bandura, 1997) and have in common the assumption that people will do what they believe is good for them. However, it is obvious that people often act in ways that are inconsistent with this rational model and addictive behavior is one of these instances of irrational behavior. Paradoxically, people are often very aware of the harmful effects of their substance abuse and yet they continue this detrimental behavior.

Irrational behavior like substance abuse may be better understood from a dualprocess point of view. The core tenet of dual-process theories is that behavior is determined by the interplay of two qualitatively different systems: a fast associative "impulsive" system in which activation automatically spreads between associated contents, 
and a slow symbolic "reflective" system that relies on rule-based or controlled processing (e.g., Deutsch \& Strack, 2006; Evans, 2003; Evans \& Coventry, 2006; Strack \& Deutsch, 2004). Moreover, it was suggested that, in the impulsive system, the amygdala is the critical neural structure which triggers affective signals of immediate outcomes of an option, whereas the reflective system is dependent on prefrontal neural structures that trigger affective signals of long-term outcomes of an option (Bechara, 2005; Bechara, Noel, \& Crone, 2006).

While the reflective system is assumed to determine behavior through deliberative decision making, the impulsive system can activate behavioral schemata automatically through spreading activation or by influencing the reflective system (Bechara, 2005; Bechara et al., 2006; Deutsch \& Strack, 2006; Strack \& Deutsch, 2004). Importantly, the impulsive and reflective system can trigger simultaneous, conflicting signals, but ultimately, behavioral decisions are determined by the relative strengths of impulsive and reflective processes so that stronger processes gain advantage over weaker ones (Bechara, 2005, Bechara et al., 2006; Deutsch \& Strack, 2006; Strack \& Deutsch, 2004). Importantly, in addiction, the reflective system appears unable to dominate the impulsive system either due to a dysfunctional reflective system that is unable to inhibit dominant automatic responses triggered by impulsive system, and/or because of a hyperactive impulsive system that hijacks the cognitive resources that are needed for the normal operation of the reflective system (Bechara, 2005; Bechara et al., 2006).

\section{IMPLICIT MEASURES OF ALCOHOL-RELATED COGNITIONS}

Evidently, there is not only a need for measures that can be used to scrutinize the reflective system but also for measures that can tap the impulsive system in order to fully comprehend the cognitive processes that determine alcohol use and abuse. It can be argued that self-report measures of alcohol-related cognitive processes may not be appropriate for examining impulsive or automatic cognitive processes since they present ample opportunity for the reflective system to influence responding while at the same time, respondents may lack introspective access with respect to the origins of affective reactions that are automatically triggered in the impulsive system as well as the automatic influence of such affective reactions on behavior. For this reason, the increased understanding of human behavior in terms of two semi-independent systems has gone hand in hand with the development of a new class of assessment methods that should be better suited to tap automatic, impulsive cognitive processes. More specifically, researchers have turned to measures of cognitive processes that infer cognitive processes from behavior other than self-report. These measures are often referred to as "implicit" measures as opposed to selfreport measures which are typically termed "explicit" measures. 


\section{Where is the Implicit?}

Despite the immense popularity of so-called implicit measures, the denotation of the term "implicit" remains confusing. Some have argued that the term refers to the measurement procedure (Fazio \& Olson, 2003), while others believe that implicit measures have privileged access to automatic cognitive processes and thus tap different underlying cognitive processes than self-report measures (e.g., Cunningham, Preacher, \& Banaji, 2001; Stacy, 1997; Wilson, Lindsey, \& Schooler, 2000). According to De Houwer (2006) these latter two views of implicit measures actually refer to different things, namely the measurement procedure and the outcome of the measurement procedure, respectively.

As De Houwer (2006) pointed out, most definitions of implicit measures that are found in recent psychological literature actually refer to functional properties of measurement outcomes, and more specifically to the nature of the processes through which to-be-measured cognitions are translated into measurement outcomes, rather than properties of measurement procedures (which are simply a set of objective guidelines). De Houwer (2006) further argues that an implicit measure is a measurement outcome that reflects the to-be-measured construct by virtue of processes that have certain functional properties that do not necessarily have to co-occur. These functional properties of implicit measures include that the processes that translate cognitive constructs into the measurement outcome operate even though participants are unaware of the fact that the measurement outcome measures something, even when participants are unaware of the cognitions that are measured, and despite efforts to control the measurement outcome. Moreover, with respect to the precise functional properties that should be considered when defining a measurement outcome as implicit, De Houwer (2006) suggested that it might be useful to replace the term "implicit" with the term "automatic" since the term "automatic" is often used to define the nature of processes and can therefore easily be used to describe the processes underlying measurement outcomes, and because there is a long tradition of research on the features that are linked to automaticity. When the term "implicit" is considered as a synonym for the concept "automatic", implicit measures can be defined as measurement outcomes that reflect a certain cognitive construct due to processes that are uncontrolled, unintentional, goal-independent, autonomous, purely stimulus-driven, unconscious, efficient, or slow (De Houwer \& Moors, in press). Importantly, each of these functional properties should be examined separately in order to define in what sense a measurement outcome is an implicit measure of a certain cognitive construct (i.e., in what sense the processes through which a measurement outcome reflects a certain cognitive construct can be characterized as automatic) (De Houwer, 2006, in press).

In contrast, when the term measure is used to denote measurement procedures, we are in fact referring to a set of objective guidelines and it makes no sense to use the adjective "implicit" since there is nothing unconscious, uncontrollable or automatic about measurement procedures (De Houwer, 2006, in press). Further, self-report measures and 
so-called implicit measurement procedures can be easily distinguished by virtue of the nature of their objective guidelines. Self-report measures are direct in the sense that participants are asked to respond to questions about the cognitive processes of interest. In contrast, so-called implicit measurement procedures infer cognitive processes indirectly from performance other than self-assessment. Hence, De Houwer (2006) argues that measurement procedures can be better characterized as direct or indirect measures and that the direct or indirect nature of measurement procedures can be objectively determined based on its objective properties. Following De Houwer (2006), the terms "direct" and "indirect" will used be here to denote measurement procedures, while the terms "implicit" and "automatic" will be used when referring to cognitive processes that are assessed with indirect measures whereas the term "explicit will be used to refer to cognitive processes measured with direct measures.

\section{Indirect Measures of Alcohol-Related Cognitions}

Word Association Measures. Word association measures (e.g., Stacy, 1995, 1997; Stacy, Ames, Sussman, \& Dent, 1996; Stacy, Leigh, \& Weingardt, 1994) are designed to measure substance-related associations by instructing participants to respond to a cue word or phrase as quickly as possible with the first response that comes to mind. As such, word association measures include measures of associative links between cues and substance use (e.g., draft: ___ ) and between outcomes and substance use (e.g., having fun: _ Importantly, results with word association measures have been demonstrated be related to substance use (e.g., Stacy et al., 1994; Stacy, 1995; Stacy et al., 1996), and to account for variability in substance use above self-report measures of alcohol-related cognitions (Stacy, 1997).

Priming Paradigms. Sequential priming techniques are particularly useful for studying the associative structures of representations in the impulsive system (e.g., Bargh \& Chartrand, 2000). In semantic priming, associations are assessed between two different concepts and in affective priming associations are assessed between a concept and its evaluation. In both priming procedures, the presentation of a prime word or phrase, assumed to provide a semantic or evaluative context, is followed by the presentation of a target word to which a response has to be generated. For both semantic and affective priming procedures, priming effects are indicated by an interaction between prime and target valence or meaning, showing faster performance when prime and target are congruent than when they are incongruent.

Hill and Paynter (1992) found that alcohol-related words (e.g., liqueur) but not unrelated neutral words (e.g., bulk) primed responding to alcohol-related targets (e.g., bottle) in alcohol-dependent drinkers but not in non-dependent drinkers, suggesting that alcohol-related concepts are more strongly interrelated in alcohol-dependent drinkers than 
in non-dependent drinkers. Weingardt, Stacy, and Leigh (1996) demonstrated faster responses to alcohol-related targets when primed with positive outcomes of drinking alcohol (e.g., "they had fun after they had the-") than when primed with neutral phrases (e.g., "they said it was the-") and that the degree of priming was related to drinking behavior. Hence, these results demonstrate that increased alcohol consumption is related to greater activation of alcohol-related concepts following priming with positive outcomes (i.e., positive reinforcement), which is consistent with results from alcohol expectancy research (e.g., Rather, Goldman, Roehrich, \& Brannick, 1992). Zack and co-workers demonstrated that negative primes consistently prime alcohol-related concepts in problem drinkers with high levels of psychiatric distress (Zack, Toneatto, \& MacLeod, 1999) as well as in young drinkers for whom the degree of priming was related to the severity of alcoholrelated problems (Zack, Poulos, Fragopoulos, \& Macleod, 2003). These results are in line with research that has demonstrated that negative reinforcement motivations for drinking are related to alcohol-related problems (e.g., Cooper et al., 1995). Finally, Ostafin, Palfai, and Wechsler (2003) calculated facilitation scores for approach and avoidance targets that were preceded by either an alcohol-related prime or a neutral prime. Results showed that alcohol binge episodes and alcohol-related problems were related to weak associations between alcohol and avoidance tendencies, and unrelated to associations between alcohol and approach tendencies.

The Implicit Association Test. The Implicit Association Test (IAT; Greenwald, McGhee, \& Schwartz, 1998) is a double categorization task that requires participants to classify stimuli into four different categories with two response keys. Typically, two categories represent target concepts (e.g., alcohol versus soft drinks) and two categories correspond to the poles of an attribute dimension (e.g., positive versus negative). During the critical trials of the IAT, the target and attribute categories are assigned to two response keys in two different combinations. During one combination task, for example, participants have to classify both alcohol and negative words with one response key and soft drinks and positive words with the other. During the reversed combination task, alcohol and positive are assigned to the same key and soft drinks and negative to the other. Because performance will be better when concepts that are associated in memory are classified with the same response key (compatible), than when concepts sharing a response key are not or only weakly associated (incompatible), one of these combinations typically leads to faster and more accurate performance compared to the other. This performance difference is referred to as the IAT effect and is assumed to reflect the strength of implicit associations between the target concepts and the evaluative attribute categories (Greenwald et al., 1998).

Wiers, van Woerden, Smulders, \& de Jong (2002) were the first to apply the IAT in order to measure implicit alcohol associations. Specifically, alcohol associations were assessed in both heavy and light drinkers' with the IAT in two dimensions: valence and 
arousal. Both heavy and light drinkers displayed negative evaluative associations with alcohol compared with soft drinks (soda). In addition, heavy drinkers also demonstrated associations between alcohol and arousal while this effect was absent in light drinkers. These findings have been replicated in a sample of heavy drinkers (Wiers, van de Luitgaarden, van den Wildenberg, \& Smulders, 2005) and in a clinical sample of heavy drinkers (De Houwer, Crombez, Koster, \& De Beul, 2004). Further, Palfai and Ostafin (2003) modified the IAT by replacing the valence categories with approach and avoidance attributes. Results demonstrated that alcohol is associated with approach motivations (or action tendencies) in hazardous drinkers. Further, stronger alcohol-approach associations significantly correlated with urge to drink and arousal-reactivity in anticipation of alcohol consumption. Wiers et al. (2002) as well as Palfai and Ostafin (2003) suggested that arousal and approach associations with alcohol in heavy drinkers could be linked to the incentive-sensitization theory (Robinson \& Berridge, 1993, 2003) which states that activation of a sensitized "wanting" system automatically increases attention, arousal, and approach responses. Moreover, it was recently argued that incentive sensitization belongs to the impulsive system and might reflect hyperactivity of the impulsive system (Bechara et al., 2006; Deutsch \& Strack, 2006).

The Extrinsic Affective Simon Task. The Extrinsic Affective Simon Task (EAST; De Houwer, 2003) is a categorization task that allows the measurement of associations with one target concept within one task (rather than through a comparison of two combination tasks as in the IAT). In the EAST, attribute words (e.g., positive and negative) are typically classified by meaning using two response keys. Because of these task instructions, the response keys are assumed to become extrinsically associated with the meaning of the attributes assigned to them, creating for example an extrinsically positive and an extrinsically negative response key. In contrast, target words (e.g., alcohol, soda), are classified with respect to an irrelevant stimulus property (e.g., color, shape) with the same two response keys. Participants, for example, may be instructed to respond with one key to positive words as well as alcohol and soda stimuli that are presented in blue, and to respond with the other key to negative words as well as alcohol and soda targets that are presented in green. Subsequently, implicit evaluative associations with alcohol are defined as the performance difference between giving an extrinsically positive response to (blue) alcohol targets and giving an extrinsically negative response to (green) alcohol targets.

In a recent study, De Houwer et al. (2004) assessed alcohol associations with an EAST in a sample of patients. Alcoholics were more accurate in responding to soda stimuli with an extrinsically positive response than with an extrinsically negative response, but showed no performance difference with respect to alcohol stimuli. These results suggest that participants had a favorable attitude towards soft drinks and a neutral or possibly an ambivalent attitude towards alcohol. Further, de Jong, Wiers, van den Braak, and Huijding (in press) found similar results with the EAST, demonstrating positive evaluative 
associations with soda whereas results with respect to alcohol reflected neutral or ambivalent evaluative associations. Moreover, de Jong et al. (in press) also demonstrated that evaluative associations with alcohol were positively related to alcohol use and alcohol-related problems and even predicted behavior above self-reported attitudes. Finally, De Houwer and De Bruycker (in press) created a new variant of the EAST, the IDEAST, which forced participants to process the target stimuli. In the EAST, the meaning of target stimuli is irrelevant and should even be ignored since the correct response depends on the color in which the stimuli are presented. However, in order for the target concepts to have an influence on performance, it is necessary that the meaning of target concepts is processed. Therefore, in the ID-EAST, target and attribute stimuli are both presented in uppercase and lowercase letters and attribute stimuli are classified based on their meaning, whereas target stimuli are classified based on whether they are presented in uppercase or lowercase letters. Importantly, this procedure ensures that participants have to process target concepts since participants first have to identify stimuli as attributes or targets before they can select the correct response. De Houwer and De Bruycker (in press) demonstrated that heavy drinkers had positive evaluative associations with the target beer while light drinkers tended to show negative evaluative associations with beer. In addition, positive evaluative associations with beer predicted alcohol consumption above selfreported attitudes toward beer.

\section{Can Indirect Measurement Procedures Provide Implicit Measures?}

Clearly, indirect measures can be easily distinguished from direct measures based on their procedural guidelines. Further, indirect measures might be able to provide implicit measures in the sense that the outcomes of indirect measures reflect cognitive constructs because of processes that possess functional properties typical of automatic processes. However, whether or not an indirect measure is also an implicit measure needs to be empirically established based on features of automaticity that are related to goals (i.e., uncontrolled, unintentional, autonomous, purely stimulus-driven, goal-independent), unconsciousness, and efficiency (i.e., efficient and fast) (De Houwer, 2006; De Houwer \& Moors, in press).

To say that a measure is implicit in the sense that it is uncontrolled can refer either to the fact that the measurement outcome reflects the to-be-measured cognitive construct even though the goal to express the construct in the outcome is absent (i.e., unintentional) or to the fact that participants cannot control the translation of the to-be-measured construct into the measurement outcome (De Houwer \& Moors, in press). Moreover, a measure that is uncontrolled in both these senses can be referred to as autonomous. Studies that have examined whether indirect measures can provide an implicit measure in the sense that it is uncontrolled have concentrated mainly on whether participants are able to fake the measurement outcome (i.e., change, stop or avoid the translation of the to-be- 
measured construct in the measurement outcome). With respect to the IAT, studies have demonstrated that participants were unable to fake their IAT scores so that they would appear more positive toward homosexuals (Banse, Seise, \& Zerbes, 2001), present themselves as not shy (Asendorpf, Banse \& Mücke, 2002), or make a good impression (Egloff \& Schmukle, 2002). Importantly, Kim (2003) demonstrated that participants were unable to fake their IAT scores unless they were given practice with the task and specific instructions on how to fake the IAT. In line with these findings, Steffens (2004) as well as Fiedler and Bluemke (2005) demonstrated that prior experience with the IAT is crucial for being able to fake IAT scores. Thus, these results indicate that faking the IAT is possible but only given experience with the task. Up to now, no studies have yet examined the extent to which word association tasks, priming effects, and the EAST are susceptible to faking. However, it seems reasonable to assume that these measures too will be sensitive to faking given practice with the task. Further, it could be argued that it will be fairly easy to fake responding on word association tasks once it is clear the frequency of alcohol-related responses is the critical score. It is however important to note that indirect measures are notably less sensitive to faking compared to direct measures. To refer to a measure as implicit in the sense that it is goal-independent means that the translation of the to-bemeasured construct into the measurement outcome does not depend on any (theoretically important) goal (De Houwer \& Moors, in press). Whereas affective priming effects have been demonstrated even when participants did not have the goal of evaluating stimuli in the environment (e.g., Spruyt, Hermans, De Houwer, \& Eelen, 2002) and may therefore be goal-independent, it seems unreasonable to refer to the IAT or EAST as goal-independent since the goal of evaluation is critical in both tasks (cf. De Houwer \& Moors, in press). With respect to word association measures, it could be argued that the task is goalindependent in the sense that goals related to alcohol consumption are probably not relevant to the task. However, no studies have examined this issue up to now. Also, a measure can be implicit in the sense that it is purely stimulus-driven, although this is typically very difficult to demonstrate and has not yet been examined for any of the indirect measures described above (De Houwer \& Moors, in press).

A measure can also be implicit in the sense that participants are unconscious of a) the origin of a certain cognitive construct (e.g., Gawronski, Hofmann, \& Wilbur, 2006), b) the stimuli that activate the construct (e.g., De Houwer \& Moors, in press), c) the cognitive construct itself (e.g., De Houwer \& Moors, in press; Fazio \& Olson, 2003; Gawronski et al., 2006), and d) the fact that, or the manner in which, the construct influences behavior, including the performance on an indirect measure (De Houwer, 2006; De Houwer \& Moors, in press; Fazio \& Olson, 2003; Gawronski et al., 2006). Are indirect measures implicit in the sense that participants are unaware of the origins of the cognitive construct that is measured? Olson \& Fazio $(2001,2002)$ have demonstrated that both the IAT and affective priming were able to detect attitudes toward Pokémon characters that were learned through an evaluative conditioning procedure. Hence, these results show that 
indirect measures can register cognitive constructs even though participants may be unaware of the origins of that cognitive construct. It should be noted, however, that participants are also often unaware of the origins of their self-reported cognitions (Gawronski et al., 2006). Second, indirect measures could provide an implicit measure in the sense that participants may be unaware of the stimuli that activate the cognitive construct that is being measured. However, participants typically need to be aware of stimuli that activate the cognitive construct in the IAT, the EAST, priming procedures and word association tasks since they are an integral part of the task, with the only being subliminal priming procedures. Further, whether indirect measures can register unconscious cognitive constructs is almost never checked (cf. Fazio \& Olson, 2003; De Houwer, 2006). Moreover, according to Gawronski et al. (2006) the systematic relationship between indirect and direct measures in fact indicates that participants are typically aware of the cognitive constructs that are measured with both types of tasks. On the other hand, the relationship between indirect and direct measures can be influenced by motivational factors (i.e., social desirability), the degree of cognitive deliberation while selfreporting, and the degree to which direct and indirect measure the same underlying construct (Gawronski et al., 2006). Hence, it could be argued that indirect measures have merit, not because they measure unconscious constructs, but rather because they are less affected by cognitive deliberation, motivational factors. This point will be further discussed in the following section regarding the value of implicit measures. However, at this time, it seems premature to conclude that indirect measures can never be implicit in the sense that they can assess cognitions even though participants are unaware of those cognitions and more research is needed with respect to this issue. Finally, can indirect measures provide implicit measures in the sense that participants may be unaware of the fact that the to-bemeasured cognitions affects the measurement outcome and the way in which the cognitions are translated into the measurement outcome? Up to now, only one study has examined this possibility. Monteith, Voils, and Ashburn-Nardo (2001) found that 64\% of the participants who performed a race IAT were aware of performance differences between the compatible and incompatible combination task. In addition, $37 \%$ of these participants attributed their performance differences to racial attitudes. Thus, although more research is needed, it seems that indirect measures are not per definition implicit in the sense that participants are unaware that a certain cognitive construct affects their performance.

Finally, a measure can be referred to as implicit in the sense that the processes that translate the to-be-measured construct into the measurement outcome are efficient and fast (De Houwer \& Moors, in press). Efficiency is typically examined using a dual-task method. While affective priming effects have been demonstrated to be unaffected by a secondary task (Hermans, Crombez, \& Eelen, 2000), it is unclear to what extent the same can be said about the IAT, the EAST, and word association measures. Further, it could be argued that the indirect measures presented in this overview all rely in some way on reaction times. Hence, these indirect measures all allow limited time for the translation of 
the to-be-measured construct into the measurement outcome. As such it seems reasonable to assume that these tasks all provide implicit measures in the sense that the processes underlying the measurement outcome are relatively fast.

\section{Why Implicit Measures?}

The main benefit of using indirect measures is thus that they may provide implicit measures of cognitions. But why would we be interested in implicit measures? The interest in implicit measures can be easily understood from the perspective of dual process theories. Since the reflective and impulsive system have been characterized by qualitatively different representations, processes, and neural structures, they predict behavior under different circumstances and the differential predictive validity of these two systems is moderated by several factors including one's motivation and opportunity to engage in effortful, controlled processing (e.g., Deutsch \& Strack, 2006; Strack \& Deutsch, 2004). Similarly, the MODE (Motivation and opportunity as DEterminants) model of attitude-to-behavior processes (Fazio, 1990; Fazio \& Towles-Schwen, 1999) proposes that attitudes influence behavior through relatively spontaneous, automatic processes or via more deliberative processes. Importantly, the MODE model proposes that deliberative, controlled processing critically depends on the ability and the motivation to engage in controlled processing. Thus, when implicit measures are defined as measurement outcomes that index cognitive constructs by virtue of processes that can be characterized as automatic, it becomes clear that implicit measures can be particularly valuable as indices of the spontaneous influence of automatically activated cognitions on behavior. Importantly, this perspective on implicit measures also implies that implicit measures will predict behavior under specific circumstances. Specifically, implicit measures will predict behavior that is determined by automatic processes, that is, when the functional properties of the processes underlying the to-be-explained behavior match the functional properties of the implicit measure (De Houwer, 2006). In sum, implicit measures may index automatically activated cognitions that influence behavior in an automatic fashion (cf. De Houwer, 2006; Fazio \& Olson, 2003; Gawronski et al., 2006). For reasons of clarity, the expressions "implicit" or "automatic" will be used for cognitive constructs assessed with indirect measures. Conversely, the terms "explicit" or "controlled" will be used to describe cognitive constructs assessed with direct measures.

\section{FOCUS AND OUTLINE OF THIS THESIS}

This thesis will focus on the IAT as an indirect measure of alcohol-related cognitive processes. In the 8 years since its initial publication, the IAT has received enormous attention and has quickly become one of the most popular indirect measures. The IAT 
owes part of this popularity to its ease of administration as well as to the fact that it produces large and robust effects. Moreover, the IAT has shown satisfactory internal consistency (split-half and Cronbach's alpha), in the region of .80 and higher (e.g., Bosson, Swann, \& Pennebaker, 2000; Cunningham et al., 2001; Greenwald \& Nosek, 2001). Further, the IAT also displays somewhat lower, though acceptable, test-retest reliability with an average test-retest value around .60 (e.g., Bosson et al., 2000; Cunningham, et al., 2001; cf. Greenwald \& Nosek, 2001; Nosek, Greenwald, \& Banaji, 2006). In contrast, lower internal consistencies have been reported for priming measures (e.g., Bosson et al., 2000; Cunningham et al., 2001; Olson \& Fazio, 2003) as well as for the EAST (e.g., De Houwer, 2003; Teige, Schnabel, Banse, \& Asendorpf, 2004). Hence, part of the IATs popularity can be attributed to its achievement of higher reliability compared to other indirect measures such as the EAST and priming measures (cf. Nosek ef al., 2006). Promising results were also found regarding the construct validity of the IAT. First, although most studies have found correlations between the IAT and other indirect measures such as priming measures to be inconsistent, weak, and/or nonexistent (e.g., Bosson et al., 2000; Olson \& Fazio, 2003), Cunningham et al. (2001) demonstrated substantial correlations between the IAT and affective priming measures $(r=.55)$ when controlling for measurement error. Second, a large range of positive correlations between the IAT and direct measures has been found with a low but positive average of .24 (Hofmann, Gawronski, Gschwendner, Le, \& Schmitt, 2005). Moreover, Hofmann et al. (2005) demonstrated that the relationship between the IAT and direct measures increased as a function of both increased spontaneity of self-reports and increased conceptual correspondence between the IAT and direct measures, and varies as a function of methodrelated characteristics of the IAT (e.g., counterbalancing of the two combination tasks in the IAT). Finally, across a wide variety of research domains the IAT has been demonstrated to predict behavior above direct measures (e.g., Asendorpf et al., 2002; Egloff \& Schmukle, 2002; Teachman \& Woody, 2003; see also Poehlman, Uhlmann, Greenwald, \& Banaji, 2005).

Importantly, the reason that the IAT typically achieves higher reliability and validity than other indirect measures such as the EAST and priming measures, could be that the IAT is primarily based on relevant stimulus-response compatibility effects whereas priming measures and the EAST are mainly based on irrelevant stimulus-response compatibility effects (De Houwer, in press). More specifically, IAT effects rely on the compatibility between responses that have become associated with positive or negative valence and the relevant meaning of target stimuli (i.e., the target category to which the stimuli belong) whereas in the EAST and in priming measures, the meaning of stimuli related to the concept of interest is always irrelevant to the task.

Despite these promising results with respect to the IAT as a potential implicit measure of cognitive constructs that influence behavior relatively automatically, results with the IAT in the domain of alcohol use and abuse have been puzzling. Counter intuitively, it was 
demonstrated that evaluative associations with alcohol are negative, regardless of level of alcohol consumption and in clear contrast to participants' self-reported positivity with respect to drinking alcohol (e.g., De Houwer et al., 2004; Wiers et al., 2002, 2005). Moreover, Wiers et al. (2005) demonstrated internal consistencies as well as test-retest reliabilities for the alcohol-IAT in the region of .70, indicating that these results were probably not due to low reliability of the alcohol-IAT. Thus, these results suggest that implicit evaluative associations with alcohol play little to no role in drinking behavior. However, recent research has indicated several limitations of the IAT procedure that may complicate the interpretation of IAT effects. Therefore, the focus of this thesis was to examine whether previous results with the IAT might have been produced by limitations in the IAT procedure and to find ways in which the assessment of alcohol-related cognitive processes with the IAT might be improved in order to further our understanding of the automatic evaluative processes that potentially guide drinking behavior.

\section{Recoding Influences on IAT effects}

IAT effects are assumed to reflect the strength of evaluative associations with the target concepts. However, it has been suggested that compatibility effects in the IAT can also be influenced by recoding processes (Rothermund, Wentura, \& De Houwer, 2005; for evidence see, e.g., De Houwer, Geldof, \& De Bruycker, 2005; Mierke \& Klaver, 2001, 2003; Rothermund \& Wentura, 2001, 2004). Recoding refers to the fact that the target and/or attribute exemplars are categorized on the basis of features that do not match the "nominal definitions" of the respective target and/or attribute concepts (cf. Greenwald, Nosek, Banaji, \& Klaver, 2005). More specifically, in the case of the IAT applied to alcohol research, the nominal features of the target categories are 'alcohol' and 'soda' and target stimuli thus have to be classified based on whether they belong to the alcohol category or the soda category whereas the attribute stimuli need to be classified based on valence. Importantly, it makes a difference for the interpretation of a given IAT effect whether target and attribute exemplars were classified based on their nominal features and compatibility effects are therefore based on evaluative associations with the target concepts, or whether target and attribute stimuli were classified based on other features including familiarity, valence, salience or any other information that can facilitate IAT performance in one combination task by reducing the two-dimensional classification task (i.e., target classification and attribute classification) to a one-dimensional classification task (e.g., all stimuli are classified based on familiarity) but not in the other combination task. Influences of recoding on IAT effects are mainly problematic because they tend to obscure the nature of the processes that underlie compatibility effects in the IAT. It is often not clear whether recoding of the categorization task has actually occurred, and if so, what kind of recoding has taken place. It is therefore difficult to interpret the meaning of 
compatibility effects in the IAT because it is unclear with regard to which feature the target and attribute categories are compatible.

In the first part of this thesis, it was examined to what extent the alcohol-IAT could reflect recoding influences rather than evaluative associations with the concept alcohol. One influential recoding account of the IAT in terms of salience asymmetries was proposed by Rothermund and Wentura $(2001,2004)$. More specifically, they proposed that salience or figure-ground asymmetries can cause IAT-effects, independent of implicit associations. When one of the categories of an IAT dimension is more salient than the other, the most salient category will attract attention. This salient category becomes the so-called figure, whereas the other category constitutes the (back)ground. Subsequently, when both the target and attribute figure are mapped onto the same response, performance will be better compared to when one figure and one ground category are assigned to the same response. Rothermund and Wentura $(2001,2004)$, in addition, argue that negative and unfamiliar stimuli are typically more salient than positive and familiar stimuli. Hence, in the alcohol-IAT, negative attributes were probably more salient than positive attributes. Further, it could be argued that alcohol stimuli are potentially more salient than soda stimuli for heavy drinkers (cf. attentional bias; Field, Mogg, \& Bradley, 2006). Thus, the finding that alcohol (relative to soda) was more easily paired with negative stimuli than with positive stimuli in the IAT (e.g., Wiers et al., 2002) could be due to salience asymmetries causing faster performance for the consistent mapping of the salient negative and alcohol categories versus the non-salient positive and soda categories than for the reversed stimulus-response mapping. In chapter 2, it was examined whether reversing salience asymmetries between the alcohol and soda target categories by manipulating the familiarity of the target exemplars would also reverse the IAT effect. Further, in chapter 3, a newly introduced variant of the IAT, the IAT-RF (short for "IAT-Recoding Free"; Rothermund, Teige-Mocigemba, \& Wentura, 2006) was used to measure alcohol-related evaluative associations. The IAT-RF aims to eliminate effects of recoding by abandoning the block structure of the standard IAT. In the IAT, compatible and incompatible trials are presented in separate blocks of the task. This structure creates an extended consistent mapping of categories onto responses in the compatible block, which may invite recoding processes. In the IAT-RF, on the other hand, compatible and incompatible response assignments of categories vary randomly between trials within a single block. This random switching of response assignments undermines any type of recoding, because efficient recoding requires a consistent mapping of categories onto responses (Roßnagel, 2001; Shiffrin \& Schneider, 1977; Strayer \& Kramer, 1994).

It is important to note that, even when recoding processes that are based on features other than the nominal features of the categories are eliminated in the IAT, participants could still re-construe the categories in the IAT in a way that is inconsistent with the nominal features of the categories as intended by researcher. Research has demonstrated that IAT effects arise mainly because the two combination tasks differ with respect to 
relevant stimulus-response compatibility (De Houwer, 2001; see also De Houwer, 2003, in press). This means that IAT effects depend on the properties (e.g., valence) of the categories rather than the properties of the individual exemplars (i.e., irrelevant stimulusresponse compatibility). Specifically, the relevant feature to which participants need to react is the category to which a stimulus belongs. During compatible trials, the relevant feature of the stimulus and the to-be-emitted response will have the same valence (e.g., flowers and positive vs. insects and negative) due to the intrinsic meaning of the category concepts and the extrinsic meaning of the responses. During incompatible trials, in contrast, the relevant stimulus feature and the responses are less compatible because the extrinsic meaning of the responses will be ambiguous (e.g., flowers and negative vs. insects and positive).

However, even though IAT effects are primarily driven by properties of the categories, properties of the individual exemplars can still influence IAT performance (e.g., Govan \& Williams, 2004; Mitchell, Nosek, \& Banaji, 2003), probably because they affect how the categories are conceptualized (De Houwer, in press; Govan \& Williams, 2004). One way in which exemplars can influence IAT performance is for instance by promoting a conceptualization of the evaluative attribute categories as normatively positive or negative It was suggested that IAT effects might reflect extrapersonal evaluative associations with the target concepts (e.g., normative cultural knowledge with respect to the target concepts) rather than personal evaluative associations that are relevant to behavior (Olson \& Fazio, 2004; see also Karpinski \& Hilton, 2001). Olson and Fazio (2004) argue that the accessibility of normative information in the IAT is increased because the category labels "positive" and "negative" carry a normative implication which is further underscored due to the category exemplars which are typically normatively positive or negative, and because giving error feedback also suggests that there is a normatively correct response.

Therefore, Olson and Fazio (2004) introduced the personalized IAT that may reduce extrapersonal contamination through three modifications of the IAT procedure. First, the personalized IAT eliminates normative implications associated with the labels 'positive' and 'negative' through the use of the labels 'I like' and 'I dislike'. Second, it uses attribute stimuli that are not normatively associated with valence but that have little evaluative consensus (large variability) while still being attitude-evoking (e.g., football, coffee). Finally, no error feedback is given to participants to avoid suggesting that there is a normative correct response. The personalized IAT was demonstrated to reveal less racial prejudice and was more strongly correlated with direct measures, suggesting a reduced influence of extra-personal associations (Olson \& Fazio, 2004). Nosek and Hansen (2007), in contrast, argue that the original and the personalized IAT both capture unique attitude-relevant aspects, reflecting the multidimensionality of attitudes. In a series of studies, evidence for the multidimensionality of attitudes was found and results suggested that both IAT versions were capturing different aspects of attitude constructs. Since negative evaluative associations could reflect negative extrapersonal (culturally shared) 
associative information that is irrelevant to behavior, it was examined in chapter 4 whether the personalized IAT would also reveal evidence for negative evaluative associations with alcohol similar to the IAT. It is, however, important to note that the IAT presents stimulus words for which there is no evaluative consensus, but that are nevertheless the same for all participants. It is questionable how well an IAT that presents standard stimuli can capture participants' personal associations, since standard stimuli will probably differ in the degree to which they are both known and liked across participants (Fadardi, Cox, \& Klinger, 2006). This is particularly true when applied to alcohol research since there is probably great variation in the alcoholic drinks that are known and liked by participants. Therefore, a personalized IAT was tested in chapter 5 that presented stimuli that were selected by participants as evaluatively positive or negative as well as participants own favorite alcoholic drinks and soft drinks.

\section{Relative Nature of the IAT Category Dimensions}

It is important to note that the standard IAT procedure measures the strength of two target concepts with two evaluative categories, positive and negative. Consequently, IAT effects can only indicate whether the target concepts are more associated with one of the evaluative categories relative to the other evaluative category. As such, this bipolarity of the evaluative dimension potentially precludes the assessment of evaluative associations that determine drinking behavior given that ambivalence is typically quite strong in the case of addictive behaviors (Conner \& Sparks, 2002) and even at the core of some of its definitions (e.g., Orford, 2001). Moreover, from the perspective of dual process models, it is possible that both positive and negative evaluative associations exist simultaneously in the impulsive system (e.g., Deutsch \& Strack, 2006). Thus, previous findings with the IAT suggest negative evaluative associations with alcohol are stronger than positive evaluative associations with alcohol. However, due to the bipolarity of the attribute dimension in the IAT, it is theoretically possible that someone who possesses only strong negative evaluative alcohol associations but virtually no positive evaluative alcohol associations and someone who has strong evaluative positive alcohol associations and even stronger negative evaluative alcohol associations yield IAT scores of similar magnitude. Hence, valuable information is lost when evaluative associations with target concepts that are potentially characterized by ambivalence such alcohol are assessed in a bipolar format. Therefore, in the second part of this thesis, a unipolar variant of the IAT (Jajodia \& Earleywine, 2003) was used to simultaneously assess both positive evaluative and negative evaluative alcohol-related associations. Specifically, the unipolar IAT enables the simultaneous assessment of both positive and negative evaluative associations with alcohol by contrasting both positive and negative attributes with attribute categories made up of unrelated neutral words (e.g., basic, intermediate). 
Importantly, the IAT is also bipolar with respect to the target concepts. Consequently, the IAT cannot reveal evaluative associations with single target concepts such as alcohol, but is instead limited to measuring the relative strengths of pairs of evaluative associations. While this relative nature of the IAT is not a problem for most research questions as many attitude concepts have a complementary category that can be used as a contrast in the IAT (e.g., black vs. white, old vs. young), it is a problem when one is interested in implicit associations with a single target concept or when the target concept of interest does not have a natural complement as in the case of addictive behaviors. Importantly, in the case of addictive behaviors, the contrast category for a certain addictive substance can in fact be anything else that is not related to the substance (e.g., smoking versus not smoking). While contrasting the concept alcohol with a concept related to soft drinks such as soda might have a natural feel to it (although there are plenty other drinks available besides alcoholic drinks and soft drinks), we are nevertheless only interested in assessing individual differences with respect to alcohol-related evaluative associations and not individual differences regarding evaluative associations with soda. Hence, the relative nature of the IAT obscures the interpretation of previous findings with the IAT since IAT scores can reflect both evaluative associations with alcohol, evaluative associations with soda, or a combination of both. Possibly, positive evaluative associations with soda were responsible previously reported IAT effects and not, or to a lesser extent, negative evaluative associations with alcohol. Also, it should be noted that the soda contrast might have caused a context effect in such a way that evaluative associations with alcohol could be generally positive (depending on level of alcohol consumption), but rather negative when contrasted with soft drinks, which could be a somewhat healthier alternative to drinking alcohol.

In chapter 6, alcohol-related associations were assessed with unipolar IATs in order to simultaneously address both positive and negative evaluative associations as well as both arousal and sedation associations (for example as in Wiers et al., 2002). In addition, alcohol-related associations were assessed relative to soda as well as relative to a different contrast category made up of neutrally evaluated animals in order to examine the influence of the contrast category soda that is typically used in the alcohol-IAT. Finally, associations with the concept 'alcohol' could be affected by negative cultural connotations (cf. Karpinski \& Hilton, 2001) or might even be behavior-irrelevant since the alcohol consumption of the participants included in the studies reported here (i.e., general student population) was probably almost entirely confined to drinking beer (cf. extrapersonal associations; chapter 3 and chapter 4). Therefore, it was also examined whether unipolar IATs that assessed associations with the target concept 'alcohol' and unipolar IATs that measured associations with the target concept 'beer' would yield similar results. Since salience asymmetries might be an important contributing factor to effects in the unipolar IAT (possibly, the 'neutral' attribute categories were always less salient than the positive, negative, arousal, and sedation categories), salience asymmetries between the target and 
attribute categories used in the unipolar IATs were objectively assessed with visual search tasks in order to explore whether salience asymmetries might have been responsible for observed IAT effects.

While comparing IAT results with different contrast categories as in chapter 6 can be useful, using the IAT to assess implicit associations with single target concepts that are not part of a natural dichotomy remains problematic. In chapter 7 and 8, this issue was further addressed by applying a non-relative indirect measure to the study of alcohol-related associations: the Single-Target IAT (ST-IAT; Wigboldus, Holland \& van Knippenberg, 2006). Wigboldus et al. (2006) developed the Single Target IAT (ST-IAT) which is structurally similar to the IAT with the difference that only one target category is used. Participants are instructed to classify both this target and one attribute category (e.g., alcohol and negative) with one response key and the other attribute category (e.g., positive) with the second response key. Unlike the IAT, the ST-IAT measures associations between only one target concept and the attributes without a second contrasting target category. Similarly, Karpinski and Steinman (2006) introduced the Single Category Implicit Association Test (SC-IAT) which is conceptually similar to the ST-IAT, differing only in minor procedural details. Karpinski and Steinman provided initial support for the reliability and validity of the SC-IAT and demonstrated that the SC-IAT, like the ST-IAT, can yield additional information that is not reflected in relative IAT scores. In chapter 7 and 8 , unipolar ST-IATs were used to study alcohol-related evaluative associations. Importantly, by presenting the ST-IAT in a unipolar format, similar to what was done for the IAT (cf. Jajodia \& Earleywine, 2003; chapter 6), the bipolarity of both the target and the attribute dimension was eliminated. Further, in chapter 7, evaluative associations were assessed with either the concept 'alcohol' or the concept 'beer' as in chapter 6. In chapter 8, unipolar IATs that assessed evaluative associations with alcohol relative to beer were directly compared with unipolar ST-IATs that measured single evaluative associations with alcohol. Moreover, single evaluative associations with soda were also measured with unipolar ST-IATs in order to examine the extent to which single evaluative alcohol and soda associations contribute to relative IAT scores. 



$$
\text { D) }
$$




\section{PART | \\ RECODING INFLUENCES ON PERFORMANCE IN THE ALCOHOL-IAT}




$$
\text { D) }
$$




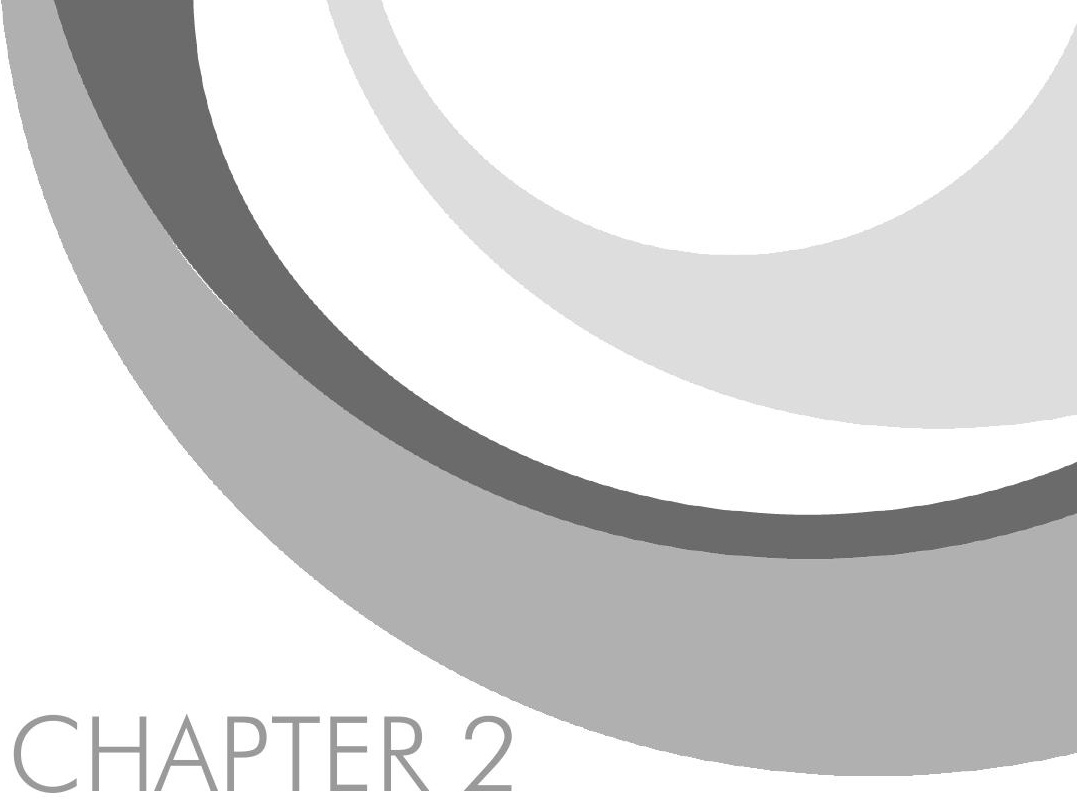

\section{Testing the Salience Asymmetry Account of the Alcohol-IAT}

Published as: Houben, K., \& Wiers, R. W. (2006). A Test of the Salience Asymmetry Interpretation of the Alcohol-IAT. Experimental Psychology, 53, 292-300. 


\begin{abstract}
K. Rothermund and D. Wentura (2004) showed how Figure-Ground (FG) asymmetries produce effects on the Implicit Association Task (IAT), independent of associations. Here, the FG account was tested for the robust finding that drinkers show a negative alcohol-IAT effect while being positive on explicit measures. FG asymmetries were manipulated through familiarity of alcohol-IAT target categories and assessed with visual search tasks. Supporting the FG account, the familiarity manipulation influenced the IAT effect in the expected direction and the IAT effect correlated with FG asymmetries. Contrary to the FG account, however, the IAT effect was not reversed, and IAT effects were predicted by alcohol use but not by FG asymmetries. Hence, the FG account only partly explains the negative alcohol-IAT effect.
\end{abstract}

\title{
INTRODUCTION
}

In the seven years since its initial publication, the Implicit Association Test (IAT; Greenwald, McGhee, \& Schwartz, 1998) has become a widely applied instrument to indirectly assess associations. The IAT is a speeded classification task in which words are classified into two times two categories: Two target categories (e.g., alcohol vs. sodas) and two attribute categories (e.g., positive vs. negative). In the critical IAT phases, the categories are assigned to two response keys in two different combinations (e.g., alcohol-negative vs. soda-positive and alcohol-positive vs. soda-negative). The IAT effect is the performance difference between these combinations and the underlying assumption is that it is easier to combine concepts that are associated in memory than to combine concepts that are not or weakly associated (Greenwald et al., 1998). The IAT owes its popularity to its flexibility, large effect sizes and good reliability (e.g., Bosson, Swann, \& Pennebaker, 2000; Cunningham, Preacher, \& Banaji, 2001; Nosek, Greenwald, \& Banaji, 2006). Promising results were also found for its construct validity, with small but positive correlations between the IAT and corresponding self-report measures (see Hofmann, Gawronski, Gschwendner, Le, \& Schmitt, 2005) and good predictive validity (e.g., Poehlman, Uhlmann, Greenwald, \& Banaji, 2005).

Despite its popularity, many issues surrounding the IAT remain unresolved, including the interpretation of IAT effects. Whereas Greenwald et al. (1998) argue that IAT effects reflect the strength of implicit associations in memory, Rothermund and Wentura (2004) have proposed a non-associative account of IAT effects, based on salience asymmetries. In a series of studies, they convincingly demonstrated that salience or "Figure-Ground" (FG hereafter) asymmetries can produce IAT effects independent of underlying associations. 
When categories forming a dimension in the IAT differ in salience, the salient category will act as figure against the less salient background category. Consequently, Rothermund and Wentura (2004) argue that performing the IAT should be easier for consistent mappings of the figure categories of both IAT dimensions than when one figure and one ground category are assigned to one response. Furthermore, Rothermund and Wentura (2004) showed that visual search tasks can be used to objectively assess FG asymmetries within IAT dimensions and that controlling for FG asymmetries can render the predictive validity of the IAT nonsignificant.

In a comment on Rothermund and Wentura (2004), Greenwald, Nosek, Banaji, and Klaver (2005) argue that Rothermund and Wentura (2004) based their evidence for the FG interpretation of the IAT upon non-standard procedures that diminish the validity of the IAT. Specifically, it is argued that Rothermund and Wentura (2004) have used artificial non-categories that are not representative of most IAT studies and that they used the conventional millisecond (ms) measure of IAT effects instead of the improved new Dmeasure (Greenwald, Nosek, \& Banaii, 2003). Greenwald et al. (2005) conducted two experiments using IATs that were closely modeled after the ones used by Greenwald et al. (1998), to further examine the validity of the FG asymmetry theory. Also, salience asymmetries were assessed with three measures modeled after the visual search task used by Rothermund and Wentura (2004). Inconsistent with the FG hypothesis, the results did not show faster performance for consistent mappings of salient categories or correlations between the IAT and salience asymmetry measures. Greenwald et al. (2005) conclude that FG asymmetries are neither necessary nor sufficient to produce IAT effects.

In response to Greenwald et al. (2005), Rothermund, Wentura, and De Houwer (2005) state that using non-standard IAT procedures is in fact necessary to examine how the IAT functions in standard cases and to experimentally dissociate the influence from FG effects and associations which are typically confounded in standard IAT procedures. In addition, Rothermund et al. (2005) argue that reanalysis of the data reported by Rothermund and Wentura (2004) with the D-measure yielded the same pattern of results as the analysis with the conventional ms measure. Rothermund et al. (2005) also state that the null findings reported by Greenwald et al. (2005) are probably due to the task irrelevance of their salience manipulations and to the use of visual search tasks that differed from the one developed by Rothermund and Wentura (2004).

\section{THE PRESENT STUDY}

The present study tested the FG interpretation for a puzzling, yet robust IAT finding in alcohol research. Several alcohol-IAT studies have demonstrated that participants find it easier to combine alcohol and negative words in the IAT than to combine alcohol and positive words (De Houwer, Crombez, Koster, \& De Beul, 2004; Wiers, van de 
Luitgaarden, van den Wildenberg, \& Smulders, 2005; Wiers, van Woerden, Smulders, \& de Jong, 2002). Interestingly, the same participants indicated that they expected positive effects from drinking alcohol on explicit expectancy measures (Wiers et al., 2002). This negative alcohol-IAT effect could be meaningful and representative of, for example, early negative experiences with alcohol (Rudman, 2004) or problems related to alcohol use (Wiers, Houben, Smulders, Conrod, \& Jones, 2006). Alternatively, this IAT effect could be an artifact of FG asymmetries in the IAT. According to Rothermund and Wentura (2004), negative stimuli generally act as figures against a positive background. Consequently, if alcohol is more salient than soda (which often forms the contrast target category in the alcohol-IAT), FG theory predicts faster performance for the combined mapping of alcohol and negative words than for the combination of alcohol and positive words, irrespective of meaningful associations.

This FG account of the alcohol-IAT was tested in two ways. First, two positive-negative IAT versions were designed in which salience asymmetries between the alcohol and soda target categories were directly manipulated through familiarity of the category exemplars. The familiar alcohol IAT contrasted familiar alcoholic drinks with unfamiliar sodas, while the unfamiliar alcohol IAT presented unfamiliar alcoholic drinks vs. familiar sodas. This manipulation should produce a salience asymmetry with the unfamiliar categories being more salient than the familiar categories (Rothermund \& Wentura, 2004). Accordingly, FG theory predicts faster performance for the combination of alcohol with negative words compared to the combination of alcohol with positive words in the unfamiliar alcohol IAT. However, in the familiar alcohol IAT, the FG account predicts a reversal of this IAT effect with faster performance for the combination of alcohol with positive words relative to the combination of alcohol with negative words. If, however, the negative alcohol-IAT effect reflects a meaningful association, the salience manipulation should not cause such a reversal of the IAT effect and performance should be faster for the combination of alcohol with negative than for the combination of alcohol and positive in both IAT versions.

Second, visual search tasks (identical to the one used by Rothermund \& Wentura, 2004) were used to independently assess salience asymmetries within IAT dimensions. Negative words were expected to act as figure against a positive background in both IATs while the alcohol and soda categories were expected to be figure only when they consisted of unfamiliar exemplars. The FG account predicts an influence of the central familiarity manipulation on IAT effects and expects a correlation between IAT effects and FG asymmetries. In addition, according to the FG account, FG asymmetries should predict IAT effects above self-reported alcohol use and alcohol-related problems and they should attenuate or eliminate the relationship between self-reported behavior and the IAT. The associative interpretation of the IAT predicts the same IAT effect in both familiarity conditions and does not expect an attenuation of the relationship between alcohol use and IAT effects by FG asymmetries. 


\section{METHOD}

\section{Participants}

Forty-six students of Maastricht University $(11$ men, 35 women; mean age $=21.6$ years, $S D=2.25)$ participated in the study for course credit. On the Alcohol Use Disorder Identification Test (AUDIT; Saunders, Aasland, Babor, De la Fuente, \& Grant, 1993), participants' mean score was 9 (SD $=4.54)$ which is comparable to American college students (Fleming, Barry, \& MacDonald, 1991). About $60 \%$ of the participants scored 8 or higher on the AUDIT, and about 30\% scored 11 or higher, which are the respective cut-off scores for being a hazardous drinker and for the screening of alcohol problems (Saunders et al., 1993). On average, participants drank alcohol about once a week, with 3 to 4 alcoholic drinks on each occasion and had a binge almost once a month.

\section{Materials and Measures}

Alcohol use and problems. Alcohol use and alcohol-related problems were assessed with the AUDIT (Saunders et al., 1993). The AUDIT consisted of 10 questions in a multiple choice format. The first three questions related to alcohol use $(\alpha=.80)$, the other seven to alcohol-related problems $(\alpha=.83)$.

Implicit Association Task. The selection of the IAT alcohol and soda targets was based on a pilot study with the same participants approximately a week before the test. A number of alcoholic drinks and sodas were rated on familiarity on a 7-point Likert scale $(1=$ never seen/heard about, $7=$ very often seen/heard about). The five most familiar and the five most unfamiliar alcoholic drinks $(M=5.31, S D=1.17$ and $M=2.34, S D=.76$, respectively) were selected for the unfamiliar and familiar alcohol categories. The same procedure was followed for the soda categories (familiar sodas: $M=5.85, S D=.87$; unfamiliar sodas: $M=2.06, S D=.73)$. Evaluative ratings, obtained from a subsample of the participants in the present study $(\mathrm{N}=17)$, showed that both unfamiliar alcohol and unfamiliar soda stimuli were evaluated as neutral $M=3.61, S D=.67$, and $M=4.06, S D$ $=.88$, respectively). Familiar alcohol stimuli were also evaluated as neutral $(M=4.41, S D$ $=.99$ ) while familiar soda stimuli were more positively evaluated $(M=5.28, S D=1.02)$. Paired samples t-tests confirmed that there was no significant difference in valence between the familiar alcohol and the unfamiliar soda category, $t(16)=1.24, p=.235$, while the familiar soda category was evaluated as significantly more positive than the unfamiliar alcohol category, $t(16)=-5.507, p<.001$. Valence words were identical to those used by Wiers et al. (2002). All stimuli are presented in the Appendix.

Participants performed one of two IAT versions: The IAT with familiar alcohol vs. unfamiliar soda or the IAT with unfamiliar alcohol vs. familiar soda. Both IATs were programmed in ERTS 3.18 (Beringer, 1996), modeled after the IAT by Greenwald et al. 
(1998). Both IAT versions consisted of five blocks. Participants first received 20 trials of target discrimination practice in which all targets were presented twice. Next, they performed 20 trials of attribute classification practice. The third block was the combination block during which both target and attribute stimuli were presented for 40 trials. Next, participants practiced the reversed attribute discrimination, followed by the reversed combination block. The intertrialinterval was 250 milliseconds. Feedback ('wrong', 'too fast' [ $300 \mathrm{~ms}$ ] and 'too slow' [> $3000 \mathrm{~ms}$ ]) was presented. Internal consistencies, calculated as in Greenwald et al. (2003), were .84 for the familiar alcohol IAT, and .87 for the unfamiliar alcohol IAT.

Visual Search Task. Participants performed two visual search tasks: One visual search task assessed salience asymmetries between the alcohol and soda categories and the other between the valence categories. Participants performed the visual search tasks with the same stimuli as in the IAT. Visual search tasks were programmed in ERTS 3.18 (Beringer, 1996), exactly following Rothermund and Wentura (2004). First, participants received 20 categorization trials to practice the assignment of stimuli to their respective categories. Immediately after the practice block, the corresponding visual search task was performed, consisting of 12 practice trials and 64 experimental trials. During each trial, four stimuli were presented. On half of the trials, all stimuli belonged to the same category ("same" trials), whereas in the other half, three stimuli belonged to one category and the fourth stimulus belonged to the other category ("different" trials). Participants were instructed to indicate with two response keys whether all stimuli on screen belonged to the same category or whether one of the stimuli belonged to a different category. On half of the trials, the majority of the stimuli (three or four out of four) belonged to the first category of the respective dimension, on the other half, the majority of the stimuli belonged to the second category. Stimuli were presented in the corners of either a virtual square or a virtual diamond. During different trials, the stimulus that differed appeared twice at each of the four possible locations. Each trial started with a ready signal $\left({ }^{\prime} X^{\prime}\right)$ in the middle of the screen, which was replaced by a cue $\left({ }^{* \prime}\right)$ when participants pressed the space bar. After an interval of $500 \mathrm{~ms}$, the stimuli were presented around the cue in black against a grey background. Response labels (same or different) were presented in the upper right and left corner of the computer screen. Stimulus words remained on screen until the correct response was given. Feedback ('ERROR—press correct key and continue') was presented until the correct response was given.

Alcohol Expectancy Questionnaire.. The alcohol expectancy questionnaire consisted of 10 items of which 5 items referred to positive expectancies $(\alpha=.87)$ and 5 items represented negative expectancies $(\alpha=.84)$. Positive and negative expectancies were negatively correlated, $r(46)=-.39, p=.008$. Each item asked participants to indicate on a Visual Analogue Scale (VAS) how much they agreed or disagreed with the following statement: "After drinking alcohol, I feel ..." which was completed with the same positive and negative words as presented during the IAT. 


\section{Design and Procedure}

After signing the informed consent form, participants randomly performed the familiar alcohol IAT or the unfamiliar alcohol IAT. The assignment of the alcohol and sodas target categories to the response keys was balanced across participants. Half of the participants received the alcohol-negative combination (compatible response assignment) first and then the alcohol-positive combination (incompatible response assignment) (CR condition), and half received the reversed order of combination tasks (RC condition; see Greenwald et al., 1998). Next, participants performed the two visual search tasks. The response assignment of same and different trials was balanced across participants as well as the order of the visual search tasks. Participants then filled out the alcohol expectancy questionnaire and the AUDIT.

\section{RESULTS}

\section{Implicit Association Task}

IAT effects were calculated with both the conventional measure in ms (Greenwald et al., 1998) and the new D600 measure (Greenwald et al., 2003)' . First, for each participant, mean response latencies were calculated separately for the compatible and incompatible response assignments. Next, the conventional ms measure of IAT effects was calculated as the difference between these two means in such a way that higher IAT scores indicated faster performance for the compatible (alcohol + negative vs. soda + positive) than for the incompatible (alcohol + positive vs. soda + negative) combination task. The D600 measure of IAT effects was calculated in the same direction as the conventional ms IAT measure. Further, following the formula presented by Greenwald et al. (2003), practice blocks were now included, error penalties $(600 \mathrm{~ms})$ were given in case of a wrong response, and results were standardized at the level of the participant.

Both IAT effect measures were entered in a 2 (familiarity) $\times 2$ (order of combination tasks: CR or RC) univariate analysis of variance (ANOVA). Results for the D600 measure showed a significant order effect, $F(1,42)=6.35, p=.016, \eta=.13$, but no significant effect of familiarity, $F(1,42)=1.90, p=.176$, in the absence of a significant interaction $(p>.80)$. For the conventional ms measure, results showed both a significant effect of familiarity, $F(1,42)=4.81, p=.034, \eta=.10$, and of order, $F(1,42)=11.29, p=$

\footnotetext{
'We analyzed both the conventional scoring algorithm (ms) and the new D600 algorithm and report results with both measures of IAT effects. There are three reasons for doing this: First, the new D600 measure had been found to yield different results than the conventional ms measure (e.g., Wiers et al., 2005). Second, there is no consensus regarding the optimal measure for IAT effects (see Greenwald et al., 2005 vs. Rothermund et al., 2005). Third, using the ms measure enables comparison with earlier IAT research and methods.
} 
$.002, \eta=.21$, in the absence of an interaction effect $(p>.80)$. Next, IAT effects were analyzed separately for both orders of combination tasks and separately for both familiarity conditions with one-sample t-tests. Mean response latencies for compatible and incompatible combination tasks and mean IAT effects are presented per combination task order and per familiarity version in Table 1. In the CR condition, both the D600 and the ms measure of the IAT effect were highly significant, $t(20)=5.31, p<.001, d=1.16$ and $t(20)=5.23, p<.001, d=1.14$, respectively, indicating that performance was faster for the combination of alcohol and negative than for the combination of alcohol and positive. In the RC condition, this IAT effect was smaller, but still significant for the conventional ms measure, $t(24)=2.22, p=.036, d=.45$, while borderline significant for the D600 measure, $t(24)=1.82, p=.081, d=.36$. Next, IAT effects were analyzed per familiarity condition. Results showed that IAT effects measured with both the D600 and the conventional ms measure were significant in the unfamiliar alcohol IAT condition, $t(21)$ $=4.08, p=.001, d=.87$ and $t(21)=5.20, p<.001, d=1.11$, respectively, and were smaller but still significant in the familiar alcohol IAT condition, $t(23)=2.46, p=$ $.022, d=.50$, and $t(23)=2.27, p=.033, d=.46$, respectively. These results indicate that, in both familiarity conditions, performance was faster when alcohol and negative were mapped onto the same response than when alcohol and positive shared a response. In sum, results with both IAT scoring algorithms indicate that performance was faster for the combination of alcohol and negative words compared with the combination of alcohol and positive words, in both combination task order conditions and in both familiarity conditions.

Table 1

Mean response latencies in compatible and incompatible combination tasks and mean IAT effects (standard deviations are shown in parentheses).

\begin{tabular}{|c|c|c|c|c|c|}
\hline \multirow[t]{2}{*}{ Variable } & & \multicolumn{2}{|c|}{ Combination Task } & \multicolumn{2}{|c|}{ IAT effect } \\
\hline & & Compatible & Incompatible & $\begin{array}{c}\text { Conventional ms } \\
\text { measure }\end{array}$ & $\begin{array}{c}\text { D600 } \\
\text { measure }\end{array}$ \\
\hline \multirow[t]{2}{*}{ Familiarity } & Familiar alcohol & 708.30 (153.21) & $819.39(211.00)$ & 111.09 (240.29) & $.47(.94)$ \\
\hline & Unfamiliar alcohol & 637.81 (90.12) & $886.50(234.69)$ & $248.69(224.54)$ & $.83(.96)$ \\
\hline \multirow[t]{2}{*}{ Order } & $C R$ & $689.06(124.76)$ & $980.34(240.28)$ & $291.28(255.48)$ & $1.01(.87)$ \\
\hline & $\mathrm{RC}$ & $662.43(136.64)$ & $743.25(134.80)$ & 80.82 (181.79) & $.34(.93)$ \\
\hline
\end{tabular}

Note. Mean response latencies and mean IAT effects are shown separately for both familiarity conditions (familiar alcohol and unfamiliar alcohol) and both combination task orders (CR and RC). CR = compatible combination task first, $R C=$ incompatible combination task first. In the compatible combination task, alcohol and negative words were assigned to the same response while alcohol and positive words shared a response in the incompatible combination task. The conventional IAT effect measure in ms was computed as the difference between the means for the compatible and incompatible combination tasks. The D600 IAT effect measure was calculated by dividing this difference score by the standard deviation of all response latencies in both combination tasks. 


\section{Salience Asymmetries}

Following Rothermund and Wentura (2004), trials with erroneous responses were left out of the analyses. For each visual search task, mean response latencies were computed for trials on which the majority of the stimuli (distractors) belonged to the first or second category of the respective dimension (e.g., alcohol or soda). This was done separately for same and different trials because same trials have been found to be a more sensitive indicator of FG asymmetries (Rothermund and Wentura, 2004, footnote 5). Furthermore, mean response latencies were log-transformed before analyses.
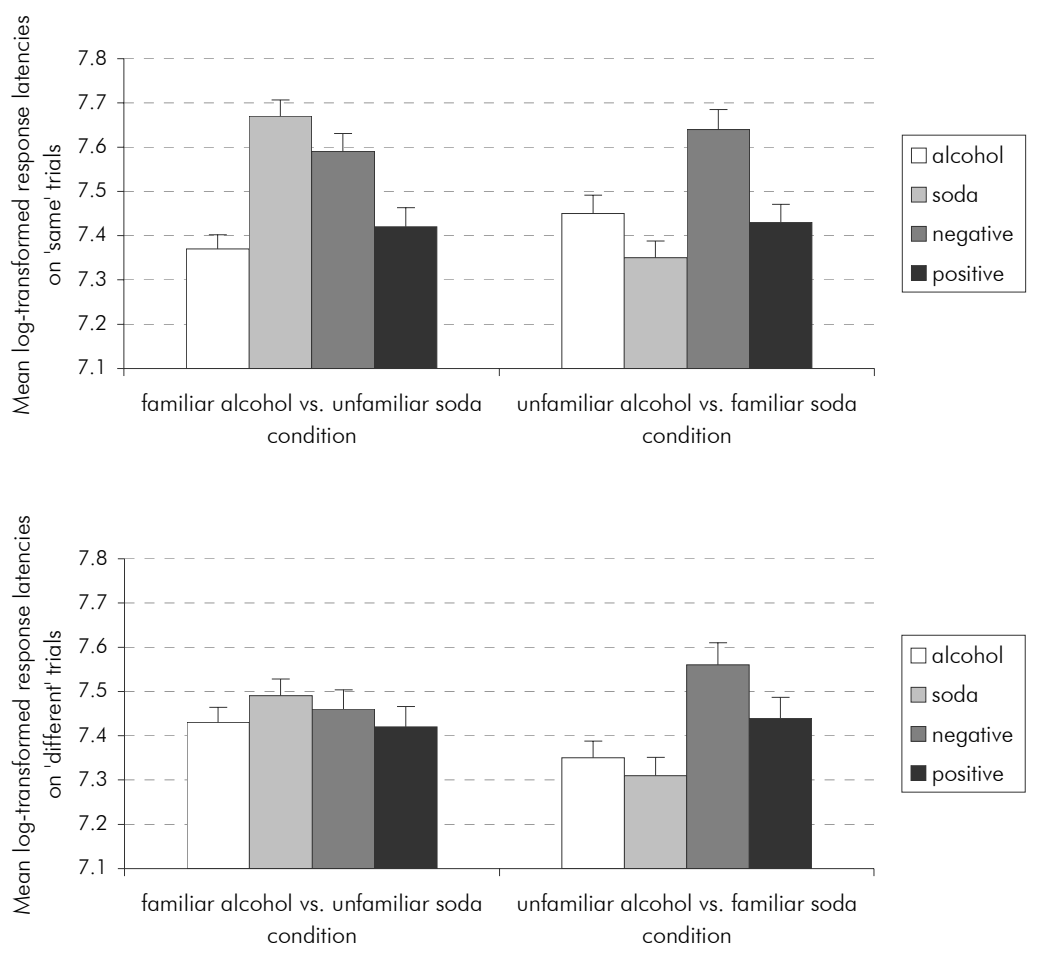

Figure 1. Log-transformed mean response latencies for each category presented in the visual search task are shown separately for the two familiarity conditions. Visual search tasks either examined salience asymmetries between the alcohol and soda target categories or between the positive and negative attribute categories. Log-transformed response latencies are also shown separately for same (top) en different (bottom) search trials. On same trails, participants responded to four stimuli that all belonged to the same category (e.g., four alcohol stimuli during an alcohol same trial), while on different trials, participants responded to three distractors from one category and one target from the other category (e.g., three alcohol stimuli and one soda stimulus during an alcohol different trial). Longer response latencies for a category compared to the other indicate that this category was more salient than the other category. 
As can be seen in Figure 1, responding was significantly slower for trials with unfamiliar soda distractors than for trials with familiar alcohol distractors, $t(23)=-10.11, p<.001$, $d=2.06$, and $t(23)=-3.17, p=.004, d=.65$, for same and different trials, respectively. Responding was also significantly slower for same trials with unfamiliar alcohol distractors compared with same trials with familiar soda distractors, $t(21)=2.57$, $p=.018, d=.55$. However, no salience asymmetry between unfamiliar alcohol and familiar soda was found for the different trials, $t(21)=1.62, p=.121$. Further, in the familiar alcohol condition, results demonstrated significantly slower responses for same trials with negative distractors compared to same trials with positive distractors, $t(23)=$ $8.48, p<.001, d=1.73$, while this salience asymmetry was borderline significant for different trials, $t(23)=1.98, p=.059$. Similarly, in the unfamiliar alcohol condition, processing negative words was significantly slower than processing positive words during both same trials, $t(21)=7.94, p<.001, d=1.69$, and different trials, $t(21)=6.03, p$ $<.001, d=1.29$. Hence, consistent with the hypotheses, the unfamiliar alcohol and soda target categories were more salient than the familiar alcohol and soda target categories while the negative attribute category was more salient than the positive attribute category.

Before entering FG asymmetries in correlational analyses, difference scores were computed separately for same and different trials, by subtracting mean log-transformed response latencies for trials with soda and positive distractors from mean log-transformed response latencies for trials with alcohol and negative distractors, respectively. Therefore, positive difference scores indicated that alcohol and negative stimuli were more salient than soda and positive stimuli, respectively. To test whether the IAT was confounded with these FG asymmetries, partial correlations, controlled for order of IAT combination tasks, were computed between the IAT and FG asymmetries. Partial correlations between the IAT and FG asymmetries for the whole sample as well as per familiarity condition are shown in Table 2. Overall, a significant correlation was found between the conventional ms measure of IAT effects and salience asymmetries between alcohol and soda on same trials. When partial correlations were computed per familiarity condition, this correlation was more pronounced for the familiar alcohol condition than for the unfamiliar alcohol condition, but it did not reach statistical significance in either familiarity condition. No significant correlations emerged between salience asymmetries and the D600 measure of IAT effects. 
Table 2

Partial correlations between the IAT and expectancies, alcohol use and alcohol-related problems, and salience asymmetries, controlled for order of IAT combination tasks.

\begin{tabular}{|c|c|c|c|c|c|c|c|c|c|}
\hline \multirow[t]{3}{*}{ Condition } & \multirow[t]{3}{*}{ Variable } & \multicolumn{2}{|c|}{ Expectancies } & \multicolumn{2}{|c|}{ Alcohol } & \multicolumn{4}{|c|}{ Salience asymmetries } \\
\hline & & positive & negative & use & problems & alcohol-sodc & alcohol-soda & gative-positi & negative-positive \\
\hline & & & & & & same trials & different trials & same trials & different trials \\
\hline \multirow{2}{*}{$\begin{array}{l}\text { All } \\
(\mathrm{N}=46)\end{array}$} & D600 measure & -.19 & -.03 & $-.39^{* *}$ & $-.30^{*}$ & .18 & -.08 & .11 & .13 \\
\hline & ms measure & $-.49^{* *}$ & .04 & $-.50^{* *}$ & $-.34^{*}$ & $.37^{*}$ & .07 & .23 & .22 \\
\hline \multirow{3}{*}{$\begin{array}{l}\text { Familiar } \\
\text { alcohol } \\
\text { condition } \\
(\mathrm{N}=24)\end{array}$} & & & & & & & & & \\
\hline & D600 measure & -.08 & -.30 & $-.61^{* *}$ & -.36 & .22 & -.04 & .04 & -.04 \\
\hline & ms measure & -.29 & -.22 & $-.61^{* *}$ & $-.43^{*}$ & .23 & -.11 & .20 & .10 \\
\hline \multicolumn{10}{|l|}{ Unfamiliar } \\
\hline \multirow{2}{*}{$\begin{array}{l}\text { alcohol } \\
\text { condition } \\
(\mathrm{N}=22)\end{array}$} & D600 measure & -.25 & .05 & -.13 & -.24 & -.13 & -.38 & .11 & .21 \\
\hline & ms measure & $-.66^{* *}$ & .06 & -.35 & -.24 & .16 & -.07 & .17 & .14 \\
\hline
\end{tabular}

Note. Partial correlations are presented for the whole sample as well as separately for each familiarity condition. ${ }^{* *}=$ Correlation is significant at the 0.01 level (2-tailed), ${ }^{*}=$ Correlation is significant at the 0.05 level (2-tailed).

\section{Alcohol Expectancies}

Mean scores were calculated for the positive expectancy items and the negative expectancy items. Participants' mean score for the positive expectancies was significantly higher than the neutral midpoint of the scale, $t(45)=7.01, p<.001, d=1.03$, while their mean score for the negative expectancies was significantly lower than the neutral midpoint, $t(45)=-14.76, p<.001, d=2.18$. Hence, participants expected positive effects from drinking alcohol on explicit measures. Partial correlations between expectancies and IAT effects, controlled for order of IAT combination tasks, are presented in Table 2. Overall, only the negative correlation between the ms measure of IAT effects and positive expectancies reached statistical significance, indicating that participants who endorsed more positive alcohol expectancies also showed a smaller IAT effect. When partial correlations were calculated separately for the two familiarity conditions, the unfamiliar alcohol IAT was found to be negatively correlated with positive expectancies, although this was significant only for the conventional IAT measure, and uncorrelated with negative expectancies. The familiar alcohol IAT correlated negatively with both positive and negative expectancies although this was not statistically significant for both IAT measures. 


\section{Alcohol Use and Problems}

Estimates of alcohol use and alcohol-related problems were calculated as the sum scores of items 1 to 3 and items 4 to 10 in the AUDIT, respectively. The alcohol-related problems estimate was then log-transformed to obtain normality. Partial correlations of alcohol use and alcohol-related problems with IAT effects, controlled for order of IAT combination tasks, are presented in Table 2. Overall, the IAT was correlated negatively with both alcohol use and problems, indicating that participants who showed a smaller IAT effect consumed more alcohol and experienced more alcohol-related problems. When correlations were analyzed separately for the two familiarity conditions, only the familiar alcohol IAT was significantly correlated with both alcohol use and problems. In contrast, positive and negative expectancies were only borderline significantly correlated with alcohol use, $r=.28, p=.059$, and $r=-.25, p=.094$, respectively, and were uncorrelated with alcohol-related problems $(p>.30)$.

The FG account predicts that FG asymmetries will predict IAT effects above selfreported alcohol use and alcohol-related problems, and that the relationship between IAT effects and self-report measures will be attenuated by FG asymmetries. These predictions were tested in hierarchical multiple regression analyses. IAT effects were entered as the dependent variable in different regression analyses, performed separately for the two familiarity conditions, to examine whether entering FG asymmetries as a predictor of IAT effects would influence the prediction by alcohol use and alcohol-related problems. The first run of the hierarchical regression analysis for the familiar alcohol condition showed that selfreported alcohol use significantly predicted IAT effects in this condition and that entering FG asymmetries into the equation did not eliminate or attenuate this prediction. In the second run of the regression analysis, all variables that were not predictive of IAT effects $(p>.30)$ were left out. Results for this second run of the regression analysis again showed that alcohol use significantly predicted IAT effects in the familiar alcohol condition and that FG asymmetries between the alcohol and soda categories on same trails did not predict IAT effects nor did they eliminate the prediction of IAT effects by alcohol use (Table 3). In the unfamiliar alcohol condition, neither the self-report measures nor FG asymmetries significantly predicted IAT effects ${ }^{2}$. Next, it was examined whether the IAT predicted alcohol use above gender, age and explicit expectancies in the familiar alcohol condition. Note that in this hierarchical regression analysis, the IAT was entered as an independent variable instead of a dependent variable. This is because the hierarchical regression analyses reported above tested whether IAT effects were determined more by FG asymmetries than by selfreport measures in a manner similar to the analyses reported by Rothermund and Wentura (2004), whereas the following regression analysis examined the incremental validity of the familiar alcohol-IAT above explicit measures in the same way as Wiers et al. (2002).

\footnotetext{
${ }^{2}$ This table is not shown here, but is available upon request.
} 
Table 3

Summary of the hierarchical regression analysis for the prediction of IAT effects by self-reported alcohol use and alcohol-related problems and FG asymmetries, in the familiar alcohol condition.

\begin{tabular}{|c|c|c|c|c|c|c|c|}
\hline Step & Variable & B & SE B & $\beta$ & SE $\beta$ & $t$ & $p$ \\
\hline \multicolumn{8}{|c|}{ First run } \\
\hline \multirow[t]{2}{*}{1} & Alcohol use & -51.34 & 16.69 & -.58 & .19 & -3.08 & .006 \\
\hline & Alcohol-related problems & -15.30 & 58.07 & -.05 & .19 & -.26 & .795 \\
\hline \multirow[t]{6}{*}{2} & Alcohol use & -56.48 & 17.47 & -.64 & .20 & -3.23 & .005 \\
\hline & Alcohol-related problems & 13.98 & 63.37 & .05 & .21 & .22 & .828 \\
\hline & FG alcohol-soda same & 420.36 & 317.17 & .25 & .19 & 1.33 & .203 \\
\hline & FG alcohol-soda different & 186.43 & 499.05 & .07 & .19 & .37 & .713 \\
\hline & FG negative-positive same & 398.96 & 461.04 & .17 & .20 & .87 & .399 \\
\hline & FG negative-positive different & -114.42 & 396.84 & -.06 & .19 & -.29 & .777 \\
\hline \multicolumn{8}{|c|}{ Second run } \\
\hline 1 & Alcohol use & -53.12 & 14.93 & -.60 & .17 & -3.56 & .002 \\
\hline 2 & FG alcohol-soda same & 448.47 & 270.46 & .27 & .16 & 1.66 & .112 \\
\hline
\end{tabular}

Note. All variables that were not predictive $(p>.30)$ of IAT effects in the first run of the regression analysis were left out in the second run of the analysis. Results are shown for the conventional ms IAT effect measure. Results for the D600 IAT effect measure were generally the same and are available upon request. For the first run of the regression analysis: $F(2,21)=6.10, p=.008, R^{2}=.37$ for step $1 ; F_{\text {change }}(4,17)=.77, p=.562, R_{\text {change }}^{2}=.10$ for step 2 . For the second run of the analysis: $F(1,22)=12.67, p=.002, R^{2}=.37$ for step $1 ; F_{\text {change }}(1,21)=2.75, p=$ $.112, R_{\text {change }}^{2}=.07$ for step 2 .

In step 1 of the hierarchical regression analysis, gender significantly predicted alcohol use $(\beta=-.59, p=.008)$ while age did not $(\beta=-.32, p=.131), F(2,21)=4.35, p=.026$, $R^{2}=.29$. Positive and negative expectancies were entered in step 2 which did not increase the variance explained, $F_{\text {change }}(2,19)=.56, p=.580, R_{\text {change }}^{2}=.04$. Finally, the IAT was entered in step 3 and significantly predicted alcohol use the above background variables, both when the conventional ms IAT effect measure was entered as a predictor, $F_{\text {change }}(1$, $18)=8.12, p=.011, R_{\text {change }}^{2}=.21$, and when the D600 measure was used, $F_{\text {change }}(1$, $18)=.7 .89, p=.012, R_{\text {change }}^{2}=.20^{2}$.

\section{DISCUSSION}

Previous alcohol-IAT studies have repeatedly shown the counterintuitive finding that drinkers are faster to combine both alcohol and negatively valenced words than alcohol and positively valenced words in the IAT, while they, in contrast, indicate to be positive about alcohol on explicit measures (e.g., Wiers et al., 2002, 2005; Houben \& Wiers, 
2006c; this study). Here, it was tested whether this puzzling finding might reflect FG asymmetries, rather than meaningful associations between alcohol and negative valence. The FG account of the negative alcohol-IAT effect was tested in two ways. First, it was examined whether a familiarity manipulation designed to reverse FG asymmetries between IAT target categories would also reverse the negative alcohol-IAT effect as predicted by the FG account. Second, it was tested whether the IAT effect would be correlated with independently assessed FG asymmetries and whether these FG asymmetries would diminish the relationship between the IAT and alcohol use and alcohol-related problems.

Based on the present pattern of results it can be concluded that some aspects of the data corroborate the FG account of the alcohol-IAT while others do not. First, in line with the FG account, the familiarity manipulation influenced IAT effects in the expected direction: The negative alcohol-IAT effect was smaller in the familiar alcohol-IAT where both the (unfamiliar) soda target category and the negative attribute category were figures, than in the unfamiliar alcohol-IAT were the (unfamiliar) alcohol target category and the negative attribute category were figures. Also, the salience asymmetry between the alcohol and soda categories was correlated with the conventional ms measure of IAT effects, indicating that the ms IAT effect increased as alcohol became more salient than soda. Thus, conform the FG account, this correlation implies that it was easier to combine alcohol and negative words (which was always the figure category compared to the positive attribute category) in the IAT when alcohol was a figure category compared to soda. However, importantly and in contrast to the prediction by the FG account, the familiarity manipulation was insufficient to reverse the alcohol-negative IAT effect (as was demonstrated for other IATs by Rothermund \& Wentura, 2004). Furthermore, in support of the validity of the IAT, IAT effects in the familiar alcohol condition were predicted by selfreported alcohol use and FG asymmetries did not render this prediction nonsignificant. Also, the familiar alcohol-IAT predicted alcohol use above background variables and explicit expectancy measures. In contrast, the unfamiliar alcohol IAT was not predictive of alcohol use which is not surprising since the unfamiliar alcohol (and soda) targets presented in this IAT were probably drinks that were never consumed by the participants.

It is important to note that, whereas alcohol-IAT effects in the present study were influenced by the familiarity of the target concepts, other studies have not found noticeable effects of familiarity manipulations on results with ingroup-outgroup IATs (e.g., Dasgupta, McGhee, \& Greenwald, 2000; Ottaway, Hayden, \& Oakes, 2001; Rudman, Greenwald, Melliot, \& Schwartz, 1999). However, the aim of the present study was to examine the effect of FG asymmetries caused by differential familiarity of the target categories alcohol and soda on IAT effects. In contrast, Ottaway et al. (2001) contrasted either two familiar target categories or two unfamiliar target categories and, thus, did not create a FG asymmetry that was based on familiarity. Furthermore, studies by both Rudman et al. (1999) and Dasgupta et al. (2000) were mainly focused on examining whether IAT effects could be due to greater familiarity of ingroup stimuli compared to outgroup stimuli and 
demonstrated no influence of differential familiarity on IAT scores. However, in both studies, familiarity measures for both target categories were combined into a relative familiarity measure in such a way that higher scores indicated greater familiarity with ingroup stimuli rather than with outgroup stimuli. Hence, it is possible that outgroup stimuli, although less familiar than ingroup stimuli, were not sufficiently unfamiliar to create a FG asymmetry.

The difference in results with the D600 measure and the conventional ms measure is directly related to the ongoing discussion about the suitability of both measures as measures of IAT effects. In agreement with Greenwald et al. (2005), the present results suggest that the ms measure is more sensitive to FG effects than the D600 measure. However, similarly to what is argued by Rothermund et al. (2005), the present results also imply that the D-measure is not immune to FG effects.

A limitation to the present results is that valence was not controlled, which can also be a contributor to FG asymmetries in the IAT (Rothermund \& Wentura, 2004). Valence ratings of the alcohol and soda stimuli obtained from a subset of participants showed that there was no significant difference in valence between the familiar alcohol and unfamiliar soda categories, implying that salience asymmetries between this contrasted pair were based on the familiarity manipulation and not on valence. However, familiar soda stimuli were evaluated more positively than unfamiliar alcohol stimuli, implying that the unfamiliar alcohol category was more salient than the familiar soda category because it was both more unfamiliar and more negative than the familiar soda category.

The present results are consistent with previous alcohol-IAT studies in which familiarity of the target categories was properly matched (well known alcoholic drinks and sodas): the negative-alcohol IAT effect is typically only weakly correlated with direct selfreport measures of attitudes and expectancies (e.g., Wiers et al., 2002, 2005; Houben \& Wiers, 2006c) and has been found to be sensitive to differences in alcohol consumption (e.g., Wiers et al., 2002). Furthermore, with respect to the alcohol targets, the familiar alcohol IAT was highly similar to previous alcohol-IATs. However, the difference with previous alcohol-IATs concerned the now unfamiliar soda category. This manipulation resulted in a smaller IAT effect than reported in previous alcohol-IAT studies. In contrast, the unfamiliar alcohol IAT was similar to previous alcohol-IATs with respect to the (familiar) soda targets. Here, the IAT effect size was comparable to previous alcohol-IAT results. There are a number of reasons for this, including the possibility that a meaningful FG asymmetry could exist between the alcohol and soda categories in heavy drinkers because of an alcohol-related attentional bias that increases the salience of alcohol-related stimuli (see Field, Mogg, \& Bradley, 2006). Therefore, when using the alcohol-IAT, it is important to supplement the IAT with visual search tasks in order to assess possible FG asymmetries and to control for their influence on IAT effects. Nevertheless, the present results also show that FG asymmetries between the alcohol and soda target categories cannot completely 
account for the negative-alcohol IAT effect because no reversal of the IAT effect was found as would be predicted by the FG account.

Instead, the possibility was raised that the negative alcohol-IAT effect could be representative of problems related to alcohol use (e.g., Wiers et al., 2006). However, the direction of the correlation between the IAT and alcohol-related problems was not in agreement with this account. Also, the negative alcohol-IAT effect could reflect early, negative experiences with alcohol whereas explicit measures may be more related to recent experiences (Rudman, 2004). While this possibility was not tested in the present study, another study found no support for this hypothesis (Houben \& Wiers, 2006c). Another possibility could be that the IAT effect does not reflect an association between alcohol and negative expected outcomes, but between alcohol and a negative antecedent mood state. This temporal sequence of associations is not addressed by the IAT, but can be examined with other techniques such as semantic and affective priming procedures (cf. Zack, Toneatto, \& Macleod, 1999).

Future research should further examine the validity of the FG interpretation of IAT effects in different areas of experimental research, including psychopathology where FG asymmetries might be meaningful contributors to IAT effects. Also, researchers should remain attentive to the possible influence of salience asymmetries on IAT effects and therefore, in agreement with Rothermund and Wentura (2004), it is recommended to supplement the IAT with visual search tasks to examine whether salience asymmetries are present within the IAT dimensions and to control for salience effects. 


\section{APPENDIX}

\section{IAT Target Stimuli}

Alcohol (familiar): beer, wine, baileys, bacardi, martini

Alcohol (unfamiliar): vieux, absinth, batida de coco, tia maria, fiero

Soda (familiar): ice tea, apple juice, fanta, spa (mineral water), coke

Soda (unfamiliar): roosvicee (syrup), karvan cevitam (syrup), water joe, breaker (yoghurt drink), carrot juice

\section{IAT Attribute Stimuli}

Positive: social, good, pleasant, nice, enjoyable

Negative: antisocial, bad, unpleasant, stupid, obnoxious 


$$
\text { D) }
$$




\begin{abstract}
Research using the Implicit Association Test (IAT) repeatedly demonstrated negative implicit alcohol associations regardless of level of alcohol use. However, since the IAT is susceptible to recoding influences, this finding could reflect recoding processes rather than implicit alcohol associations. Here, implicit alcohol associations were assessed with the newly developed IAT-RF (IAT-Recoding Free) that prevents recoding processes by switching response assignments randomly between trials rather than between blocks. Moreover, this study was the first to examine the IAT-RF's predictive validity. IAT-RF effects were examined separately for repetition and switch trials (trials that respectively repeated or switched the response assignment of the preceding trial). While IAT-RF repetition trials yielded moderate support for negative associations with alcohol that correlated negatively with alcohol use, effects in IAT-RF switch trials were not significant but correlated positively with alcoholrelated problems. These results suggest that previous findings with the IAT partly reflect recoding processes. Importantly, recoding-free implicit alcohol associations predicted alcohol-related behavior above explicit alcohol-related expectancies. These findings also suggest that the two different IAT-RF trial types measure different underlying constructs.
\end{abstract}

\title{
INTRODUCTION
}

Contemporary models of addictive behaviors stress that cognitive processes underlying addictive behaviors can develop automaticity with increased substance use (e.g., Bechara, Noel, \& Crone, 2006; Deutsch \& Strack, 2006; Evans \& Coventry, 2006). Importantly, the increased understanding of addictive behaviors in terms of automatic processes has stimulated the use of indirect measures ${ }^{3}$ which, compared to traditionally used self-report measures, seem better suited for assessing cognitions that can influence behavior automatically (e.g. De Houwer, 2006; De Houwer \& Moors, in press).

One of the indirect measures that received most attention over the last years is the Implicit Association Test (IAT; Greenwald, McGhee, \& Schwartz, 1998). In the IAT, participants have to classify stimuli into two target categories and two attribute categories using two response keys. The idea is that this classification task should be easier when the

\footnotetext{
${ }^{3}$ Indirect measures are commonly referred to as implicit measures. However, the denotation of the term "implicit" remains confusing and is typically used interchangeably to denote both measurement procedures as well as outcomes of measurement procedures (De Houwer, 2006). For reasons of clarity, we will use the terms "indirect" and "direct" when referring to measurement procedures and the terms "implicit" and "explicit" to denote cognitions assessed with indirect and direct measures, respectively.
} 
response assignment of the target and attribute categories is compatible, or corresponds to respondents' implicit memory associations (e.g., "flowers" and "pleasant" vs. "insects" and "unpleasant"), than when the response assignment is incompatible, or does not match respondents' implicit memory associations (e.g., "flowers" and "unpleasant" vs. "insects" and "pleasant"). Hence, the performance difference between the two response assignments should reflect the strength of the associations between the target categories and the attribute categories. Over the past years, studies that used the IAT to study implicit associations that might be involved in alcohol use and abuse have repeatedly demonstrated faster performance when the concepts "alcohol" and "negative" (vs. "soda" and "positive") are assigned to the same response than when the concepts "alcohol" and "positive" (vs. "soda" and "negative") share a response (e.g., De Houwer, Crombez, Koster, \& De Beul, 2004; Wiers, van de Luitgaarden, van den Wildenberg, \& Smulders, 2005; Wiers, van Woerden, Smulders, \& de Jong, 2002). Importantly, these IAT effects were at best only moderately related to drinking behavior, and in clear contrast with respondents' explicit positive alcohol-related cognitions.

Although a myriad of research has been devoted to the IAT, showing that it can offer both a reliable and valid measure of automatically activated associations (e.g., Cunningham, Preacher, \& Banaii, 2001; Hofmann, Gawronski, Gschwendner, Le, \& Schmitt, 2005; Nosek, Greenwald, \& Banaii, 2006), uncertainty remains as to what the IAT exactly measures. Specifically, it was suggested that compatibility effects in the IAT are not necessarily based on implicit memory associations but can also be influenced by recoding processes (Greenwald \& Nosek, 2001; Nosek \& Hansen, 2007; Rothermund, Wentura, \& De Houwer, 2005; for evidence see, e.g., De Houwer, Geldof, \& De Bruycker, 2005; Mierke \& Klaver, 2001, 2003; Rothermund \& Wentura, 2001, 2004). Recoding means that target and/or attribute stimuli are categorized based on features that do not match the "nominal definitions" of the target (e.g., "alcohol" vs. "soda") and/or attribute categories (e.g., "positive" vs. "negative"; cf. Greenwald, Nosek, Banaii, \& Klaver, 2005).

Recoding influences on IAT effects are problematic mainly because they tend to obscure the nature of the processes underlying compatibility effects in the IAT. It is often not clear whether recoding of the categorization task has actually occurred, and if so, what kind of recoding has taken place. It is therefore difficult to interpret the meaning of compatibility effects in the IAT because it is unclear with regard to which feature the target and attribute categories are compatible ${ }^{4}$. For example, it certainly makes a difference for the interpretation of an IAT effect whether the categories "alcohol" and "negative" (vs. "soda" and "positive") are compatible, indicating that "negative" ("positive") is an intrinsic

\footnotetext{
${ }^{4}$ Recoding does not require conscious strategies but can also reflect automatic learning of covariations between categories, features, and responses (cf. Lewicki, Hill, \& Czyzewska, 1992). Due to the subtlety of the recoding process, it is often impossible to identify the features (or combinations of features) that are responsible for a given compatibility effect in the IAT.
} 
feature of the mental representation of the category "alcohol" ("soda") that is automatically activated whenever stimuli are categorized as "alcohol" ("soda"), or whether familiarity, valence, salience, or any other feature was used to discriminate between both pairs of categories (Greenwald \& Nosek, 2001; Rothermund et al., 2005; Wentura \& Rothermund, in press).

Houben and Wiers (2006a) recently demonstrated that recoding in terms of salience contributes to effects in the alcohol-IAT. However, it was also evident that this kind of recoding could not completely account for the IAT effect as reversing salience compatibilities only influenced the size but not the direction of the IAT effect. Nevertheless, these findings do not exclude the possibility that other types of recoding processes are operating in the alcohol-IAT. To overcome this problem of the IAT, Rothermund, TeigeMocigemba, and Wentura (2006) recently introduced a modified version of the IAT, which they termed IAT-RF (short for "IAT-Recoding Free"). Basically, the IAT-RF aims to eliminate effects of recoding by abandoning the block structure of the standard IAT. In the standard IAT, compatible and incompatible trials are presented in separate blocks of the task. This structure creates an extended consistent mapping of categories onto responses in the compatible block, which may invite recoding processes. In the IAT-RF, in contrast, compatible and incompatible response assignments of categories vary randomly between trials within a single block. This random switching of response assignments undermines any type of recoding, because efficient recoding requires a consistent mapping of categories onto responses (Roßnagel, 2001; Shiffrin \& Schneider, 1977; Strayer \& Kramer, 1994). Rothermund et al. (2006) found that the IAT-RF efficiently eliminated artificial compatibility effects in a geometry IAT (Mierke \& Klaver, 2003) that depend on a recoding of the target color categories (e.g., "red" vs. "blue") in terms of confounded attributes ("large" vs. "small"). Also, the IAT-RF produced reliable compatibility effects between the target categories "flowers" vs. "insects" and the attribute categories "good" vs. "bad", which establishes the sensitivity of the measure for assessing existing compatibilities. Together, these findings attest to the validity of the IAT-RF as a pure measure of compatibilities between nominal categories. Although they did not yet investigate the predictive validity of the IAT-RF empirically, Rothermund et al. (2006) proposed that the IAT-RF might be wellsuited to assess interindividual differences in the strength of compatibility effects that are not contaminated by recoding processes.

Since it remains unclear to what extent recoding processes influence results with the alcohol-IAT, the IAT-RF might prove to be a valuable alternative to the IAT for studying the cognitive processes underlying alcohol use and might provide important insights regarding previous findings from studies that used the standard IAT. Importantly, if recoding processes influence performance in the alcohol-IAT, this could reduce the validity of the IAT as a measure of implicit alcohol-related associations as well as its predictive validity with respect to drinking behavior. Therefore, in this study, we examined whether the IAT-RF would also show a compatibility effect indicating faster performance when alcohol was 
paired with negative stimuli (vs. soda and positive stimuli) than when alcohol was paired with positive stimuli (vs. soda and negative stimuli) as was previously found with the standard IAT. If this finding is not replicated with the IAT-RF, this would suggest that previously reported IAT results to some extent reflect recoding processes. In addition, we examined whether IAT-RF trials that repeated the response assignment of the preceding trial and IAT-RT trials that switched the response assignment compared to the preceding trial show the same pattern of results, because recoding could be more efficiently eliminated in response assignment switch trials than in response assignment repetition trials, where recoding processes could still be operating on a "micro-level". Importantly, since the elimination of recoding processes in the IAT should increase its predictive validity, we expected a strong relationship between recoding-free implicit alcohol associations assessed with the IAT-RF and drinking behavior. More specifically, it was expected that stronger positive implicit alcohol associations (or weaker negative implicit alcohol associations) would predict an increase in alcohol consumption. Finally, it should be noted that this study was the first to empirically examine the predictive validity of the IAT-RF.

\section{METHOD}

\section{Participants}

Fifty-four male students (mean age $=22$ years, $S D=4.73$ ) of Maastricht University participated in exchange for a gift certificate. Average alcohol consumption per week was 17.93 (SD = 11.28) Dutch standard drinks. On the 18-items version of the Rutgers Alcohol Problem Index (RAPI; White \& Labouvie, 2000), the average item score was 76 $(S D=.36)$, while the average item score within clinical samples is about .80 (White \& Labouvie, 1989). On the Alcohol Use Disorder Identification Test (AUDIT; Saunders, Aasland, Babor, De la Fuente, \& Grant, 1993), participants' mean score was 13.83 (SD $=3.83$ ), while the proposed cut-off score for the screening of alcohol-related problems is 11 (Saunders et al., 1993).

\section{Materials and Measures}

Alcohol Use \& Alcohol-Related Problems. Alcohol use was measured with a self-report questionnaire (Wiers, Hoogeveen, Sergeant \& Gunning, 1997) based on the timeline follow-back method (Sobell \& Sobell, 1990). Participants were asked to indicate how many drinks of different types of alcoholic beverages they consumed during each day of the past week, and how many drinks they typically consumed on each day of the week. Alcohol-related problems were assessed with the RAPI and the AUDIT. The RAPI described 18 alcohol-related problems or situations for which participants indicated how often they experienced these situations on a 5 -point Likert scale $(0=$ never, 4 = very often) 
(Cronbach's $\alpha=.72$ ). The AUDIT consisted of 10 questions in a multiple choice format ( $\alpha$ $=.70$ ). The first three questions were related to alcohol use, the other seven to alcoholrelated problems.

The IAT-RF. The two target categories in the IAT-RF consisted of 5 alcohol ("alcohol") and 5 soda ("soda") stimuli ${ }^{5}$. The two attribute categories consisted of 5 positive words that were also rated high on arousal ("active") and 5 negative words that were also rated low on arousal ("miserable") 6 . All stimuli are presented in the Appendix. The IAT-RF was programmed in ERTS 3.18 (Beringer, 1996) and consisted of three blocks. In the first block, participants practiced the attribute classification using a left and a right response key (e.g., "active" vs. "miserable"). In second block, participants received 20 trials of target discrimination practice during which response assignments (e.g., "alcohol" vs. "soda" and "soda" vs. "alcohol") were randomly switched between trials with the restriction that each target stimulus was presented once in each response assignment. Finally, participants received 160 combination trials during which they had to classify both target and attribute stimuli with switching response assignments for the target categories. As during the target discrimination practice, response assignments were switched randomly between trials with the restriction that half the trials were presented in one response assignment (e.g., "active" and "alcohol" vs. "soda" and "miserable") and the other half of the trials in the other response assignment ("active" and "soda" vs. "miserable" and "alcohol"). Instructions were presented before each block. Target and attribute stimuli were presented on a strictly random basis in the middle of the computer screen in black against a grey background. Stimuli remained on screen until a response was given. Category labels were presented on the left and the right of the presented stimulus in accordance with the response assignment, so that participants would not need to make large eye movements to grasp the labels. Labels were presented at the start of each trial, $1500 \mathrm{~ms}$ before the upcoming stimulus, and disappeared after a response was given. The intertrialinterval was $250 \mathrm{~ms}$. Feedback was presented in red beneath the stimulus after an incorrect response ('wrong'), and when responses were too fast (<300 ms; 'too fast') or too slow (> $3000 \mathrm{~ms}$; 'too slow').

\footnotetext{
${ }^{5}$ The alcohol and soda target categories were matched on familiarity $(M=5.14, S D=1.24$, and $M=4.87, S D=$ 1.44 , respectively; $1=$ completely unfamiliar, $7=$ very familiar), valence $(M=4.50, S D=.82$, and $M=4.54, S D$ $=.83$, respectively; $1=$ extremely negative, $7=$ extremely positive), arousal $(M=4.38, S D=.87$, and $M=4.64$, $S D=1.00$, respectively; $1=$ extremely passive, $7=$ extremely active), and number of syllables.

${ }^{6}$ We selected positive $(M=6.14, S D=.55)$ and negative $(M=1.54, S D=.50)$ attributes that respectively scored high $(M=6.30, S D=.45)$ and low $(M=1.91, S D=.54)$ on arousal, so that these attribute categories would resemble alcohol-related positive reinforcement and negative alcohol-related expectancies, respectively, which have been demonstrated to be important positive and negative predictors of drinking behavior, respectively (cf. Wiers et al., 2006).) Both attribute categories were matched on familiarity as closely as possible $(M=5.79, S D=.77$, and $M=3.72, S D=1.65$, respectively) and number of syllables.
} 
Alcohol-Related Expectancies. Explicit expectancies with respect to drinking alcohol were measured with an expectancy questionnaire consisting of 6 positive expectancy items $(\alpha=.89)$, and 6 negative expectancy items $(\alpha=.76)$. Each item asked participants to indicate on a $100 \mathrm{~mm}$ Visual Analogue Scale (VAS) how much they agreed $(0=$ completely disagree, $100=$ completely agree) with the statement "After drinking alcohol, I feel ...", which was completed with the same words as presented in the IAT-RF, including the labels.

\section{Procedure}

Participants performed the IAT-RF first. The response assignment of the attribute categories in the IAT-RF was counterbalanced. Next, participants filled out the alcohol expectancy questionnaire, the alcohol use questionnaire, the AUDIT and the RAPI. Finally, participants rated all stimuli on familiarity, valence and arousal.

\section{RESULTS}

\section{Compatibility Effects in the IAT-RF}

Trials with incorrect responses (7\%) were discarded from the analyses. We will focus only on results for the target trials, first, because we are only interested in the target classification and not in the attribute classification, and second, because the labels switched only with respect to the target categories. Trials with response latencies below $300 \mathrm{~ms}$ or above $3000 \mathrm{~ms}$ were recoded to these boundaries, respectively (cf. Greenwald et al., 1998). Response latencies were log-transformed and aggregated for each participant and for each condition of the within-subjects design. Results were analyzed using a 2 (response assignment: compatible or incompatible) $\times 2$ (response assignment shift: switch or repetition) repeated measures analysis of variance.

Results showed a main effect for response assignment shift, $F(1,53)=6.05, p=$ .017 , but no significant compatibility effect, $F(1,53)=1.57, p=.216$. In addition, the interaction between compatibility of the response assignment and response assignment shift was significant, $F(1,53)=5.04, p=.029$ (see Figure 1). When the compatibility effect was examined separately for response assignment repetition and switch trials, results showed a borderline significant compatibility effect for response assignment repetition trials, $F(1,53)=3.65, p=.061$, indicating faster performance for compatible response assignment ("alcohol" and "miserable" vs. "soda" and "active") compared to the incompatible response assignment ("soda" and "miserable" vs. "alcohol" and "active"). However, there was no compatibility effect for response assignment switch trials, $F(1,53)$ $=.44, p=.509$. 


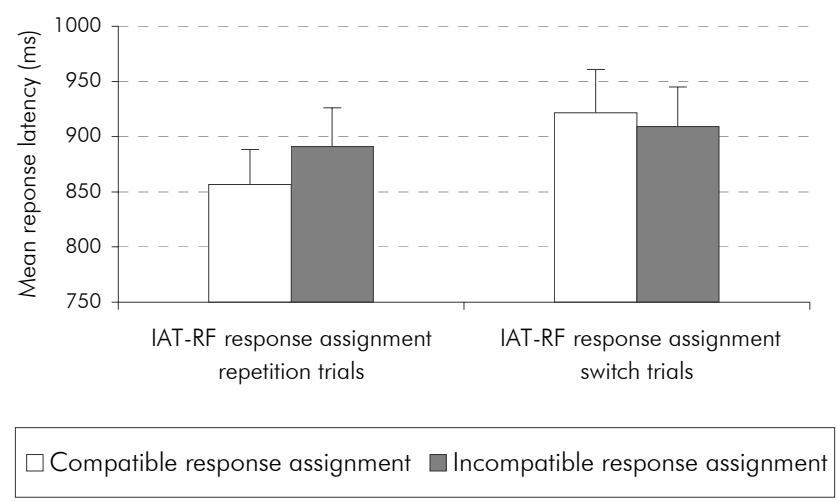

Figure 1. Mean response latencies for the compatible and incompatible response assignments, separately for IAT-RF response assignment repetition trials and IAT-RF response assignment switch trials.

Compatibility effect scores were calculated by subtracting mean log-transformed response latencies during the compatible response assignment from mean log-transformed response latencies during the incompatible response assignment, separately for response assignment repetition and switch trials. Hence, positive IAT-RF scores indicate faster performance when "alcohol" was paired with "miserable" (vs. "soda" and "active") than when "alcohol" was paired with "active" (vs. "soda" and "miserable"). As shown in Table 1, effect scores for response assignment repetition trials and effect scores for response assignment switch trials were uncorrelated.

Table 1

Correlations of IAT-RF scores ${ }^{a}$ with explicit alcohol-related expectancies, and self-reported alcohol use and alcoholrelated problems.

\begin{tabular}{|c|c|c|}
\hline \multirow[b]{2}{*}{ Variable } & \multicolumn{2}{|c|}{ IAT-RF } \\
\hline & $\begin{array}{l}\text { Response assignment } \\
\text { repetition trials }\end{array}$ & $\begin{array}{c}\text { Response assignment } \\
\text { switch trials }\end{array}$ \\
\hline IAT-RF switch trials & .25 & - \\
\hline Positive alcohol expectancies & .00 & .06 \\
\hline Negative alcohol expectancies & -.02 & .13 \\
\hline Alcohol use & $-.28^{*}$ & .11 \\
\hline Alcohol-related problems & -.20 & $.30^{*}$ \\
\hline
\end{tabular}




\section{Relationship of the IAT-RF to Explicit Expectancies and Alcohol- Related Behavior}

Mean expectancy scores were calculated separately for the negative $(M=23.41, S D=$ 9.87) and positive $(M=68.78, S D=11.75)$ items of the alcohol expectancy questionnaire. Similarly, we also calculated a mean score for alcohol-related attitudes $(M$ $=61.36, S D=8.85)$. As can be seen in Table 1, none of the effects calculated for the IAT-RF repetition and switch trials correlated significantly with explicit alcohol-related expectancies.

An estimate of alcohol use was calculated as the weighed mean of alcohol consumption during the past week and average weekly alcohol consumption. Similarly, an estimate of alcohol-related problems was calculated as the mean of the z-transformed RAPI and AUDIT sum scores. These estimates of alcohol use and alcohol-related problems were significantly correlated, $r=.47, p<.001$. Correlations of alcohol use and alcoholrelated problems with IAT-RF effect scores are presented in Table 1. IAT-RF repetition trials were negatively related to alcohol use and unrelated to alcohol-related problems. Hence, increased alcohol consumption is related to weaker compatibility effects in IAT-RF repetition trials. Conversely, effects for IAT-RF switch trials were unrelated to alcohol use, while they were positively correlated with alcohol-related problems, indicating that participants who experienced more alcohol-related problems, more easily paired alcohol with negative than with positive during IAT-RF switch trials.

Table 2

Summary of hierarchical regression analysis for the prediction of self-reported alcohol use by explicit alcohol-related expectancies and IAT-RF effect scores ${ }^{\mathrm{a}}$.

\begin{tabular}{llcccccc}
\hline Step & Variable & $B$ & SE B & $\beta$ & SE $\beta$ & $t$ & $p$ \\
\hline 1 & Positive alcohol expectancies & .23 & .13 & .26 & .15 & 1.74 & .088 \\
& Negative alcohol expectancies & -.05 & .16 & -.04 & .15 & -.30 & .769 \\
2 & Positive alcohol expectancies & .21 & .13 & .23 & .14 & 1.61 & .115 \\
& Negative alcohol expectancies & -.09 & .16 & -.09 & .14 & -.59 & .557 \\
& IAT-RF repetition trials & -22.94 & 9.39 & -.33 & .13 & -2.44 & .018 \\
& IAT-RF switch trials & 24.96 & 17.94 & .19 & .14 & 1.39 & .170
\end{tabular}

Note. $F(2,51)=2.14, p=.128, R^{2}=.08$, for step $1 ; F_{\text {change }}(2,49)=3.30, p=.045, R_{\text {change }}^{2}=.11$, for step 2 . Final model: $R^{2}=.19, R_{\text {adiusted }}^{2}=.12, F(4,49)=2.82, p=.035 ;{ }^{\circ}$ IAT-RF compatibility effects were scored so that positive values indicate faster responses if "alcohol" and "miserable" (and "soda" and "active") were assigned the same response.

Next, we tested the incremental validity of the IAT-RF. The hierarchical regression analysis predicting alcohol use is shown in Table 2. Explicit alcohol-related expectancies were entered in step 1 and only positive alcohol-related expectancies borderline significantly 
predicted alcohol use. In step 2, compatibility effects for IAT-RF repetition trials significantly predicted alcohol use whereas compatibility effects for IAT-RF switch trials did not. In the final model, only the IAT-RF effect for response assignment repetition trials significantly predicted alcohol use. The same procedure was followed for the prediction of alcoholrelated problems (see Table 3). In step 1, negative alcohol-related expectancies significantly predicted alcohol-related problems while positive alcohol-related expectancies were borderline significant predictors of alcohol-related problems. In step 2, IAT-RF compatibility effects in both repetition trials and switch trials significantly predicted alcoholrelated problems above alcohol-related expectancies. However, since the results from the hierarchical regression analyses demonstrate that effects in IAT-RF repetition trials predict both alcohol use and alcohol-related problems, and since alcohol use and alcohol-related problems were strongly correlated, it was suspected that the relationship between effects in repetition trials and alcohol-related problems might be mediated by alcohol use.

Table 3

Summary of hierarchical regression analysis for the prediction of self-reported alcohol-related problems by explicit alcohol-related expectancies, IAT-RF effect scores ${ }^{\circ}$, and alcohol use.

\begin{tabular}{|c|c|c|c|c|c|c|c|}
\hline Step & Variable & B & SE B & $\beta$ & SE $\beta$ & $t$ & $p$ \\
\hline \multirow[t]{2}{*}{1} & Positive alcohol expectancies & .02 & .01 & .24 & .14 & 1.76 & .085 \\
\hline & Negative alcohol expectancies & .04 & .01 & .48 & .14 & 3.43 & .001 \\
\hline \multirow[t]{4}{*}{2} & Positive alcohol expectancies & .02 & .01 & .20 & .13 & 1.52 & .135 \\
\hline & Negative alcohol expectancies & .04 & .01 & .41 & .13 & 3.11 & .003 \\
\hline & IAT-RF repetition trials & -1.63 & .74 & -.27 & .12 & -2.19 & .033 \\
\hline & IAT-RF switch trials & 3.34 & 1.42 & .30 & .13 & 2.36 & .023 \\
\hline \multirow[t]{5}{*}{3} & Positive alcohol expectancies & .01 & .01 & .10 & .12 & .80 & .426 \\
\hline & Negative alcohol expectancies & .04 & .01 & .45 & .12 & 3.87 & .000 \\
\hline & IAT-RF repetition trials & -.73 & .69 & -.12 & .11 & -1.05 & .298 \\
\hline & IAT-RF switch trials & 2.36 & 1.27 & .21 & .11 & 1.86 & .069 \\
\hline & Alcohol use & .03 & .01 & .46 & .12 & 3.95 & .000 \\
\hline
\end{tabular}

Mediation was tested following the steps described by Baron and Kenny (1986). Importantly, when alcohol use was also entered as a predictor in the hierarchical regression analysis for the prediction of alcohol-related problems (in step 3; see Table 3), results confirmed that the relationship between effects in IAT-RF repetition trials and 
alcohol-related problems was completely mediated by alcohol use as effects for IAT-RF repetition trials were no longer a significant predictor of alcohol-related problems when controlling for alcohol use. In the final model, alcohol-related problems were significantly predicted by both negative expectancies and alcohol use and borderline significantly predicted by effects for IAT-RF switch trials.

\section{DISCUSSION}

The aim of the present study was to examine whether previously reported results with the alcohol-IAT, demonstrating that alcohol is more easily paired with negative stimuli than with positive stimuli (e.g., De Houwer et al., 2004; Wiers et al., 2002, 2005), were due to recoding effects rather than implicit associations with alcohol. We therefore examined whether these results could be replicated with the IAT-RF, which is designed to eliminate recoding processes by varying compatible and incompatible response assignments randomly on a trial-by-trial basis instead of in separate blocks (Rothermund et al., 2006). The present study was also the first to empirically examine the predictive validity of the IAT-RF.

First, the present results suggest that previous findings with the alcohol-IAT at least partly reflected recoding processes. Whereas previous studies found robust and strong IAT effects due to faster performance when alcohol was paired with negative attributes compared to positive attributes (e.g., De Houwer et al., 2004; Wiers et al., 2002, 2005), the present results, at best, only demonstrate moderate effects in the same direction. Specifically, IAT-RF effects calculated from response assignment repetition trials were only borderline significant while IAT-RF effects calculated from response assignment switch trials were not significant. Together, results suggest that the alcohol-IAT used in previous studies was to some extent influenced by recoding processes which increased the size of IAT compatibility effects. Elimination of these recoding processes caused compatibility effects in this study to decrease (IAT-RF repetition trials) and even disappear (IAT-RF switch trials), which could indicate that participants on average did not have any associations between alcohol and valence (neutral). Alternatively, these results could indicate that alcohol can simultaneously be associated with both positive and negative valence. Consistent with this interpretation, recent studies in which IAT attribute categories were presented in a unipolar format (e.g., "pleasant" vs. "neutral"), have demonstrated that positive and negative associations with alcohol can co-exist (e.g., Jajodia \& Earleywine, 2003; Houben \& Wiers, 2006c, 2006d).

Second, IAT-RF effects in repetition trials were negatively related to alcohol use and predicted alcohol use better than explicit alcohol-related expectancies. The same relationship between alcohol-related associations and alcohol use was also demonstrated when IAT effects were controlled for recoding in terms of salience asymmetries (Houben \& Wiers, 2006a). These results suggest that stronger positive alcohol associations, or weaker negative alcohol associations, are associated with increased alcohol use. Importantly, recent research 
in which unipolar IATs were used to measure alcohol-related associations suggests that increased levels of alcohol use are associated with stronger positive associations with alcohol while negative associations with alcohol are unrelated to alcohol consumption (Houben \& Wiers, 2006c, 2006d). In contrast, compatibility effects in IAT-RF switch trials were unrelated to alcohol use but positively related to alcohol-related problems and also predicted alcoholrelated problems above explicit measures. These results are consistent with the idea that negative alcohol-related cognitions increase with the experience of alcohol-related problems but do not influence drinking until a certain threshold is reached, which was supported with respect to explicit alcohol-related cognitions (Jones \& McMahon, 1996), but not yet with respect to implicit alcohol-related cognitions although there is indirect supportive evidence (cf. Wiers, Houben, Smulders, Conrod, \& Jones, 2006).

Finally, it is important to note that IAT-RF repetition trials and IAT-RF switch trials showed different patterns of results in this study, especially with respect to their relationship with drinking behavior. To explain this difference, one could assume that recoding was efficiently eliminated in IAT-RF switch trials but still present to some extent in repetition trials possibly due to recoding taking place on a "micro-level" (i.e., whenever response assignments are repeated, the consistency of the mapping might allow for some recoding). If recoding processes were still operating in IAT-RF repetition trials, this could explain why IATRF repetition trials, but not IAT-RF switch trials, yielded compatibility effects, which were reduced but in the same direction as effects reported with the standard alcohol-IAT. For now it seems safe to conclude that the two different types of trials in the IAT-RF, namely response assignment repetition trials and response assignment switch trials, possibly measure different underlying cognitive constructs. Therefore, an important implication of these results is that it is useful to analyze IAT-RF repetition and switch trials separately. Finally, given the potential value of the unipolar IAT for assessing compatibilities for concepts for which ambivalence is likely to be high (e.g., addictive substances) it would be interesting for future research to examine how recoding influences the unipolar IAT and whether the unipolar IAT, like the IAT, can be adapted to be free from recoding effects.

In conclusion, the present findings indicate that recoding processes can influence IAT effects and thereby decrease the validity of the task as a measure of implicit associations that may be involved in alcohol abuse. Importantly, preventing or controlling for recoding processes in the IAT increases the validity of the IAT, making it a useful tool for studying cognitive processes underlying alcohol use and misuse. Here, it was demonstrated that stronger implicit alcohol associations with positive arousing effects are related to increased alcohol consumption. Hence, these results indicate that intervention strategies aimed at reducing hazardous drinking may be more successful when they simultaneously target both explicit alcohol-related cognitive processes and implicit alcohol-related cognitive processes. 


\section{APPENDIX}

\section{Target Stimuli}

Alcohol: beer, wine, whisky, drink, vodka

(Dutch words; Alcohol: bier, wijn, whisky, borrel, vodka)

Soda: Fanta, Coke, Sprite, sinas (lemonade), ice-tea

(Dutch words; Frisdrank: Fanta, Cola, Sprite, sinas, ice-tea)

\section{Attribute Stimuli}

Active: cheerful, energetic, lively, excited, chatty

(Dutch words; Actief: vrolijk, energiek, levendig, opgewonden, spraakzaam)

Miserable: cheerless, horrible, nauseous, unhappy, listless

(Dutch words; Beroerd: somber, ellendig, misselijk, ongelukkig, lusteloos) 


$$
\text { D) }
$$




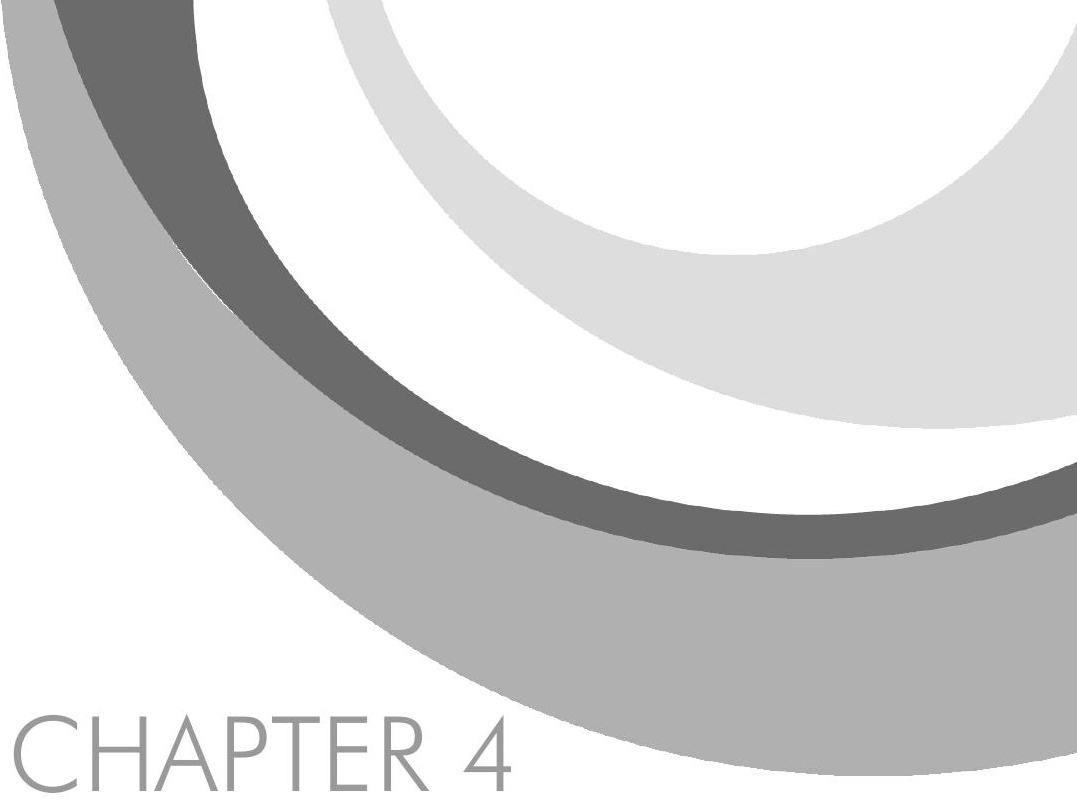

\section{Are Drinkers Implicitly Positive about Drinking Alcohol? Personalizing the Alcohol-IAT to Reduce Negative Extrapersonal Contamination}

Adapted version of: Houben, K., \& Wiers, R. W. (2006). Are Drinkers Implicitly Positive about Drinking Alcohol? Personalizing the Alcohol-IAT to Reduce Negative Extrapersonal Contamination. Accepted for publication pending revisions. 


\begin{abstract}
Aims: The advent of indirect measures, such as the Implicit Association test (IAT), has stimulated the interest in implicit cognitions that may automatically steer addictive behaviors such as alcohol abuse. Counter intuitively, recent IAT research demonstrated that alcohol is implicitly associated with negative valence, regardless of level of alcohol consumption. However, because the IAT is susceptible to extrapersonal contamination, this study examined whether previous findings reflect contamination of IAT effects by negative extrapersonal knowledge rather than personal associations that are relevant to drinking behavior. Methods: Implicit alcohol associations were measured with a personalized alcohol-IAT, designed to reduce extrapersonal contamination. It was examined whether alcohol associations measured with the personalized IAT would predict drinking behavior above the variance explained by self-reported alcohol-related expectancies and attitudes. Results: In contrast to previous findings with the IAT, the personalized IAT yielded positive associations. Moreover, positive alcohol associations predicted drinking behavior above self-reported alcohol expectancies and attitudes, demonstrating the incremental validity of the personalized IAT. Conclusions: The present findings support the hypothesis that previous findings with the alcohol-IAT at least partly reflect negative extrapersonal alcoholrelated knowledge and that behavior-relevant implicit alcohol associations are positive rather than negative.
\end{abstract}

\title{
INTRODUCTION
}

The past years, the advent of indirect measures has stimulated a growing interest in the role of implicit cognitions in the etiology and maintenance of addictive behaviors such as alcohol abuse. The reason is that, with increased substance abuse, cognitive processes underlying the addictive behavior develop automaticity and these automatic or implicit processes subsequently determine behavior more than explicit, more controlled cognitive processes (e.g., Deutsch \& Strack, 2006; Stacy, 1997). Importantly, these automatic processes that determine alcohol use are difficult, though not impossible, to assess with direct self-report measures. Moreover, direct measures can be influenced by biasing factors such as self-presentation (e.g., Holtgraves, 2004; Paulhus, 1984). In contrast, indirect measures, which infer cognitive processes indirectly from behavior other than selfreport, seem more resistant to self-presentation biases and may be uniquely suited to tap the automatic influence of alcohol-related cognitions on behavior (e.g. De Houwer, 2006; De Houwer \& Moors, in press). 
In a few years, the Implicit Association Test (IAT; Greenwald, McGhee, \& Schwarz, 1998) has become one of the most frequently applied indirect measures in many areas of research including addiction research. The IAT is a speeded classification task that involves participants classifying stimuli into two target categories and two attribute categories using two response keys. The idea is that this classification task should be easier when the response assignment of the target and attribute categories is compatible, or corresponds to respondents' implicit associations (e.g., flowers and positive vs. insects and negative), than when the response assignment is incompatible, or does not match respondents' implicit associations (e.g., flowers and negative vs. insects and positive). Hence, the performance difference between the two response assignments is assumed to reflect the strength of the associations of the target categories with the attribute categories. Wiers, van Woerden, Smulders, and de Jong (2002) were the first to apply the IAT to study implicit alcohol-related cognitions in light and heavy drinkers. Wiers et al. found that both light and heavy drinkers responded faster when alcohol shared a response with negative (vs. soda and positive) than when alcohol was paired with positive (vs. soda and negative), indicating that both light and heavy drinkers associated alcohol with negative valence in the IAT. Importantly, these IAT scores contrasted with participants' self-reported positivity about drinking alcohol. Since then, these results have been also replicated in heavy drinkers (Wiers, van de Luitgaarden, van den Wildenberg, \& Smulders, 2005) and in drinkers (De Houwer, Crombez, Koster, \& De Beul, 2004).

Though the IAT can offer both a reliable and valid measure of implicit associations (e.g., Cunningham, Preacher, \& Banaji, 2001; Hofmann, Gawronski, Gschwendner, Le, \& Schmitt, 2005; Nosek, Greenwald, \& Banaji, 2006; Poehlman, Uhlmann, Greenwald, \& Banaji, 2005), recent research suggests that the IAT is not only influenced by implicit associations but by any information that can facilitate IAT performance (e.g., De Houwer, Geldof, \& De Bruycker, 2005; Mierke \& Klaver, 2003; Rothermund, Teige-Mocigemba, \& Wentura, 2006; Rothermund \& Wentura, 2004; for the alcohol-IAT see also Houben, Rothermund, \& Wiers, 2006; Houben \& Wiers, 2006a). Related to this issue, it was recently demonstrated that the IAT is sensitive to extrapersonal associations that are available in memory but that do not form the basis of one's attitudes and that are irrelevant to behavior. Extrapersonal associations can stem from cultural norms (Karpinski \& Hilton, 2001) but also from other sources of information, including the media and other people (Olson \& Fazio, 2004; Han, Olson, \& Fazio, 2006). Although such extrapersonal associations may affect how participants solve the mapping problem posed by the IAT, they have to be distinguished from personal associations which, unlike extrapersonal associations, guide behavior once they are automatically activated (Olson \& Fazio, 2004). The implication is that findings with the alcohol-IAT indicating that alcohol is implicitly associated with negative valence could at least partly reflect negative extrapersonal knowledge about drinking alcohol that is unrelated to drinking decisions. Since information about the negative effects of substance abuse is currently abundantly available 
in our society it is plausible that the alcohol-IAT at least partly reflects negative extrapersonal knowledge rather than personally relevant associations. Negative extrapersonal information about drinking alcohol is probably readily available in drinkers' memory but does not necessarily have to converge with one's attitudes, beliefs, and associations and can be irrelevant to decision about drinking alcohol.

According to Olson and Fazio (2004), three features of the IAT procedure in particular contribute to contamination by extrapersonal associations. First, it is argued that the category labels "positive" and "negative" carry a normative implication. Second, category exemplars are typically normatively positive or negative. Finally, giving error feedback also suggests that there is a normatively correct response. Therefore, Olson and Fazio introduced the personalized IAT which reduces extrapersonal contamination by replacing the labels "positive" and "negative" with the labels "I like" and "I dislike", by using exemplars that are not normatively associated with valence but that have little evaluative consensus (e.g., football, coffee) and by refraining from giving error feedback. Thus, the personalized IAT attempts to personalize responses by allowing participants to classify stimuli without reference to normative information. The present study explores whether previous findings with the alcohol-IAT could be due to negative extrapersonal contamination rather than negative implicit alcohol associations. Alcohol-related cognitions were therefore measured with an IAT that was personalized as in Olson and Fazio (2004). It was expected that this personalized IAT would show positive rather than negative implicit associations with alcohol, supporting the hypothesis that negative alcohol associations, as previously found with the IAT, were for a large part due to extrapersonal contamination. In addition, the personalized IAT was expected to predict alcohol use and alcohol-related problems above explicit alcohol-related attitudes and expectancies.

\section{METHOD}

\section{Participants}

Forty-two male students of Maastricht University (mean age $=21$ years, $S D=4.5$ ) voluntarily participated in exchange for a gift certificate. Average alcohol consumption per week was 25.05 (SD = 13.23) Dutch standard drinks. On the 18-items version of the Rutgers Alcohol Problem Index (RAPI; White \& Labouvie, 2000), participants had an average item score of .73 (SD =.37). The average item score within clinical samples is about 80 (White \& Labouvie, 1989). On the Alcohol Use Disorder Identification Test (AUDIT; Saunders, Aasland, Babor, De la Fuente, \& Grant, 1993), participants' mean score was $12.67(S D=3.43)$, while the proposed cut-off score for the screening of alcohol-related problems is 11 . 


\section{Materials and Measures}

Alcohol Use. Alcohol use was measured with a self-report questionnaire (Wiers, Hoogeveen, Sergeant, \& Gunning, 1997) based on the timeline follow-back method (Sobell \& Sobell, 1990). Participants were asked to indicate how many drinks of different types of alcoholic beverages they consumed during each day of the past week, and how many drinks they typically consumed on each day of the week.

Alcohol-Related Problems. Alcohol-related problems were assessed with the RAPI and with the AUDIT. The RAPI described 18 alcohol-related problems or situations for which participants indicated how often they experienced these situations on a 5-point Likert scale $(0=$ never, $4=$ very often) (Cronbach's $\alpha=.74)$. The AUDIT consisted of 10 questions $(\alpha=.62)$, of which the first three were related to alcohol use, and the other seven to alcohol-related problems.

Personalized IAT. In the personalized IAT, two target categories were presented, one consisting of 5 alcoholic drinks (label "alcohol") and the other of 5 soft drinks (label "soda"). The alcohol and soda categories were matched on familiarity ( $M=5.22, S D=$ 1.29 , and $M=5.64, S D=2.12$, respectively; $1=$ completely unfamiliar, $7=$ very familiar), valence $(M=4.42, S D=.92$, and $M=4.44, S D=.88$, respectively; $1=$ very negative, $7=$ very positive), arousal $(M=4.20, S D=.80$, and $M=4.32, S D=.84$, respectively; $1=$ very passive, $7=$ very active) and number of syllables. Further, one set of 10 stimuli was presented which had to be classified into two attribute categories that were labeled "I like", and "I dislike". The attribute stimuli (familiarity: $M=4.99, S D=1.03$; arousal: $M=4.47, S D=.50$ ) were evaluated as neutral on valence but with a large standard deviation $(M=4.41$, average $S D=1.56)$, suggesting that they had little evaluative consensus (cf. Olson \& Fazio, 2004). All stimuli are listed in the Appendix.

The personalized IAT was programmed in ERTS 3.18 (Beringer, 1996) and consisted of 5 blocks. Participants first practiced the target discrimination with a right and a left response key (e.g., "alcohol" vs. "soda"). All target stimuli were presented resulting in 20 trials. In the second block, they practiced the classification of the attribute dimension (e.g., "I like" vs. "I dislike") with the same response keys. Again, all attribute stimuli were presented twice for a total of 20 trials. The third block consisted of 40 trials and this was the first combination block during which both target and attribute stimuli were presented twice (e.g., "alcohol" and "I like" vs. "soda" and "I dislike"). Next, participants received 20 trials during which they practiced the reversed attribute discrimination, followed by the reversed combination block (e.g., "alcohol" and "I dislike" vs. "soda" and "I like") which consisted of 40 trials. Target and attribute stimuli were always presented randomly in alternating order. Stimuli were presented in the middle of the computer screen, in black against a grey background. Instructions were presented before each task. During the task, 
the labels of the categories assigned to the left and right response key were presented in the corresponding upper corner of the computer screen. Stimuli remained on screen until a response was given. The intertrialinterval was 250 ms. No feedback was presented.

Explicit Expectancies and Attitudes. Explicit alcohol-related expectancies were measured with an expectancy questionnaire that consisted of 6 positive expectancy items $(\alpha=.84)$, and 6 negative expectancy items $(\alpha=.86)$. Each item asked participants to indicate on a $100 \mathrm{~mm}$ Visual Analogue Scale (VAS) how much they agreed $(0=$ completely disagree, $100=$ completely agree) with the statement: "After drinking alcohol, I feel ..." which was completed with the following words: pleasant, happy, sociable, funny, amusing, and outgoing for the positive expectancy items, miserable, sad, lonely, gloomy, unpleasant and unhappy for the negative expectancy items. Explicit attitudes toward alcohol were assessed with an attitude questionnaire which consisted of 4 semantic differentials: Participants indicated on a $100 \mathrm{~mm}$ VAS how much they considered drinking alcohol to be unpleasant-pleasant, bad-good, boring-fun, and stupid-smart. The first and third item formed an affective attitude component $(\alpha=.65)$, the other two items a cognitive attitude component $(\alpha=.68)$.

\section{Procedure}

After filling out an informed consent form, participants performed the personalized IAT. The response assignment of the target categories as well as the order of the two combination tasks was counterbalanced. Afterwards, participants filled out the expectancy questionnaire and the attitude questionnaire. Finally, participants received the alcohol use questionnaire, the AUDIT and the RAPI and they judged all IAT stimuli on familiarity, valence and arousal.

\section{RESULTS}

\section{Implicit Alcohol Associations}

IAT effects were calculated with the D600 scoring algorithm (Greenwald, Nosek, \& Banaji, 2003). Following the formula presented by Greenwald et al. (2003), practice blocks were included, error penalties $(600 \mathrm{~ms})$ were given, and results were standardized at the level of the participant. The D600 measure was calculated as the performance difference between the two combination tasks so that higher scores indicate faster performance when "alcohol" was paired with "I dislike" (vs. "soda" and "I like") than when "alcohol" and "I like" were assigned to the same response (vs. "soda" and "I dislike"). Preparatory analyses revealed no influential outliers on IAT data. Results yielded a borderline significant IAT effect, $+(41)=$ $1.96, p=.056$, indicating that participants associated alcohol more with positive valence than with negative valence. 


\section{Explicit Alcohol-Related Expectancies and Attitudes}

Mean scores were calculated separately for positive and negative expectancy items. A comparison of mean positive expectancy scores $(M=66.62, S D=10.22)$ and mean negative expectancy scores $(M=22.60, S D=10.94)$, showed that participants agreed significantly more with positive expectancies than with negative expectancies, $t(41)=16.36$, $p<.001$. Mean scores were also calculated separately for the affective and cognitive alcohol attitude component. Results showed that participants' mean affective attitude score $(M=74.11, S D=12.14)$ deviated significantly from the midpoint of the scale, $t(41)=$ $12.87, p<.001$, indicating that participants had a positive attitude towards alcohol. Mean cognitive attitude scores $(M=47.79, S D=14.17)$, however, did not deviate from the midpoint of the scale, $t(41)=-1.01, p=.317$. We then examined the relationship of the personalized IAT with alcohol-related expectancies and attitudes. The personalized IAT was significantly correlated with negative expectancies, $r=.33, p=.031$, and borderline significantly correlated with both positive expectancies, $r=-.29, p=.067$, and the affective attitude component, $r=-.30, p=.056$. The personalized IAT was uncorrelated with the cognitive attitude component, $r=.03, p=.873$.

\section{Relationship of Implicit Associations to Alcohol Use and Related Problems}

Alcohol consumption during the past week and average weekly alcohol consumption were estimated from the alcohol use questionnaire. These two estimates were then log-transformed and the mean of these two estimates was calculated as a measure of alcohol use. An estimate for mean alcohol-related problems was computed as the mean of the z-transformed RAPI and AUDIT log-transformed sum scores. These two estimates were significantly correlated, $r=.49$, $p=.001$, indicating that participants with increased levels of alcohol consumption also experienced more alcohol-related problems. The personalized IAT correlated significantly with both alcohol use, $r=-.43, p=.005$, and alcohol-related problems, $r=-.39, p=.012$.

Next, we tested the predictive validity of the explicit measures and the personalized IAT. The hierarchical regression analysis predicting alcohol use is shown in Table 1. Inspection of the Cook's distances showed one influential case. This participant was therefore excluded from the hierarchical regression analysis. Explicit alcohol-related expectancies and attitudes were entered in step 1 of the regression analysis while the personalized IAT was entered in step 2. In step 1, only the affective attitude component significantly predicted alcohol use. In step 2, the personalized IAT was found to significantly predict alcohol use above the variance explained by the affective attitude component. The same procedure was also followed for the prediction of alcohol-related problems (see Table 2). None of the explicit measures entered in step 1 significantly predicted alcohol-related problems whereas entering the personalized IAT in step 2 significantly increased the variance explained. Results demonstrated that only the personalized IAT significantly predicted alcohol-related problems. 


\section{CHAPTER 4}

Table 1

Summary of hierarchical regression analysis for the prediction of self-reported alcohol use by explicit alcohol-related cognitions and effect scores ${ }^{a}$ of the personalized IAT.

\begin{tabular}{llcccccc}
\hline Step & Variable & $B$ & SE B & $\beta$ & SE $\beta$ & $\dagger$ & $p$ \\
\hline 1 & Positive alcohol expectancies & -.02 & .01 & -.37 & .22 & -1.65 & .109 \\
& Negative alcohol expectancies & -.00 & .01 & .05 & .17 & .27 & .785 \\
& Affective attitude component & .03 & .01 & .62 & .22 & 2.83 & .008 \\
& Cognitive attitude component & -.00 & .01 & -.03 & .17 & -.18 & .858 \\
& Positive alcohol expectancies & -.02 & .01 & -.36 & .21 & -1.72 & .095 \\
& Negative alcohol expectancies & .01 & .01 & .12 & .17 & .70 & .490 \\
Affective attitude component & .03 & .01 & .51 & .21 & 2.39 & .022 \\
Cognitive attitude component & -.00 & .01 & -.03 & .16 & -.18 & .861 \\
Personalized IAT & -.38 & .17 & -.36 & .16 & -2.28 & .029
\end{tabular}

Note. $F(4,36)=2.09, p=.103, R^{2}=.19$, for step $1 ; F_{\text {change }}(1,35)=5.18, p=.029, R_{\text {change }}^{2}=.11$, for step 2 . Final model: $R^{2}=.29, R^{2}{ }_{\text {odjusted }}=.19, F(5,35)=2.90, p=.027 ;{ }^{\circ}$ Compatibility effects in the personalized IAT were scored so that positive values indicate faster responses if "alcohol" was paired with "I dislike" and "soda" with "I like".

Table 2

Summary of hierarchical regression analysis for the prediction of self-reported alcohol-related problems by explicit alcohol-related cognitions and effect scores ${ }^{a}$ of the personalized IAT.

\begin{tabular}{llcccccc}
\hline Step & Variable & $B$ & SE B & $\beta$ & SE $\beta$ & $\dagger$ & $p$ \\
\hline 1 & Positive alcohol expectancies & -.02 & .02 & -.21 & .21 & -.97 & .337 \\
& Negative alcohol expectancies & -.01 & .01 & -.16 & .17 & -.92 & .366 \\
& Affective attitude component & .03 & .01 & .38 & .20 & 1.95 & .059 \\
& Cognitive attitude component & -.01 & .01 & -.09 & .17 & -.52 & .606 \\
& Positive alcohol expectancies & -.02 & .02 & -.24 & .21 & -1.15 & .256 \\
$\quad$ Negative alcohol expectancies & -.01 & .01 & -.07 & .17 & -.44 & .666 \\
Affective attitude component & .02 & .01 & .33 & .19 & 1.73 & .092 \\
Cognitive attitude component & -.01 & .01 & -.08 & .16 & -.49 & .630 \\
Personalized IAT & -.51 & .25 & -.33 & .16 & -2.05 & .047
\end{tabular}

Note. $F(4,37)=1.37, p=.264, R^{2}=.13$, for step $1 ; F_{\text {change }}(1,36)=4.21, p=.047, R_{\text {change }}^{2}=.09$, for step 2 . Final model: $R^{2}=.22, R_{\text {odjusted }}^{2}=.11, F(5,36)=2.03, p=.098 ;{ }^{\circ}$ Compatibility effects in the personalized IAT were scored so that positive values indicate faster responses if "alcohol" was paired with "I dislike" and "soda" with "I like". 


\section{DISCUSSION}

Previous studies that used the IAT to examine implicit alcohol-related cognitions repeatedly demonstrated negative associations with alcohol, regardless of level of alcohol consumption (e.g., De Houwer et al., 2004; Wiers et al., 2002, 2005). Recent research, however, suggests that the IAT does not necessarily reflect implicit associations but can also be affected by extrapersonal knowledge (e.g., Han et al., 2006; Olson \& Fazio, 2004). Hence, previous findings with the alcohol-IAT could at least partly reflect negative extrapersonal information about drinking alcohol that is attitude-irrelevant. Therefore, in the present study, implicit alcohol associations were measured with a personalized alcohol-IAT designed to eliminate extrapersonal contamination in the IAT (cf. Olson \& Fazio, 2004). The personalized IAT revealed positive implicit associations with alcohol that predicted alcohol-related behavior above explicit attitudes toward drinking alcohol. Hence, these results suggest that previous evidence for negative implicit alcohol associations was at least partly caused by behavior-irrelevant extrapersonal contamination in the alcoholIAT and that behavior-relevant implicit alcohol associations are positive rather than negative.

The personalized IAT yielded a significant IAT effect, indicating that alcohol was associated more with positive than with negative valence. In addition, these alcohol associations were related to and predicted self-reported alcohol consumption and alcoholrelated problems. These results are in line with the idea that the traditional IAT is contaminated by negative extrapersonal knowledge about drinking alcohol and that this contamination is reduced in the personalized IAT. These results are also consistent with studies that have assessed implicit alcohol-related cognitions in a unipolar format. More specifically, when alcohol associations were no longer tested in a bipolar format (e.g., positive vs. negative) as is typically done in IAT research, but in a unipolar format (e.g., positive vs. neutral and negative vs. neutral), it became evident that alcohol is not only implicitly associated with negative valence, but also with positive valence (Houben \& Wiers, 2006c, 2006d; Jajodia \& Earleywine, 2003). Importantly, these studies also showed that positive implicit alcohol associations were at least moderately related to alcohol-related behavior whereas negative implicit alcohol associations were not. Together, these results suggest that alcohol could be more easily paired with a negative attribute category than with a positive attribute category (in a bipolar IAT) or with a neutral attribute category (in the unipolar IAT) because of negative extrapersonal knowledge that does not influence drinking behavior. Importantly, when such extrapersonal contamination is reduced in the bipolar IAT, for example by personalizing the IAT, or when implicit alcohol associations are tested in a unipolar format, it becomes clear that implicit associations with alcohol are positive (or possibly ambivalent) and that positive implicit alcohol associations are meaningfully related to drinking behavior. 
Finally, it is important to note that De Houwer et al. (2004) also personalized the IAT by using the attribute labels "liked" and "disliked" and by using individually selected liked and disliked attribute stimuli, but still found support for negative associations with alcohol (in alcoholics). However, the IAT used by De Houwer et al. (2004) differs from the personalized IAT used in this study as well as from the personalized IAT as developed by Olson and Fazio (2004) in two aspects: First, in the IAT used by De Houwer et al. (2004) presented attribute stimuli that were individually selected instead of idiosyncratic attribute stimuli which were the same for all participants. Second, De Houwer et al. (2004) did not use attribute category labels that unambiguously directed participants' focus to their own evaluation of the target concepts. Importantly, Han et al. (2006) demonstrated that primarily the use of such personalized labels reduces extrapersonal contamination in the IAT. Hence, it could be argued that the IAT in the study by De Houwer et al. (2004) was probably still susceptible to extrapersonal contamination, which could explain why they were unable to find support for positive associations with alcohol. Alternatively, negative experiences following alcohol use may have caused stronger negative associations in alcoholics in treatment (Wiers, Houben, Smulders, Conrod, \& Jones, 2006, cf. Jones \& McMahon, 1996).

In conclusion, the present study not only increases our understanding of automatic cognitive processes that are involved in alcohol use and misuse, but also carries implications with respect to interventions. Specifically, the present results suggest that it might be useful for future interventions to simultaneously target both explicit and implicit alcohol-related cognitions. Current interventions are typically aimed at changing explicit alcohol-related cognitions, making people aware of the negative consequences of their addictive behavior and devaluating the positive consequences. However, if implicit alcohol-related cognitions, and more specifically implicit positive associations with alcohol, are not also subject to change during such interventions, they will continue to automatically steer the addictive behavior in the future. It is therefore interesting for future research to search for new ways to reduce the strength or the impact of positive implicit alcohol associations as well as to examine whether personally relevant implicit negative associations with alcohol can be created that reduce consumption. 


\section{APPENDIX}

Target Stimuli

Alcohol: beer, wine, whisky, drink, vodka

Soda: Fanta, Coca Cola, Sprite, sinas (lemonade), ice-tea

Attribute Stimuli

Coffee, spinach, garlic, art, soccer, jogging, secret, cleaning, disco, museum 


$$
\text { D) }
$$




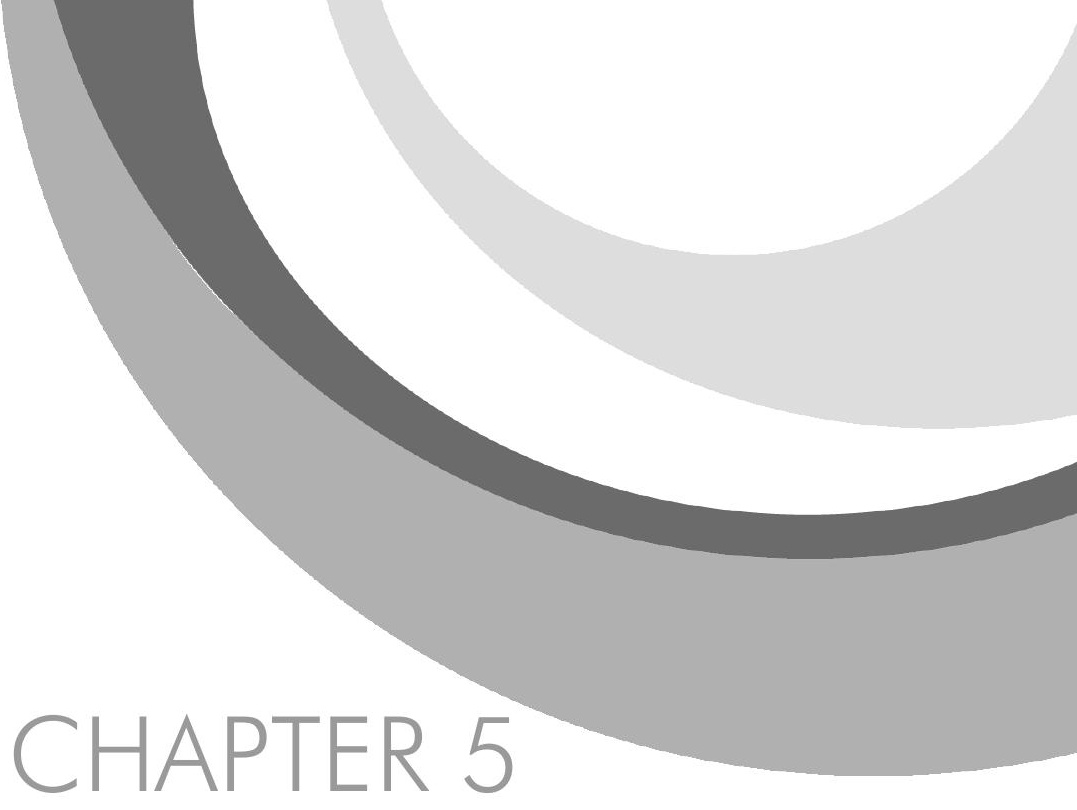

\section{Personalizing the Alcohol-IAT with Individualized Stimuli: Relationship with Drinking Behavior and Drinking-Related Problems}

Adapted version of: Houben, K., \& Wiers, R. W. (2006). Personalizing the Alcohol-IAT with Individualized Stimuli: Relationship with Drinking Behavior and Drinking-Related Problems. Accepted for publication pending revisions. 


\begin{abstract}
Research aimed at uncovering implicit cognitive processes involved in alcohol use and abuse has demonstrated that implicit attitudes toward alcohol are negative and unrelated to drinking behavior. Here, it was examined whether these findings could be due to contamination of the IAT by extrapersonal associations that are irrelevant to behavior. Participants performed a traditional alcohol-IAT as well as a personalized IAT, which has been demonstrated to reduce extrapersonal contamination. Additionally, the personalized IAT presented individualized stimuli, which should further reduce extrapersonal contamination. Consistent with this hypothesis, significantly weaker IAT effects emerged in the personalized IAT compared to the traditional IAT. However, both the traditional and personalized IAT still indicated implicit negative attitudes toward alcohol. Incremental predictive validity was demonstrated for both tasks. Importantly, these findings underscore the importance of implicit attitudes toward alcohol as determinants of alcohol use and abuse.
\end{abstract}

\title{
INTRODUCTION
}

For many years, psychologists have been interested in the role of alcohol-related cognitions in alcohol use and abuse. Most of this research has been conducted with selfreport measures of alcohol-related cognitions. This may be an important limitation since self-report measures are susceptible to self-presentation and because self-report measures might not be well suited to index automatic processes that play a role in alcohol use and abuse. Therefore, researchers have recently started using indirect measures of alcoholrelated cognitions which are more resistant to self-presentation biases and which might reveal implicit cognitive processes that cannot be easily assessed with self-report measures. Implicit cognitions can be defined as cognitions that are activated automatically and that can influence behavior automatically, outside conscious awareness (Gawronski, Hofmann, \& Wilbur, 2006; De Houwer \& Moors, in press). As such, indirect measures could lead to important new insights in the study of alcohol-related cognitions involved in alcohol use and abuse.

In order to reveal implicit cognitive processes that influence alcohol use and abuse, Wiers, van Woerden, Smulders, and de Jong (2002) used the Implicit Association Test (IAT, Greenwald, McGhee, \& Schwartz, 1998) as an indirect measure of implicit alcoholrelated cognitions in light and heavy drinkers. In the IAT, the participants' task is to quickly and accurately classify stimuli into two target categories and two attribute categories using two response keys. The underlying logic is that classification performance should be better 
when associated target concepts and attribute categories share a response (i.e., compatible response assignment) than when target categories are paired with unassociated attribute categories (i.e., incompatible response assignment). Using the IAT, Wiers et al. found that both light and heavy drinkers were faster when alcohol and negative attributes were assigned to the same response, and soda and positive attributes to the other response than when alcohol shared a response with positive attributes and soda with negative attributes. Hence, these results indicate that both light and heavy drinkers had implicit negative evaluative associations with alcohol, or implicit negative attitudes toward alcohol. Wiers et al. did find a differentiation between light and heavy drinkers with a second IAT: Heavy drinkers were faster when alcohol shared a response with arousal and soda with sedation, than when alcohol and sedation were assigned to one response and soda and arousal to the other. This effect, however, was absent in light drinkers. Wiers et al. hypothesized that these results were in line with the incentivesensitization theory of Robinson and Berridge (1993) according to which addictive behaviors such as alcohol use are related more to "wanting" (i.e., sensitized arousal) the addictive substance than to "liking" of the substance. These results were also replicated in a sample of heavy drinkers (Wiers, van de Luitgaarden, van den Wildenberg, \& Smulders, 2005) as well as in a sample of patients undergoing treatment (De Houwer, Crombez, Koster, \& De Beul, 2004). Thus, these findings suggest that implicit alcohol associations with arousal may exert an automatic influence on alcohol use and abuse, while implicit attitudes toward alcohol do not appear to be involved in alcohol-related behavior.

It is, however, possible that results with the alcohol-IAT do not reflect implicit negative attitudes toward alcohol but instead culturally shared associative knowledge about drinking alcohol and its negative consequences, which does not influence behavior. In line with such a conclusion, it was demonstrated that the IAT is sensitive to so-called extrapersonal associations that do not form the basis of one's attitudes and that are irrelevant to behavior. Such extrapersonal associations may stem from cultural norms (Karpinski \& Hilton, 2001) or from other sources of information, such as the media and other people (Olson \& Fazio, 2004). Although such extrapersonal associations may affect IAT performance, they need to be distinguished from personal associations that, unlike extrapersonal associations, are automatically activated and guide behavior (Olson \& Fazio, 2004). Olson and Fazio (2004) argue that contamination of the IAT by extrapersonal associations is promoted due to normative implications of the attribute labels 'positive' and 'negative' as well as the use of normatively positive and negative attribute exemplars. In addition, giving error feedback in the IAT also suggests that there is a normatively correct answer. Therefore, they developed the personalized IAT which reduces contamination by extrapersonal associations because all references to normative information are eliminated: The labels 'positive' and 'negative' are replaced with the labels 'I like' and 'I dislike', stimuli are used that are not normatively associated with valence but that have little evaluative consensus (e.g., football, coffee), and no error 
feedback is presented. Houben and Wiers (2006b) recently examined whether the alcoholIAT is affected by extrapersonal contamination using the personalized IAT as developed by Olson and Fazio (2004). In line with the hypothesis, the personalized IAT revealed implicit positive attitudes toward alcohol and was strongly correlated with alcohol use and alcoholrelated problems, suggesting a reduced influence of extrapersonal associations.

However, as has also been pointed out by Fadardi, Cox, and Klinger (2006), the personalized IAT presents stimulus words for which there is no evaluative consensus, but these stimuli are nevertheless the same for all participants. It is questionable how well an IAT that presents standard stimuli can capture participants' personal associations, since standard stimuli will probably differ in the degree to which they are both known and liked across participants. This is particularly true when applied to alcohol research since there is probably great variation in the alcoholic drinks that are known and liked by participants. In line with this idea, Houben and Wiers (2006e) have demonstrated that implicit attitudes toward beer, in contrast to more general implicit attitudes toward alcohol, were related to alcohol consumption in a sample of participants who drank beer on a regular basis. Hence, it might be useful to personalize the IAT, not only by removing all reference to normative information, but also by using individualized words (e.g., participants' favorite drinks). This possibility was examined in the present study. Participants' scores on a standard alcohol-IAT, similar to those used in previous studies, were compared to their scores on a personalized IAT that presented stimuli that were individualized for each participant. More specifically, each participant reported his or her favorite alcoholic drinks and sodas, which were then used as the stimuli for the target categories in the personalized IAT that was performed by that participant. The target categories were labeled 'my drink' and 'my soda', respectively. Further, all participants also listed positive and negative words that were subsequently used as the stimuli for the attribute categories ' like' and 'I dislike', respectively. Finally, no error feedback was presented during the personalized IAT. It was expected that the standard IAT would show support for implicit negative attitudes toward alcohol, replicating previous findings. In contrast, it was expected that the personalized IAT would yield evidence for implicit positive attitudes toward alcohol. Importantly, it was expected that the personalized IAT would show a stronger relation to self-reported alcohol use and alcohol-related problems compared to the standard IAT, suggesting a reduced contamination by extrapersonal associations in the personalized IAT. 


\section{METHOD}

\section{Participants}

Forty-six students of Maastricht University (31 females, 15 males; mean age $=21.33$ years, $S D=1.84$ ) voluntarily participated in exchange for a gift certificate. Average alcohol consumption per week was $15.63(S D=14.49)$ Dutch standard drinks ${ }^{7}$. On the 18-items version of the Rutgers Alcohol Problem Index (RAPI; White \& Labouvie, 2000), participants' mean item score was $.51(S D=.38)$ while the average item score in clinical samples is about .80 (White \& Labouvie, 1989). On the Alcohol Use Disorder Identification Test (AUDIT; Saunders, Aasland, Babor, De la Fuente, \& Grant, 1993), participants' mean score was $11.11(S D=5.38)$, while the proposed AUDIT cut-off score for the screening of alcohol-related problems is 11 (Saunders et al., 1993).

\section{Materials and Measures}

Alcohol Use. Alcohol use was measured with a self-report questionnaire (Wiers, Hoogeveen, Sergeant \& Gunning, 1997) based on the timeline follow-back method (Sobell \& Sobell, 1990). Participants were asked to indicate how many drinks of different types of alcoholic beverages they consumed during each day of the past week, and how many drinks they typically consumed on each day of the week.

Alcohol-Related Problems. Alcohol-related problems were assessed with the RAPI and with the AUDIT. The RAPI described 18 alcohol-related problems or situations for which participants indicated how often they experienced these situations on a 5-point Likert scale $(0=$ never, $4=$ very often) (Cronbach's $\alpha=.82)$. The AUDIT consisted of 10 questions $(\alpha=.85)$, of which the first three were related to alcohol use, and the other seven to alcohol-related problems.

Implicit Association Test. In the traditional IAT, two target categories were presented, one consisting of 5 alcoholic drinks (label "alcohol") and the other of 5 soft drinks (label "soda"). The alcohol and soda categories were matched on familiarity $(M=$ $5.20, S D=1.64$, and $M=5.67, S D=1.35$, respectively; $1=$ completely unfamiliar, 7 = very familiar), valence $(M=4.08, S D=1.15$, and $M=4.43, S D=.96$, respectively; $1=$ extremely negative, $7=$ extremely positive), arousal $(M=3.84, S D=.97$, and $M=$ $4.25, S D=1.02$, respectively; $1=$ extremely passive, $7=$ extremely active), and number of syllables. Further, in the traditional IAT, the two attribute categories consisted of 5 positive (label "pleasant"; $M=6.45, S D=.61$ ), and 5 negative (label "unpleasant"; $M=$

\footnotetext{
${ }^{7}$ A standard alcoholic drink in Holland contains less alcohol than a standard English or American alcoholic drink: 10 vs. $14 \mathrm{~g}$, respectively.
} 
$1.43, S D=.56)$ stimuli. The attribute categories were matched, as closely as possible, on familiarity $(M=6.21, S D=.88$, and $M=5.29, S D=1.64$, respectively), arousal $(M=$ $4.22, S D=1.10$, and $M=3.84, S D=.84$, respectively), and number of syllables. All stimuli that were presented in the traditional IAT are listed in the Appendix. For the personalized IAT, all stimuli were selected through a questionnaire completed by the participants before the onset of the study. On this questionnaire, participants listed their five most favorite alcoholic drinks as well as their five most favorite sodas, which were subsequently used as stimuli for the alcohol and soda target categories, respectively. Also, participants listed five things they associated with positive feelings, and five things they associated with negative feelings. These positive and negative stimuli were then used as stimuli for the positive and negative attribute categories, respectively. The target categories were labeled 'my drink' (alcohol) and 'my soda' (soda), while the attribute categories were labeled 'I like' (positive) and 'I dislike' (negative).

The traditional IAT and the personalized IAT were programmed in ERTS 3.18 (Beringer, 1996) and consisted of 5 blocks. Participants first practiced the target discrimination with a right and a left response key (e.g., 'alcohol' vs. 'soda'). All target stimuli were presented twice, resulting in 20 target practice trials. In the second block, participants received 20 trials during which they practiced the classification of the attribute dimension (e.g., 'unpleasant' vs. 'pleasant' or 'I dislike' vs. 'I like') with the same response keys. Again, all attribute stimuli were presented twice. The third block was the first combination block during which both target and attribute stimuli were presented twice, resulting in 40 trials (e.g., 'alcohol' + 'unpleasant'/'I dislike' vs. 'soda' + 'pleasant'/'I like'). Next, participants received 20 trials in which they practiced the reversed attribute discrimination (e.g., 'pleasant' vs. 'unpleasant' or 'I like' vs. 'I dislike'), followed by the reversed combination block, which consisted of 40 trials (e.g., 'alcohol' + 'pleasant'/'I like' vs. 'soda' + 'unpleasant'/'l dislike').

Target and attribute stimuli were always presented randomly in alternating order. Stimuli were presented in the middle of the computer screen, in black against a grey background. Instructions were presented before each task. During the task, the labels of the categories assigned to the left and right response key were presented in the corresponding upper corner of the computer screen. Stimuli remained on screen until a response was given. The intertrialinterval was $250 \mathrm{~ms}$. During the traditional IAT, feedback was presented in red beneath the stimuli after an incorrect response ('wrong'), and when responses were too fast (<300 ms; 'too fast') or too slow (> $3000 \mathrm{~ms}$; 'too slow'). No feedback was presented during the personalized IAT.

Explicit Expectancies and Attitudes. Explicit alcohol-related expectancies were measured with an expectancy questionnaire that consisted of 6 positive expectancy items $(\alpha=.83)$, and 6 negative expectancy items $(\alpha=.84)$. Each item asked participants to indicate on a $100 \mathrm{~mm}$ Visual Analogue Scale (VAS) how much they agreed $(0=$ 
completely disagree, $100=$ completely agree) with the statement: "After drinking alcohol, I feel. .." which was completed with the following words: active, lively, impulsive, spontaneous, talkative, and sociable for the positive expectancy items, miserable, sad, lonely, unhappy, unpleasant, and gloomy for the negative expectancy items. Explicit attitudes toward alcohol were assessed with an attitude questionnaire which consisted of 2 semantic differentials: Participants indicated on a $100 \mathrm{~mm}$ VAS how much they considered drinking alcohol to be unpleasant-pleasant, boring-fun $(\alpha=.92)$.

\section{Procedure}

First, participants filled out an informed consent form. Participants then performed the traditional IAT and the personalized IAT in balanced order. Also, the response assignment of the target categories, as well as the order of the combination tasks were balanced across participants and remained the same during both the traditional IAT and the personalized IAT. Next, participants filled out the alcohol expectancy questionnaire, the alcohol attitude questionnaire, the alcohol use questionnaire, the AUDIT and the RAPI. Finally, participants rated all stimuli on familiarity, valence and arousal.

\section{RESULTS}

\section{Implicit Alcohol Associations}

IAT effects were calculated with both the D600 scoring algorithm (Greenwald, Nosek, \& Banaji, 2003) and with the conventional log measure (Greenwald et al., 1998) because the two algorithms can show markedly different results (e.g., Wiers et al., 2005) and because there is no consensus yet on which measure is optimal (e.g., Greenwald, Nosek, Banaji, \& Klaver, 2005 vs. Rothermund, Wentura, \& De Houwer, 2005). Therefore, results with the D600 measure are reported and where differences were found with the conventional measure, these will be indicated. First, trials with response latencies below $300 \mathrm{~ms}$ or above $3000 \mathrm{~ms}$ were recoded to $300 \mathrm{~ms}$ and $3000 \mathrm{~ms}$, respectively. Mean response latencies were calculated for the two combination tasks separately for the traditional IAT and the personalized IAT. Next, the conventional measure was calculated as the difference between these two log-transformed means so that higher IAT scores indicate faster performance for the compatible response assignment ('alcohol' + 'unpleasant'/'I dislike' vs. 'soda' + 'pleasant'/l' like') than for the incompatible response assignment ('alcohol' + 'pleasant'/'I like' vs. 'soda' + 'unpleasant'/'I dislike'). The D600 measure was calculated in the same direction. Further, following the formula presented by Greenwald et al., practice blocks were included, error penalties $(600 \mathrm{~ms})$ were given, and results were standardized at the level of the participant. 
Preparatory analyses revealed no influential outliers on IAT data. Results revealed a significant difference between the traditional IAT and the personalized IAT, $t(45)=2.20, p$ $=.033^{8}$. Consistent with previous findings, the IAT effect in the traditional IAT was significant, $t(45)=6.61, p<.001$, indicating that participants were faster when alcohol shared a response with the negative attribute category than when alcohol was paired with the positive attribute category. In the personalized IAT, the IAT effect was also significant, $t(45)=3.26, p=.002$, and in the same direction as the IAT effect in the traditional IAT. Hence, results demonstrate that participants associated alcohol more with negative than with positive in the traditional IAT, and to a lesser extent also in the personalized IAT.

\section{Explicit Alcohol-Related Expectancies and Attitudes}

Mean scores were calculated separately for the positive and negative expectancy items. Results showed that participants agreed significantly more with positive expectancies $(M=$ $65.02, S D=16.80)$ than with negative expectancies $(M=18.70, S D=12.41), t(45)=$ $13.66, p<.001$. Further, mean attitude scores were also calculated from the alcohol attitude questionnaire. Results showed that participants' mean attitude score $(M=72.25$, $S D=18.44)$ deviated significantly from the midpoint of the scale, $t(45)=8.18, p<$ .001 , indicating that participants had a favorable attitude towards alcohol. We then examined the relationship of the traditional IAT and the personalized IAT with alcoholrelated expectancies and attitudes (see Table 1). None of the correlations between the two IAT versions and explicit alcohol-related cognitions reached conventional levels of significance.

\section{Relationship of Implicit Associations to Alcohol Use and Related Problems}

Alcohol consumption during the past week and average weekly alcohol consumption were estimated from the alcohol use questionnaire. These two estimates were then logtransformed and the mean of these two estimates was calculated as a measure of alcohol use. An estimate for mean alcohol-related problems was computed as the mean of the ztransformed RAPI and AUDIT log-transformed sum scores. These two estimates were significantly correlated, $r=.74, p<.001$, indicating that participants with increased levels of alcohol consumption also experienced more alcohol-related problems. As can be seen in Table 1, the traditional IAT and the personalized IAT both correlated significantly with alcohol use.

\footnotetext{
${ }^{8}$ When results were analyzed using the conventional IAT effect measure, the difference between the traditional IAT and the personalized IAT was borderline significant, $t(45)=1.70, p=.097$, but in the same direction.
} 
Table 1

Correlations of both the traditional IAT and the personalized IAT with explicit alcohol-related expectancies and attitudes, alcohol use, and alcohol-related problems.

\begin{tabular}{|c|c|c|c|c|c|}
\hline \multirow[b]{2}{*}{ Variable } & \multicolumn{2}{|c|}{ IAT } & \multicolumn{2}{|c|}{ Expectancies } & \multirow[t]{2}{*}{ Attitude } \\
\hline & Traditional & Personalized & Positive & Negative & \\
\hline Personalized IAT & $.39^{* *}$ & - & - & - & - \\
\hline Positive expectancies & -19 & .09 & - & - & - \\
\hline Negative expectancies & -.15 & -.11 & -.22 & - & - \\
\hline Attitude toward alcohol & -.10 & -.17 & $.47^{* *}$ & $-.55^{* *}$ & - \\
\hline Alcohol use & $-.37^{*}$ & $-.31^{*}$ & $.33^{*}$ & -.17 & $.48^{* *}$ \\
\hline Alcohol-related problems & -.23 & -.24 & $.44^{*}$ & -.17 & $.46^{* *}$ \\
\hline
\end{tabular}

Next, we tested the predictive validity of the explicit measures and the incremental validity of the traditional IAT and the personalized IAT using hierarchical regression analysis. Regression analyses were performed separately for the prediction of alcohol use and alcohol-related problems. Explicit alcohol-related expectancies and attitudes toward alcohol were always entered in step 1 . In order to examine the incremental validity of both the traditional IAT and the personalized IAT, regression analyses were performed twice, once with the traditional IAT entered in step 2 and the personalized IAT entered in step 3, and once with the two IAT versions entered in the reversed order. The hierarchical regression analysis predicting alcohol use is shown in Table 2. In step 1 of the regression analysis, attitudes toward alcohol significantly predicted alcohol use. When the traditional IAT was entered in step 2, results showed that the traditional IAT significantly predicted alcohol use above explicit attitudes toward alcohol. However, when the personalized IAT was then entered in step 3, the variance explained was not significantly increased. When the personalized IAT was entered in step 2 and the traditional IAT in step 3, results showed that the personalized IAT predicted alcohol use at borderline significance above explicit attitudes toward alcohol in step 2 while entering the traditional IAT in step 3 did not increase the variance explained ${ }^{9}$. The hierarchical regression analysis for the prediction of alcohol-related problems is shown in Table 3. In step 1, explicit positive expectancies

\footnotetext{
${ }^{9}$ When IAT effects were calculated using the conventional IAT effect measure, entering the personalized IAT in step 2 of the hierarchical regression analysis for the prediction of alcohol use significantly increased the variance explained, $F(1,41)=5.88, p=.020, R_{\text {change }}^{2}=.09$, and the personalized IAT significantly predicted alcohol use above explicit attitudes toward alcohol, $\beta=-.34, p=.020$. Entering the traditional IAT in step 3 did not increase the variance explained, $F(1,40)=1.41, p=.242$.
} 
predicted alcohol-related problems at borderline significance, while explicit attitudes toward alcohol significantly predicted alcohol-related problems. Neither the traditional IAT nor the personalized IAT significantly predicted alcohol-related problems above explicit alcohol-related expectancies and attitudes ${ }^{10}$.

Table 2

Summary of hierarchical regression analysis for the prediction of alcohol use by alcohol-related expectancies and attitudes (step 1), and IAT effect scores of the traditional IAT and the personalized IAT (step 2 and 3).

\begin{tabular}{|c|c|c|c|c|c|c|c|}
\hline Step & Variable & $B$ & SE B & $\beta$ & SE $\beta$ & $t$ & $p$ \\
\hline \multirow[t]{3}{*}{1} & Positive alcohol expectancies & .01 & .01 & .13 & .15 & .87 & .391 \\
\hline & Negative alcohol expectancies & .01 & .01 & .13 & .16 & .78 & .439 \\
\hline & Attitude toward alcohol & .02 & .01 & .48 & .18 & 2.74 & .009 \\
\hline \multirow[t]{4}{*}{2} & Positive alcohol expectancies & .01 & .01 & .08 & .15 & .56 & .581 \\
\hline & Negative alcohol expectancies & .00 & .01 & .04 & .16 & .24 & .811 \\
\hline & Attitude toward alcohol & .02 & .01 & .43 & .17 & 2.51 & .016 \\
\hline & Traditional IAT & -.46 & .20 & -.30 & .13 & -2.26 & .029 \\
\hline \multirow[t]{4}{*}{2} & Positive alcohol expectancies & .01 & .01 & .19 & .15 & 1.27 & .212 \\
\hline & Negative alcohol expectancies & .00 & .01 & .05 & .16 & .30 & .770 \\
\hline & Attitude toward alcohol & .02 & .01 & .37 & .18 & 2.02 & .050 \\
\hline & Personalized IAT & -.33 & .18 & -.26 & .14 & -1.88 & .068 \\
\hline \multirow[t]{5}{*}{3} & Positive alcohol expectancies & .01 & .01 & .13 & .15 & .85 & .399 \\
\hline & Negative alcohol expectancies & .00 & .01 & .01 & .16 & .04 & .969 \\
\hline & Attitude toward alcohol & .02 & .01 & .37 & .18 & 2.05 & .047 \\
\hline & Traditional IAT & -.36 & .22 & -.24 & .14 & -1.66 & .104 \\
\hline & Personalized IAT & -.21 & .19 & -.17 & .15 & -1.13 & .265 \\
\hline
\end{tabular}

Note. IAT effects for both the traditional IAT and the personalized IAT were scored so that positive values indicate faster responses if the alcohol category was assigned to the same response as the negative attribute category (and soda to the same response as the positive attribute category). $F(3,42)=4.74, p=.006, R^{2}=.25$, for step 1 ; When the traditional IAT was entered in step 2 and the personalized IAT in step 3, $F(1,41)=5.12, p=.029$, $R_{\text {change }}^{2}=.08$, for step 2 and $F(1,40)=1.28, p=.265, R_{\text {change }}^{2}=.02$, for step 3 . Final model (step 2$): R^{2}=.34$, $R_{\text {odjusted }}^{2}=.27, F(4,41)=5.18, p=.002$. When the personalized IAT was entered in step 2 and the traditional IAT in step $3, F(1,41)=3.52, p=.068, R_{\text {change }}^{2}=.06$, for step 2 and $F(1,40)=2.76, p=.104, R_{\text {change }}^{2}=.04$, for step 3. Final model (step 2): $R^{2}=.31, R_{\text {adiusted }}^{2}=.25, F(4,41)=4.65, p=.003$.

${ }^{10}$ When IAT effects were calculated using the conventional IAT effect measure, entering the personalized IAT in step 2 of the hierarchical regression analysis for the prediction of alcohol-related problems borderline significantly increased the variance explained, $F(1,41)=3.50, p=.069, R_{\text {change }}^{2}=.06$, and the personalized IAT predicted alcohol-related problems at borderline significance above explicit attitudes toward alcohol, $\beta=-.26, p=.069$. Entering the traditional IAT in step 3 did not increase the variance explained, $F(1,40)=.06, p=.812$. 
Table 3

Summary of hierarchical regression analysis for the prediction of alcohol-related problems by alcohol-related expectancies and attitudes (step 1), and IAT effect scores of the traditional IAT and the personalized IAT (step 2 and 3).

\begin{tabular}{|c|c|c|c|c|c|c|c|}
\hline Step & Variable & $B$ & SE B & $\beta$ & SE $\beta$ & $t$ & $p$ \\
\hline \multirow[t]{3}{*}{1} & Positive alcohol expectancies & .02 & .01 & .28 & .15 & 1.88 & .068 \\
\hline & Negative alcohol expectancies & .01 & .01 & .10 & .16 & .61 & .548 \\
\hline & Attitude toward alcohol & .02 & .01 & .38 & .17 & 2.18 & .035 \\
\hline \multirow[t]{4}{*}{2} & Positive alcohol expectancies & .01 & .01 & .26 & .15 & 1.70 & .096 \\
\hline & Negative alcohol expectancies & .00 & .01 & .06 & .16 & .345 & .732 \\
\hline & Attitude toward alcohol & .02 & .01 & .35 & .18 & 2.01 & .051 \\
\hline & Traditional IAT & -.18 & .18 & -.14 & .14 & -.99 & .328 \\
\hline \multirow[t]{4}{*}{2} & Positive alcohol expectancies & .02 & .01 & .33 & .15 & 2.20 & .034 \\
\hline & Negative alcohol expectancies & .00 & .01 & .03 & .16 & .19 & .851 \\
\hline & Attitude toward alcohol & .01 & .01 & .28 & .18 & 1.56 & .128 \\
\hline & Personalized IAT & -.24 & .15 & -.22 & .14 & -1.58 & .122 \\
\hline \multirow[t]{5}{*}{3} & Positive alcohol expectancies & .02 & .01 & .31 & .16 & 2.01 & .051 \\
\hline & Negative alcohol expectancies & .00 & .01 & .02 & .16 & .12 & .907 \\
\hline & Attitude toward alcohol & .01 & .01 & .28 & .18 & 1.54 & .133 \\
\hline & Traditional IAT & -.09 & .20 & -.06 & .15 & -.43 & .670 \\
\hline & Personalized IAT & -.22 & .17 & -.19 & .15 & -1.28 & .208 \\
\hline
\end{tabular}

Note. IAT effects for both the traditional IAT and the personalized IAT were scored so that positive values indicate faster responses if the alcohol category was assigned to the same response as the negative attribute category (and soda to the same response as the positive attribute category). $F(3,42)=5.40, p=.003, R^{2}=.28$, for step 1 ; When the traditional IAT was entered in step 2 and the personalized IAT in step $3, F(1,41)=.98, p=.328, R_{\text {change }}^{2}$ $=.02$, for step 2 and $F(1,40)=1.64, p=.208, R_{\text {change }}^{2}=.03$, for step 3 . When the personalized IAT was entered in step 2 and the traditional IAT in step $3, F(1,41)=2.50, p=.122, R_{\text {change }}^{2}=.04$, for step 2 and $F(1,40)=.18$, $p=.670, R_{\text {change }}^{2}=.00$, for step 3. Final model (step 1): $R^{2}=.28, R_{\text {odjusted }}^{2}=.23, F(3,42)=5.40, p=.003$.

\section{DISCUSSION}

Alcohol-IAT studies have repeatedly demonstrated the counterintuitive finding that drinkers display implicit negative attitudes toward alcohol that do not have a strong impact on alcohol-related behavior (e.g., De Houwer et al., 2004; Wiers et al., 2002, 2005). Here, it was examined whether support for implicit negative attitudes toward alcohol might be due to contamination of IAT effects by negative extrapersonal information about drinking alcohol that is attitude-irrelevant. To test this hypothesis, we compared a traditional IAT with a personalized IAT (cf. Olson \& Fazio, 2004) that presented individualized stimuli that 
were selected by participants themselves instead of standard stimuli (cf. Fadardi et al., 2006). This was done because standard target (and attribute) concepts do not necessarily evoke the same attitudes in all participants. Results with the standard IAT yielded support for implicit negative attitudes toward alcohol, replicating previous findings. As expected, support for implicit negative attitudes toward alcohol was significantly reduced in the personalized IAT. Contrary to the hypothesis, however, we found no support for implicit positive attitudes toward alcohol with the personalized IAT. Instead, the personalized IAT demonstrated markedly reduced but still significant IAT effects indicative of implicit negative attitudes toward alcohol. Moreover, both the traditional and the personalized IAT were related to alcohol-related behavior and predicted behavior above explicit alcoholrelated expectancies and attitudes.

Consistent with the extrapersonal contamination hypothesis, evidence for implicit negative attitudes toward alcohol was significantly reduced in a personalized IAT that presented individualized stimuli compared to a standard IAT. However, in contrast to the hypothesis, the personalized IAT did not show evidence for implicit positive attitudes toward alcohol. Houben and Wiers (2006b), on the other hand, demonstrated implicit positive attitudes toward alcohol in a personalized IAT that presented standard stimuli without reference to normative feedback. Thus, these results suggest that the personalized IAT used in the present study eliminated extrapersonal contamination to a lesser extent than the personalized IAT used by Houben and Wiers (2006b). Possibly, the individualized attribute stimuli selected by participants in the present study were in fact normatively associated with valence (e.g., love, peace, war, weapons). Hence, a personalized IAT that presents standard stimuli could be more effective in reducing extrapersonal contamination than an individualized personalized IAT because it allows for more controlled selection of stimuli that are not normatively associated with valence. Nonetheless Han, Olson, and Fazio (2006) recently demonstrated that primarily the category labels in the traditional IAT cause vulnerability to extrapersonal contamination and that personalizing the labels in the IAT is sufficient to reduce the impact of extrapersonal associations.

Alternatively, the difference in results with the personalized IAT between the present study and the study by Houben and Wiers (2006b) could be due to differences in the samples of participants. On average, participants in the study by Houben and Wiers (2006b) drank 25 alcoholic consumptions per week whereas in the present study, participants on average consumed less than 16 alcoholic drinks a week. Hence, if implicit positive attitudes towards alcohol are stronger for participants with increased levels of alcohol consumption, as suggested by the findings of Houben and Wiers (2006b), the lower alcohol consumption of participants in the present study compared to participants in the Houben and Wiers (2006b) study can explain why participants in the present study on average demonstrated implicit negative attitudes toward alcohol rather than implicit positive attitudes toward alcohol. 
Importantly, in this study, both the standard and the personalized IAT were related to alcohol-related behavior. Results showed that stronger implicit positive attitudes toward alcohol (or weaker negative attitudes toward alcohol) were related to an increase in alcohol consumption. Moreover, implicit attitudes toward alcohol assessed with both the traditional IAT and the personalized IAT predicted alcohol use above explicit alcoholrelated cognitions. Thus, in line with findings reported by Houben and Wiers (2006b), these results demonstrate incremental predictive validity for the personalized IAT. In addition, incremental predictive validity was also established for the traditional IAT. These findings are consistent with recent studies that also found support for a role of implicit attitudes toward alcohol in alcohol use and abuse with a slight modification of the IAT procedure. When alcohol associations were tested in a unipolar format (e.g., positive vs. neutral and negative vs. neutral) rather than in a bipolar format (e.g., positive vs. negative) so that implicit positive attitudes and implicit negative attitudes could be assessed separately, evidence was found for implicit positive attitudes toward alcohol in addition to strong implicit negative attitudes toward alcohol (Houben \& Wiers, 2006c; Jajodia \& Earleywine, 2003). Moreover, implicit positive attitudes toward alcohol were at least moderately related to alcohol use while implicit negative attitudes toward alcohol did not show a relationship to alcohol-related behavior (Houben \& Wiers, 2006c; Jajodia \& Earleywine, 2003). Together, these findings suggest that strong implicit negative attitudes toward alcohol typically overshadow implicit positive attitudes toward alcohol in an alcohol-IAT that presents positive and negative evaluative categories in a bipolar format.

The present results suggest that implicit negative attitudes toward alcohol at least partly reflect extrapersonal associative knowledge that is unrelated to drinking decisions. In addition, negative evaluative associations with alcohol could also be triggered by the choice of the contrasting target concept soda, which is typically used in alcohol-IATs. Recent studies using a different task than the IAT, namely varieties of the Extrinsic Affective Simon Task (EAST, De Houwer, 2003) that can be used to assess implicit attitudes toward single target concepts, demonstrated positive rather than negative evaluative associations with alcohol that were related to alcohol consumption (De Houwer \& De Bruycker, in press) or indicated implicit ambivalent evaluative associations with alcohol that showed a positive relation to alcohol-related behavior (de Jong, Wiers, van de Braak, \& Huijding, in press). In addition, Houben and Wiers (2006d) recently demonstrated both implicit positive attitudes toward alcohol as well as implicit negative alcohol attitudes with a unipolar Single Target IAT (ST-IAT; Wigboldus, Holland, \& van Knippenberg, 2006) that assessed single implicit attitudes toward alcohol while a unipolar IAT that assessed implicit alcohol attitudes relative to soda only yielded implicit negative attitudes toward alcohol. Importantly, Houben and Wiers (2006d) found that single implicit positive attitudes toward alcohol and implicit positive attitudes toward alcohol relative to soda uniquely predicted alcohol use. 
In sum, consistent across studies that have examined single implicit attitudes toward alcohol using variations of the IAT in order to overcome some of the limitations of the standard IAT procedure as well as variations of the EAST, it has been demonstrated that implicit positive attitudes toward alcohol are an important determinant of alcohol use and abuse (present study; De Houwer \& De Bruycker, in press; de Jong et al., in press; Houben \& Wiers, 2006b, 2006c, 2006d, 2006e; Jajodia \& Earleywine, 2003). Hence, testing implicit alcohol attitudes in a non-comparative format with respect to both the target concept as well as the evaluative categories and using personalized IAT variants in order to eliminate extrapersonal contamination of IAT effects, increases the probability of finding behavior-relevant implicit attitudes toward alcohol. 


\section{APPENDIX}

Target Stimuli in the Traditional IAT

Alcohol: beer, wine, whisky, drink, brandy

Soda: Fanta, Coca Cola, Sprite, sinas (lemonade), ice-tea

Attribute Stimuli in the Traditional IAT

Pleasant: love, sunshine, warmth, peace, hug

Unpleasant: sorrow, war, depression, pain, disease 


$$
\text { D) }
$$




\section{PART II}

\section{BIPOLAR TARGET AND ATTRIBUTE DIMENSIONS IN THE ALCOHOL-IAT: DOUBLE JEOPARDY}




$$
\text { D) }
$$




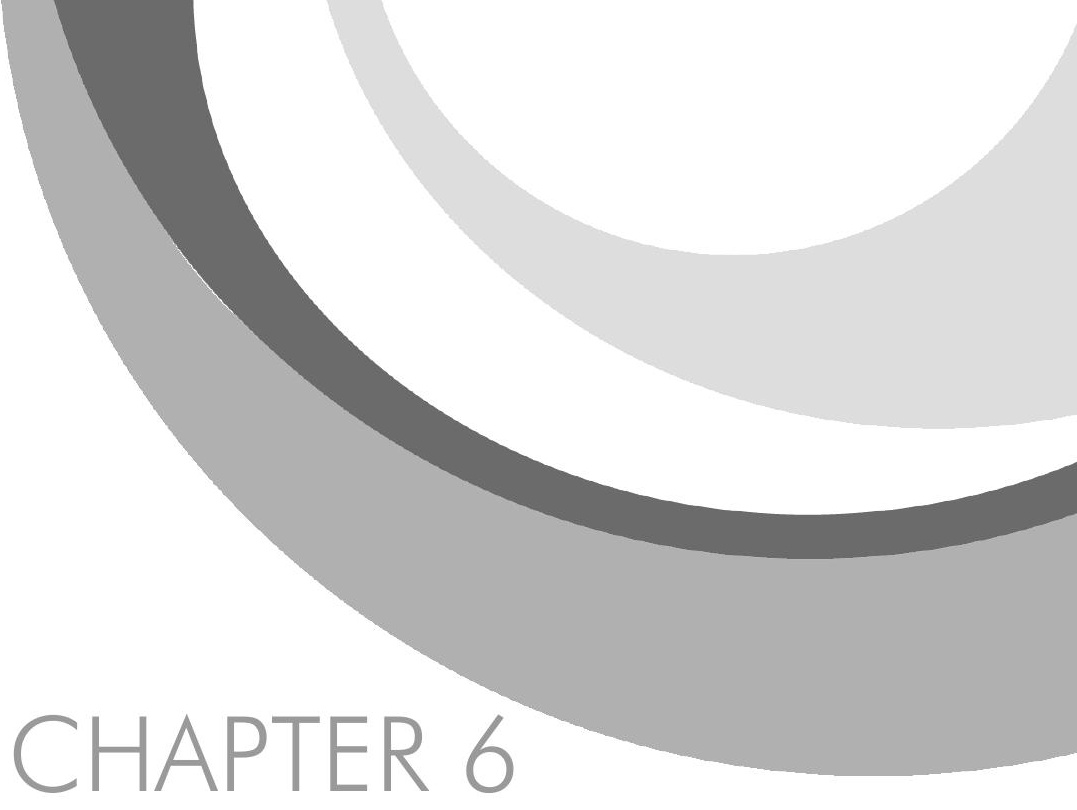

\section{Assessing Implicit Alcohol Associations with the Implicit Association Test: Fact or Artifact?}




\begin{abstract}
Studies using bipolar Implicit Association Tests (IATs) found that heavy drinkers have negative and arousal associations with alcohol relative to soda. Study 1 examined whether these results were due to the label 'alcohol' and the choice of the contrast category 'soda'. Four unipolar IATs assessed alcohol associations with positive and negative valence, arousal, and sedation, while varying the target dimension: alcohol or beer versus soda or animals. Results showed that drinkers had the strongest associations between alcohol and negative valence with the exact strength depending on the choice of the target categories. They also showed associations between alcohol and positive valence, arousal, and to a lesser extent sedation, which were uninfluenced by composition of the target dimension. These findings indicate ambivalence in both the valence and arousal-sedation dimension, underscoring the importance of using unipolar alcohol-IATs. Further, study 2 showed that "Figure-Ground" asymmetries could not account for these IAT results. These findings provide support that implicit alcohol associations are not merely IAT artifacts and that they can be assessed in a meaningful way with unipolar IATs.
\end{abstract}

\title{
INTRODUCTION
}

The past decade, alcohol expectancies have been shown to be powerful predictors of drinking and it is now believed that they act as a common pathway for the influence of more distal risk factors for alcohol abuse (e.g., Goldman, 1999). Further, multidimensional scaling (MDS) research demonstrated that there are two basic dimensions underlying alcohol expectancies: a positive-negative and an arousal-sedation dimension. While expectancies in the arousal-sedation dimension have been found to discriminate between drinking levels, no relation has been found between expectancies in the valence dimension and drinking (e.g., Rather \& Goldman, 1994). This explanatory power of expectancy theory inspired researchers to develop a variety of expectancy measurement instruments that almost exclusively rely on self-report. Self-report measures, however, have been criticized because of their susceptibility to self-presentation biases and the possibility that cognitive processes mediating alcohol abuse are not accessible through introspection (McCusker, 2001; Nisbett \& Wilson, 1977; Stacy, 1997). Therefore, alcohol research is now exploring the value of implicit measures, such as the now widely used Implicit Association Task (IAT; Greenwald, McGhee, \& Schwartz, 1998), that assess cognitions indirectly, without asking people to reflect and report on the motivations for their behavior. 
The IAT is a double categorization task during which stimuli are classified into two times two categories with two response keys. Typically, two categories represent the target concepts (e.g., alcohol versus soda) and two categories correspond to the poles of an attribute dimension (e.g., positive versus negative). During the critical IAT trials, the target and attribute categories are assigned to two response keys in two different combinations. The assumption is that performance will be superior when associated concepts are assigned to the same response key (compatible combination), than when concepts sharing a response key are not or only weakly associated (incompatible combination). The performance difference between the two combination tasks, or the IAT effect, reflects the strength of implicit associations between the target and the attribute categories (Greenwald et al., 1998).

\section{IMPLICIT ALCOHOL ASSOCIATIONS}

Wiers, van Woerden, Smulders, and de Jong (2002) used the IAT to examine heavy and light drinkers' implicit alcohol associations with outcomes in both the arousal and valence expectancy dimension. Results showed that heavy drinkers, but not light drinkers, had implicit alcohol-arousal associations, paralleling results from MDS expectancy research. Surprisingly, and in contrast to MDS research, both light and heavy drinkers strongly associated alcohol with negative outcomes. These findings with the alcohol-IAT have been replicated in a sample of heavy drinkers (Wiers, van de Luitgaarden, van den Wildenberg, \& Smulders, 2005) and in a sample of patient drinkers (De Houwer, Crombez, Koster, \& De Beul, 2004).

Wiers et al. (2002) linked the finding of implicit alcohol-arousal associations to the incentive-sensitization theory of addiction which states that addictive substances sensitize brain systems mediating processes responsible for motivating behavior and determining incentive salience ("wanting"), but not brain systems mediating the hedonic effects of addictive substances ("liking"). Once sensitized, this "wanting" system can be implicitly activated and automatically increases attention, arousal, and goal-directed behavior (Robinson \& Berridge, 1993; 2003). With respect to the finding of negative alcohol associations, three plausible explanations are available. First, Jones and McMahon (1996) argue that negative alcohol expectancies increase with consumption but without influencing drinking behavior until a certain threshold is reached. Second, implicit negative associations possibly reflect past, negative experiences whereas explicit cognitions may stem from more recent, positive experiences. Preliminary support for this account has already been obtained in relation to smoking (Rudman, 2004; Rudman and Heppen, 2003). Third, the negative-alcohol IAT effect could be due to an artifact of the IAT procedure. 


\section{METHODOLOGICAL ISSUES SURROUNDING THE IAT}

With respect to the IAT target dimension, there are two main problems. First, De Houwer (2001) argues that the IAT primarily measures associations at category level and that category labels determine IAT effects more strongly than exemplars (i.e., the 'label effect'). Additionally, IAT effects are prone to contamination by culturally shared associative knowledge (Karpinski \& Hilton, 2001; Olson \& Fazio, 2004). Hence, IAT results can be caused by cultural connotations carried by the category labels and superior IAT performance when alcohol and negative are mapped together could thus be due to negative cultural connotations of the label 'alcohol'11. Second, the IAT is a relative measure: it compares the strength of implicit associations of one target category to those of the contrast category. As a result, faster performance when alcohol and negative (and soda and positive) are paired may be due to negative alcohol associations, positive soda associations or a combination of both.

Another problem with the IAT is that the attribute dimension is bipolar and that the test is therefore unfit for assessing ambivalent associations. This could be especially problematic when assessing implicit alcohol associations, since attitudes towards alcohol have been found to be highly ambivalent (Conner \& Sparks, 2002). Therefore, Jajodia and Earleywine (2003) modified the IAT by contrasting positive and negative categories with neutral ones. When attribute categories were tested in this unipolar format, performance was facilitated for both the combination of alcohol and positive words and the pairing of alcohol and negative words relative to the combination of mammals and neutral words. However, since the positive expectancy IAT was always performed before the negative expectancy IAT, order effects could have influenced results. It is a common finding that IAT effects are considerably larger the first time the task is performed and decrease with experience (Geenwald, Nosek, \& Banaji, 2003; Wiers et al., 2005).

Finally, IAT effects could reflect non-associative factors rather than implicit associations. Rothermund and Wentura (2004) proposed such a non-associative account of IAT effects that is based on salience. In a series of studies, they showed that salience or figure-ground asymmetries can produce IAT effects independent of implicit associations. When categories of an IAT dimension differ in salience, the salient category will act as figure against the less salient (back)ground category. Consequently, performing the IAT should be easier for consistent mappings of the figure categories of both IAT dimensions than when one figure and one ground category are assigned to the same response.

Two studies were designed that explored whether previous results with the alcohol-IAT could have been caused by these limitations. Study 1 examined the effect of using a contrast

\footnotetext{
${ }^{11}$ Public health campaigns in The Netherlands (and many other countries) typically stress the negative consequences of drinking alcohol whereas advertisements generally emphasize the positive and pleasant effects of drinking different brands of beer.
} 
category other than soda on results with the alcohol-IAT as well as the effect of the label 'alcohol'. Also, the value of assessing implicit alcohol associations in a unipolar format was further explored. Study 2 examined whether the IAT results of study 1 could reflect salience asymmetries, instead of, or in addition to, implicit alcohol associations. To rule out such an alternative explanation, salience asymmetries between categories that formed an IAT dimension in study 1 were examined with visual search tasks which, according to Rothermund and Wentura (2004), can be used to objectively assess salience asymmetries.

\section{STUDY 1}

Four IAT versions were compared that differed with respect to the target dimension: alcohol vs. soda (cf. Wiers et al., 2002), alcohol vs. animals (cf. Jajodia \& Earleywine, 2003) ${ }^{12}$, beer vs. soda, or beer vs. animals. Further, the attribute categories positive, negative, arousal and sedation were tested against neutral attribute categories to examine the utility of unipolar alcohol-IATs. The IAT was expected to show both a strong negative attitude and a weak positive attitude towards alcohol whereas the opposite was expected for beer ${ }^{11}$. Also, it was hypothesized that alcohol would be associated with arousal and that these associations would be related to alcohol use and problems, reflecting the activation of a sensitized "wanting" system. In addition, the relationship between these IAT effects and alcohol-related attitudes and expectancies was examined as well as the relation to recent and early alcoholrelated experiences and social messages about alcohol use. IAT effects were expected to be related to both early alcohol experiences and social messages while self-reported attitudes and expectancies were expected to correlate with recent alcohol experiences.

\section{METHOD}

\section{Participants}

Ninety-six students of Maastricht University (48 males; mean age $=20.4$ years, $S D=2.51$ ) participated in return for course credit or a gift certificate of $10 €$. Participants at least occasionally drank alcohol, including beer with an average weekly alcohol consumption of 15.07 (SD = 10.55) Dutch standard drinks ${ }^{13}$. Participants had an mean score of 9.90 (SD = 3.73) on the Alcohol Use Disorder Identification Test (AUDIT; Saunders, Aasland, Babor, De

\footnotetext{
${ }^{12}$ Unlike in the study of Jajodia and Earleywine (2003), the animals category used here did not exclusively consist of positively evaluated mammals but instead of atypical animals, which were evaluated as neutral.

${ }^{13}$ A standard alcoholic drink in Holland contains less alcohol than a standard English or American alcoholic drink: 10 vs. $14 \mathrm{~g}$, respectively.
} 
la Fuente, \& Grant, 1993), which is comparable to American college students (Fleming, Barry, \& MacDonald, 1991), and an average item score of .47 (SD = .33) on the 18-items version of the Rutgers Alcohol Problem Index (RAPI; White \& Labouvie, 2000). The average item score in clinical samples is about .80 (White \& Labouvie, 1989).

\section{Materials and Measures}

Alcohol Use. Alcohol use was assessed with a self-report questionnaire based on the timeline follow-back method (Sobell \& Sobell, 1990). Participants were asked to indicate how many drinks of different types of alcoholic drinks they consumed on each day of the past week, and for each day of the week, how many drinks they typically consumed on this day.

Alcohol-Related Problems. Alcohol-related problems were assessed with the RAPI and the AUDIT. The RAPI described 18 alcohol-related problem situations and participants indicated how often they experienced these situations on a 5 -point Likert scale $(0=$ never, 4 = often) (Cronbach $\alpha=.67)$. The AUDIT consisted of 10 multiple choice questions. The first three questions related to alcohol use, the other seven to alcohol-related problems $(\alpha$ $=.76)$.

Implicit Alcohol Associations. Participants performed either an alcohol-soda IAT, an alcohol-animals IAT, a beer-soda IAT or a beer-animals IAT. All (Dutch) target categories were matched on familiarity, valence, arousal and number of syllables. Each IAT version assessed associations in four evaluative dimensions: positive vs. neutral, negative vs. neutral, arousal vs. neutral, and sedation vs. neutral. The positive (label 'pleasant') and negative (label 'unpleasant') categories consisted of positive and negative nouns (cf. Greenwald et al., 1998) to assess general attitudes towards the target concepts. The arousal (label 'active ${ }^{\prime 14}$ ) and sedation (label 'quiet ${ }^{\prime 14}$ ) categories consisted of outcome expectancies (cf. Wiers et al., 2002) to assess associations with drinking outcomes. The (Dutch) positive, negative and their paired (in balanced order) neutral categories were matched on familiarity, arousal (neutral on arousal) and number of syllables. Likewise, the (Dutch) arousal, sedation and their paired (in balanced order) neutral categories were matched on familiarity, valence (neutral on valence) and number of syllables. All stimuli are presented in the Appendix. Internal consistencies, calculated as in Greenwald et al. (2003), were .46 for the positive dimension, .44 for the negative dimension, .52 for the arousal dimension, and .46 for the sedation dimension.

All IAT versions were programmed in ERTS 3.18 (Beringer, 1996) and consisted of seventeen blocks. Participants first received 24 trials of target discrimination practice using

\footnotetext{
${ }^{14}$ The labels 'active' and 'quiet' were chosen for the arousal and sedation category, respectively, because there are no suitable terms to denote 'arousal' and 'sedation' in Dutch.
} 
a right and a left response key. All target stimuli were presented twice. In the second block, the attribute classification (e.g., pleasant vs. neutral) was practiced with the same response keys. All attribute stimuli were presented twice. The third block was a combination block during which both target and attribute stimuli were presented twice for a total of 48 trials. Next, participants practiced the reversed attribute discrimination, followed by the reversed combination block. Blocks 2 to 5 were then repeated for the other three attribute dimensions. Stimuli were presented randomly with the restriction that targets and attributes were presented in alternating order (Greenwald et al., 1998). Stimuli appeared in the middle of the computer screen, in black against a grey background. Instructions were presented before each task. Category labels were presented in the upper corners of the computer screen in agreement with the required response and remained there during the task. Stimuli remained on screen until a response was given. Feedback ('wrong', 'too fast' $[<300 \mathrm{~ms}$ ] and 'too slow' [>3000 ms]) was presented in red beneath the stimuli. The intertrialinterval was $250 \mathrm{~ms}$.

Thought-Listing. The thought-listing task (Rudman \& Heppen, 2003) asked participants to report five thoughts that came to mind quickly and easily about recent and early alcohol-related experiences and social messages about alcohol. Participants then indicated for each thought whether it was positive or negative on a 6-point Likert scale (-3 = extremely negative, +3 = extremely positive) They also rated the personal importance of all recent and early alcohol experiences on a 5-point Likert scale $(1=$ not at all important, 5 = extremely important) and they estimated how often they encountered the social messages they listed on a 5 -point Likert scale ( $1=$ seldom, $5=$ all the time).

Explicit Alcohol-Related Cognitions. Explicit alcohol-related cognitions were assessed with an expectancy questionnaire, an attitude questionnaire and a feeling thermometer. The alcohol expectancy questionnaire consisted of 6 positive $(\alpha=.85), 6$ negative $(\alpha=.91), 7$ arousal $(\alpha=.65)$ and 7 sedation expectancy items $(\alpha=.65)$. Each item asked participants to indicate on a Visual Analogue Scale (VAS) how much they agreed or disagreed with the statement: "After drinking alcohol, I feel . ..". For the positive and negative items, this statement was completed with the words unhappy, depressive, sad, lonely, moody, down, pleasant, happy, sociable, friendly, enjoyable, and likable. For the arousal and sedation statements, the same words as presented during the IAT were used, including the labels. The alcohol attitude questionnaire consisted of 4 semantic differentials which asked participants to indicate on a VAS how much they considered drinking alcohol to be unpleasant-pleasant, bad-good, boring-fun, and stupid-smart. The first and third item formed an affective attitude component $(\alpha=.90)$, the other two items formed a cognitive attitude component ( $\alpha=.77$ ). Finally, two feeling thermometers, labeled in 10 degree increments ranging from 0 (cold) to 100 (warm), asked participants how favorable they felt about the target concepts (depending on condition: alcohol/beer and soda/animals). 


\section{Procedure}

Participants first filled out an informed consent form. Next, they performed one of the four IAT versions. The response assignment of the target categories was balanced across participants. The presentation order of the attribute dimensions was partially balanced with a Latin square, resulting in 4 orders. Participants first performed the IAT with alcohol/beer paired with the relevant attribute category (positive, negative, arousal and sedation) and then with alcohol/beer paired with the neutral category. Participants then received the thought-listing questionnaire, the feeling thermometer, the alcohol attitude questionnaire and the alcohol expectancy questionnaire. These questionnaires referred to either alcohol or beer, depending on the IAT condition. Finally, participants received the alcohol use questionnaire, the AUDIT and the RAPI, and rated all IAT stimuli on familiarity, valence and arousal.

\section{RESULTS}

\section{Implicit Alcohol Associations}

First, it was examined whether there were differences in reported alcohol use and problems between IAT conditions using 2 (target: alcohol or beer) $\times 2$ (contrast: soda or animals) univariate analyses of variance (ANOVA). Results showed no effect of target $(p>.25)$ or contrast $(p>90)$ on reported alcohol use and a borderline significant effect of target $(p=.06)$ but no effect of contrast $(p>.90)$ on reported alcohol-related problems ${ }^{15}$. Next, IAT effects were calculated with the new D600 algorithm (Greenwald et al., 2003) in such a way that higher IAT scores reflect a stronger association between alcohol/beer and positive, negative, arousal, or sedation. Then, the effect of target and contrast category on IAT effects for each evaluative dimension (positive, negative, arousal and sedation) was examined with a 2 (target: alcohol or beer) $\times 2$ (contrast: soda or animals) ANOVA $^{16}$. No significant effect of target was found for the positive, arousal and sedation dimension $(p>.90)$. For the positive and sedation dimension, the effect of contrast category was also not significant $(p>.15)$, while borderline significant for the arousal dimension, $F(1,91)=3.11, p=.081$. For the negative dimension, there was a significant effect of both target, $F(1,91)=4.28, p=.041$, and contrast category, $F(1,91)=14.14, p<.001$. The interaction between target and contrast category was borderline significant, $F(1,91)=3.76, p=.056$. In sum, the target label and the choice of the contrast category only influenced results for the negative IAT dimension.

\footnotetext{
15 There was a borderline significant trend that showed higher reporting of alcohol-related problems in the alcohol condition compared with the beer condition. Therefore, the factor target (alcohol vs. beer) was entered in the hierarchical regression analysis of alcohol problems in step 1. Results showed that the pattern of results remained the same.

${ }_{16}$ Preparatory analyses revealed the presence of an influential outlier who scored more than 4 standard deviations above the mean for the conventional measure on the arousal IAT dimension. This participant was excluded from the analyses. Outcomes were checked for the original sample including the outlier and results were generally the same.
} 
For the positive, arousal and sedation dimension, the IAT effects were as follows (averaged across target and contrast categories): performance was faster when alcohol and positive words were assigned to the same response, $f(94)=7.67, p<.001(d=.78)$, when alcohol and arousal were mapped onto the same response, $t(94)=7.15, p<.001(d=.73)$, and when alcohol and sedation were paired, $t(94)=4.61, p<.001(d=.47)$ compared to when alcohol and neutral words shared a response. These findings demonstrate that drinkers hold strong implicit alcohol associations with positive valence, and with arousal and also to a lesser extent with sedation. For the negative evaluative dimension, planned comparisons between the four IAT versions showed that the IAT effect was significantly smaller in the beeranimals IAT than in both the beer-soda IAT, $t(46)=4.25, p<.001$ and the alcohol-animals IAT, $t(45)=3.01, p=.004$. No significant differences emerged between the alcohol-soda and the alcohol-animals IAT, $t(45)=1.23, p=.227$, nor between the alcohol-soda IAT and the beer-soda IAT, $t(46)=.087, p>.50$. These results show that the IAT effect was smaller in the beer-animals IAT than in the other IAT versions. Nevertheless, IAT performance was faster when alcohol and negative words shared a response than when alcohol and neutral words shared response in all four IAT versions: $t(23)=10.03, p<.001(d=2.05)$ for the alcohol-soda IAT, $t(22)=9.48, p<.001(d=1.98)$ for the alcohol-animals IAT, $t(23)=11.06, p<.001(d=$ 2.26) for the beer-soda IAT and $t(23)=5.89, p<.001(d=1.2)$ for the beer-animals IAT. These results indicate that drinkers strongly associate alcohol with negative valence and that the size of this implicit association is sensitive to the wording of both the target and contrast category. IAT effects for all evaluative dimensions per IAT version are shown in Figure 1. Paired samples t-tests showed that IAT effects for the negative and the arousal dimension were significantly larger than IAT effects for the positive, $t(94)=5.41, p<.001$, and the sedation dimension, $t(94)=2.60, p=.011$, respectively.

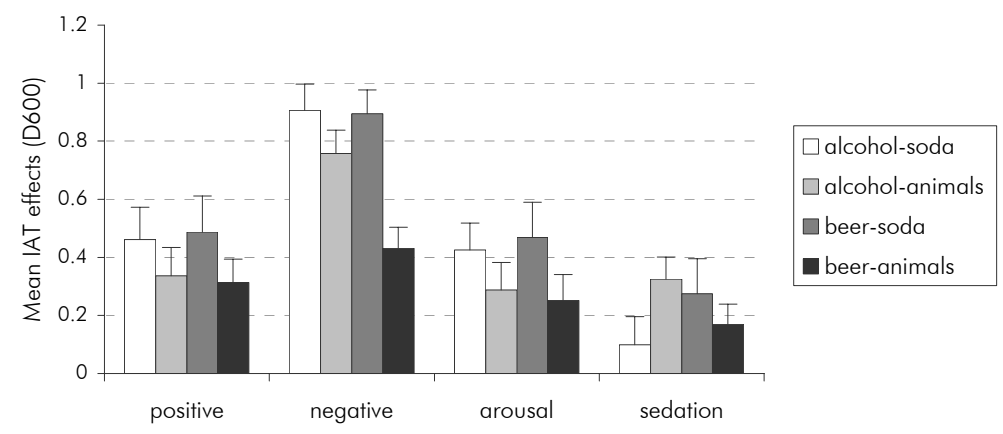

Figure 1. Mean IAT effects (D600) separately for each evaluative IAT dimension and IAT version. 


\section{Explicit Alcohol-Related Cognitions}

Participants' mean score for positive and arousal alcohol expectancies significantly deviated from the neutral midpoint of the scale (55), $M=73.29, S D=13.88, t(94)=$ $12.84, p<.001(d=1.32)$, and $M=59.59, S D=12.29, t(94)=3.63, p<.001$ ( $d$ $=.37$ ) respectively, indicating that they agreed with positive and arousal expectancies. Participants also significantly disagreed with negative and with sedation expectancy statements, $M=18.75, S D=15.35, t(94)=-23.02, p<.001(d=2.36)$, and $M=$ 41.32, $S D=13.62, t(94)=-9.79, p<.001(d=1.01)$, respectively. Analysis of mean scores on the VAS for both the affective and cognitive alcohol attitude component showed that participants had a positive attitude towards alcohol, $M=80.57, S D=14.02, t(94)$ $=14.65, p<.001(d=1.50)$, while knowing that drinking alcohol is unwise, $M=$ 46.33, $S D=17.07,+(94)=-4.95, p<.001(d=.51)$. With respect to the feeling thermometers, paired samples t-tests showed that feelings towards animals $(M=77.92$, $S D=15.05)$ were more favorable than feelings towards both alcohol $(M=66.88, S D=$ $14.79), t(23)=-2.14, p=.044$, and beer $(M=65.21, S D=16.38), t(23)=-3.31, p$ $=.003$. Feelings towards soda $(M=65.32, S D=14.23)$, in contrast, did not differ from feelings toward alcohol, $t(23)=-.19, p>.50$, or beer, $t(22)=.42, p>.50$. Before performing correlational analyses, all explicit measures and implicit D600 IAT scores were z-transformed per IAT condition (i.e. beer or alcohol vs. soda or animals). As can be seen in Table 1, IAT effects for both the positive and arousal dimension were significantly correlated with the affective attitude component and with feelings towards the target category, while a significant negative correlation emerged with feelings towards the contrast category. Also, IAT effects for both the positive and sedation dimension correlated positively with the cognitive attitude component. None of other correlations reached significance ${ }^{17}$.

\section{Alcohol Use and Related Problems}

Estimates of alcohol consumption during the past week and of average weekly alcohol consumption were calculated from the alcohol use questionnaire. The weighed mean of these two z-transformed estimates and the z-transformed sum score of the first three AUDIT items was calculated as an estimate of mean alcohol use. Correlations between this alcohol use estimate and implicit and explicit measures are presented in Table 1. The alcohol use estimate correlated positively with the arousal IAT, with positive and arousal expectancies, with both the affective and cognitive attitude component and with feelings toward the target category. Alcohol use was also negatively correlated with negative expectancies.

\footnotetext{
${ }^{17}$ When the non-standardized explicit and implicit measures were used in the analysis, the pattern of results was generally the same.
} 


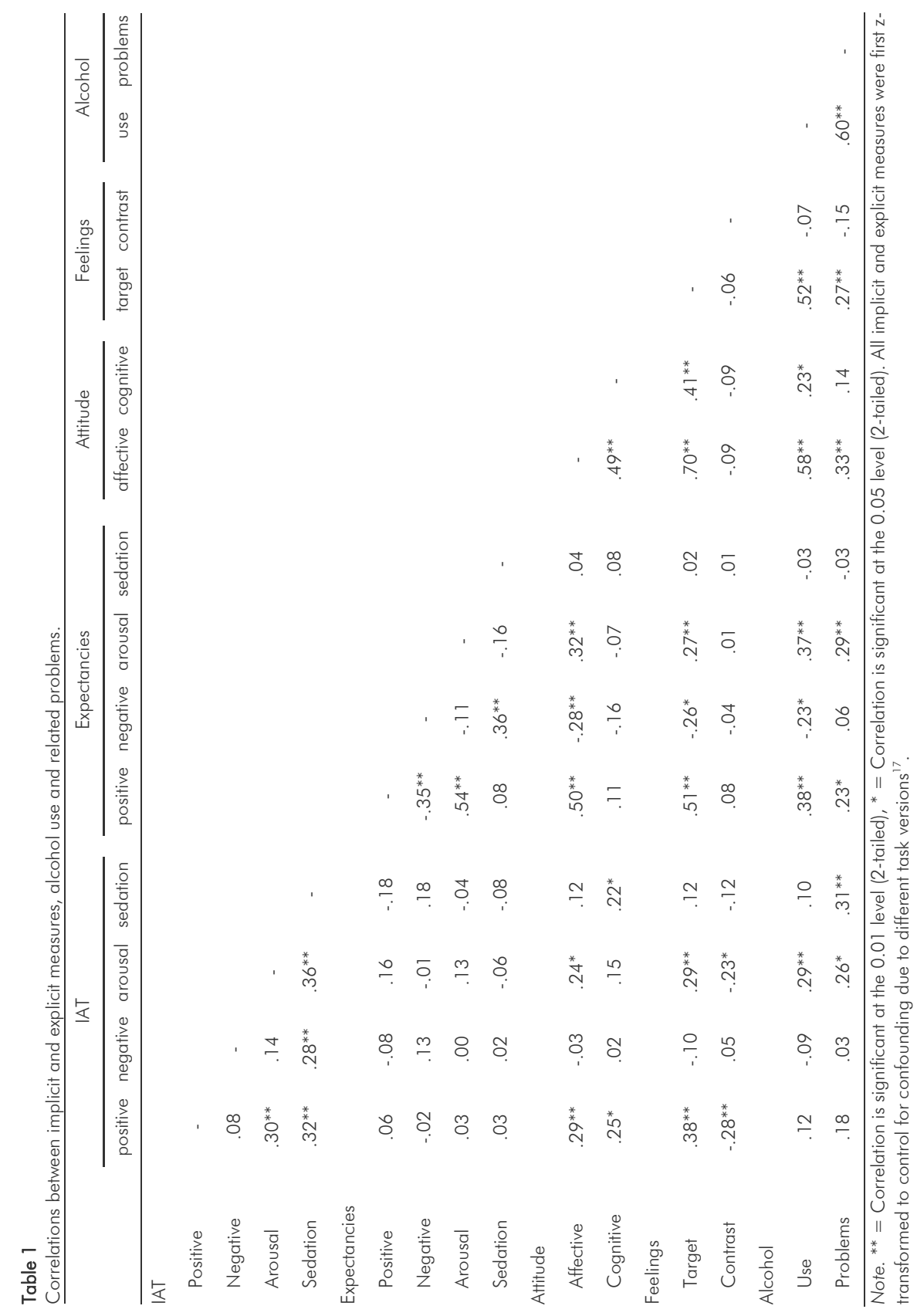


The alcohol use estimate was then entered into a hierarchical multiple regression analysis. All z-transformed explicit measures and implicit associations were entered in the first run of the regression analysis. All variables that did not predict $(p>30)$ alcohol use were left out in the second run of the regression analysis. Results of the second run of the regression analysis for the prediction of alcohol use are shown in Table $2^{17}$. In step 1, only gender was entered which significantly predicted alcohol use (men drink more than women). In step 2, the explicit arousal expectancies, affective attitude component and target feeling thermometer were entered which significantly increased the variance explained. Both arousal expectancies and the affective attitude component significantly predicted alcohol use. In step 3, entering implicit positive and arousal associations significantly increased the variance explained. Implicit arousal associations significantly predicted alcohol use while implicit positive associations were borderline significant predictors.

Table 2

Summary of hierarchical regression analysis for explicit and implicit measures predicting alcohol use.

\begin{tabular}{|c|c|c|c|c|c|c|c|}
\hline Step & Variable & B & SE B & $\beta$ & SE $\beta$ & $t$ & $p$ \\
\hline 1 & Gender & -.40 & .17 & -.24 & .10 & -2.43 & .017 \\
\hline \multirow[t]{4}{*}{2} & Gender & -.27 & .14 & -.16 & .08 & -1.94 & .056 \\
\hline & Arousal expectancies & .18 & .07 & .22 & .09 & 2.52 & .013 \\
\hline & Affective attitude & .28 & .10 & .33 & .12 & 2.81 & .006 \\
\hline & Target feeling thermometer & .17 & .10 & .20 & .11 & 1.79 & .076 \\
\hline \multirow[t]{6}{*}{3} & Gender & -.26 & .14 & -.16 & .09 & -1.84 & .069 \\
\hline & Arousal expectancies & .16 & .07 & .20 & .09 & 2.32 & .023 \\
\hline & Affective attitude & .27 & .10 & .33 & .11 & 2.87 & .005 \\
\hline & Target feeling thermometer & .19 & .10 & .22 & .12 & 1.91 & .060 \\
\hline & Implicit positive associations & -.14 & .08 & -.16 & .09 & -1.81 & .073 \\
\hline & Implicit arousal associations & .15 & .07 & .18 & .09 & 2.07 & .041 \\
\hline
\end{tabular}

An estimate of alcohol-related problems was computed as the weighed mean of the ztransformed RAPI sum score and the z-transformed sum score of the last seven AUDIT items. Because this estimate was not normally distributed, the RAPI and AUDIT sum scores were log-transformed before z-transformation. Correlations between the alcohol-related problems estimate and implicit and explicit measures are presented in Table 1. Alcoholrelated problems correlated positively with arousal and sedation IAT scores, positive and arousal expectancies, the affective attitude component, and feelings toward the target 
category. The alcohol-related problems estimate was entered into a hierarchical multiple regression analysis, using the same procedure as described above. Results of the second run of the regression analysis for the prediction of alcohol-related problems are shown in Table $3^{17}$. In step 1, gender was entered which did not predict alcohol-related problems. In step 2, arousal and negative expectancies, and the affective attitude component were entered, which significantly increased the variance explained. Both arousal expectancies and the affective attitude component predicted alcohol-related problems. Entering implicit sedation associations in step 3 significantly increased the variance explained.

Table 3

Summary of hierarchical regression analysis for explicit and implicit measures predicting alcohol-related problems.

\begin{tabular}{|c|c|c|c|c|c|c|c|}
\hline Step & Variable & $B$ & SE B & $\beta$ & SE $\beta$ & $t$ & p \\
\hline 1 & Gender & -.27 & .19 & -.15 & .10 & -1.48 & .142 \\
\hline \multirow[t]{4}{*}{2} & Gender & -.18 & .18 & -.10 & .10 & -1.01 & .315 \\
\hline & Arousal expectancies & .20 & .09 & .22 & .10 & 2.19 & .031 \\
\hline & Negative expectancies & .14 & .09 & .16 & .10 & 1.57 & .120 \\
\hline & Affective attitude & .26 & .10 & .29 & .11 & 2.64 & .010 \\
\hline \multirow[t]{5}{*}{3} & Gender & -.17 & .17 & -.09 & .10 & -.95 & .345 \\
\hline & Arousal expectancies & .22 & .09 & .24 & .10 & 2.44 & .016 \\
\hline & Negative expectancies & .09 & .09 & .10 & .10 & 1.01 & .315 \\
\hline & Affective attitude & .21 & .10 & .23 & .11 & 2.20 & .030 \\
\hline & Implicit sedation associations & .24 & .09 & .26 & .10 & 2.73 & .008 \\
\hline
\end{tabular}

Note. $F(1,93)=2.20, p=.142, R^{2}=.02$ for step $1 ; F_{\text {change }}(3,90)=5.91, p=.001, R_{\text {change }}=.16 ; F_{\text {change }}(1,89)$ $=7.42, p=.008, R_{\text {change }}^{2}=.06$ for step 3. Final model $R^{2}=.25, R_{\text {odiusted }}^{2}=.20, F(5,94)=5.83, p<.001$.

\section{Thought-Listing}

Indices were formed by multiplying valence scores of every recent and early thought with its assigned personal significance. For social messages, indexes were calculated by multiplying each message's valence with its frequency. Mean indices were then computed for recent experiences, earliest experiences and social messages. Recent experiences were more positive than both early experiences, $t(95)=5.50, p<.001$, and social messages, $t(95)=5.99$. No difference was found between the valence of early experiences and social messages, $t(95)=1.08, p=.283$. Correlations of $z$-transformed thought indices and implicit and explicit measures are presented in Table $4^{17}$. Recent and early alcohol experiences correlated positively with positive IAT scores, the two attitude components and feelings towards alcohol. Also, recent alcohol experiences correlated positively with positive expectancies and negatively with negative expectancies, whereas early alcohol 
experiences correlated positively with both arousal IAT scores and alcohol use. Unexpectedly, social messages correlated positively with both the cognitive attitude component and negative IAT scores.

Table 4

Correlations between reported thoughts and implicit and explicit measures.

\begin{tabular}{|c|c|c|c|}
\hline & Recent experiences & Early experiences & Social messages \\
\hline Positive IAT & $.27^{* *}$ & $.33^{* *}$ & .02 \\
\hline Negative IAT & .00 & -.15 & $.22^{*}$ \\
\hline Arousal IAT & .14 & $.23^{*}$ & -.04 \\
\hline Sedation IAT & .09 & .20 & .13 \\
\hline Positive expectancies & $.34^{* *}$ & .18 & -.07 \\
\hline Negative expectancies & $-.23^{*}$ & -.08 & -.10 \\
\hline Arousal expectancies & .11 & .13 & -.05 \\
\hline Sedation expectancies & .01 & .02 & .01 \\
\hline Affective attitude & $.33^{* *}$ & $.39^{* *}$ & .07 \\
\hline Cognitive attitude & $.22^{*}$ & $.24^{*}$ & $.24^{*}$ \\
\hline Feelings target & $.36^{* *}$ & $.32^{* *}$ & -.03 \\
\hline Feelings contrast & -.09 & -.19 & .14 \\
\hline Alcohol use & .16 & $.23^{*}$ & -.08 \\
\hline Alcohol problems & .08 & .19 & -.07 \\
\hline
\end{tabular}

\section{DISCUSSION}

The present results show that drinkers hold strong implicit associations between alcohol and negative valence $(d>1)$, with the exact size of this effect depending on the choice of the target categories (alcohol or beer vs. animals or sodas). Additionally, drinkers held significant (but weaker) associations between alcohol and positive valence, arousal, and to a lesser extent sedation, and these associations did not depend on the choice of the target categories. These findings indicate ambivalence in both the valence and the arousal-sedation dimension, underscoring the need to assess implicit alcohol associations in a unipolar format. Also, these results provide support that implicit alcohol associations are not merely IAT artifacts related to choice of the contrast target category and the target category labels. Nevertheless, the strong negative alcohol associations found in the IAT still contrast sharply with the scores on self-report measures. An alternative explanation for the present results is 
that the IAT reflects non-associative factors, instead of alcohol associations. This explanation is not implausible given that negative words typically acts as figure (Rothermund \& Wentura, 2004) and that alcohol may be more salient in heavy drinkers (attentional bias).

\section{STUDY 2}

Study 2 assessed salience asymmetries between categories that formed an IAT dimension in study 1 with the visual search task. This task simultaneously presents four stimuli and participants have to decide if all stimuli belong to the same category or not. Since it takes longer to process salient stimuli than non-salient stimuli, responses will be slower when the majority of stimuli (i.e., distractors) belong to the figure category than when they belong to the ground category (Rothermund \& Wentura, 2004). Hence, salience asymmetries are assessed by comparing performance when distractors belong to one category of an IAT dimension with performance when distractors belong to the other category of that dimension.

\section{METHOD}

\section{Participants}

A sample of 39 participants of study 1 ( 15 male; mean age $=20.5$ years, $S D=2.31$ ), participated in return for course credit or a gift certificate of $7.5 €$.

\section{Materials and Measures}

Visual Search Tasks. Participants performed eight visual search tasks, one for each IAT dimension presented in study 1. Four visual search tasks assessed salience asymmetries between the target categories, the other four measured salience asymmetries between attribute categories. Visual search tasks were programmed in ERTS 3.18 (Beringer, 1996), following Rothermund and Wentura (2004). Before each visual search task, participants received 20 trials of a simple categorization task to practice the categorization of the stimuli. During these practice trials, each stimulus was presented twice. Participants were instructed to classify these stimuli with two response keys as quickly and as accurate as possible. After a wrong response, feedback was displayed beneath the stimulus ('ERROR - press correct key and continue') and the stimulus remained on the screen until the correct response was given. The intertrialinterval was $250 \mathrm{~ms}$.

Next, the corresponding visual search task was performed. Each visual search task consisted of 12 practice and 64 test trials. During each trial, four stimuli were presented simultaneously. On half the trials, all four stimuli belonged to the same category ('same' trials), on the other half, three stimuli belonged to one category and the fourth (i.e., 
target) stimulus belonged to the other category ('different' trials). Participants indicated with two response keys whether all stimuli belonged to the same category or not. On half of the trials, distractors (three or four out of four stimuli) belonged to the first category of the respective dimension, on the other half of the trials, the distractors belonged to the second category. Stimuli were presented in the form of a square on half of the trials, and in the form of a diamond on the other half of the trials. During the different trials, the target appeared twice at each of the four possible locations. Each trial started with a ready signal $\left({ }^{\prime} X '\right)$ displayed in the middle of the screen which was replaced by a cue $\left(^{(* \prime}\right)$ when participants pressed the space bar. After 500 ms, four stimuli were presented around the cue. Stimuli were always presented in black against a grey background. Instructions were presented before each visual search task. Response labels (same or different) were presented in the upper corners of the computer screen, in accordance with the response assignment and remained there for the duration of the task. Stimuli remained on screen until the correct response was given. In case of an error, feedback ('ERROR - press correct key and continue') was presented in red beneath the stimuli until the correct response was given.

\section{Procedure}

After filling out an informed consent form, participants performed all visual search tasks. The order of visual search tasks was partially balanced with a Latin square, resulting in 8 orders. The response assignment of same and different trials was balanced.

\section{RESULTS \& DISCUSSION}

Similar to Rothermund and Wentura (2004), trials with erroneous responses were left out of the analyses. For each visual search task, same and different trials were analyzed together, by computing mean reaction times for trials with distractors of the first category of the dimension (e.g., alcohol) and for trials with distractors of the other category (e.g., soda). No significant differences in responding were found between the target categories: $t(38)=1.67, p=.103$ for the alcohol-soda dimension, $t(38)=-.92, p=.363$ for the alcohol-animals dimension, $t(38)=-1.61, p=.115$ for the beer-soda dimension, and $t(38)=-1.28, p=.210$ for the beer-animals dimension. Thus, there was no evidence for salience asymmetries within the target dimensions. For the attribute dimensions, results indicated that responding to neutral distractors was significantly slower than responding to positive, $t(38)=-3.86, p<.001$, negative, $t(38)=-2.21, p=.033$, and sedation distractors, $t(38)=-2.87, p=.007$. No such effect was found for the arousal-neutral dimension, $t(38)=-.76, p=.452$. Hence, the neutral attribute categories appeared to be more salient than the positive, negative and sedation category. It should be noted, however, that the neutral stimuli possibly constituted a 
more heterogeneous sets than the positive, negative, arousal and sedation categories which could also have impaired performance during neutral trials. Nevertheless, no salience asymmetry was found for the arousal dimension. The present findings, consequently, imply that salience asymmetries cannot account for the IAT effects found in study 1 .

\section{GENERAL DISCUSSION}

The present research aimed at replicating and extending previous results with bipolar alcohol-IATs that showed that alcohol, relative to soda, is associated with both negative valence and arousal (De Houwer et al., 2004; Wiers et al., 2002, 2005). Here, negative alcohol associations were replicated with unipolar IATs that contrasted negative words with neutral words. Moreover, testing alcohol associations in a unipolar format also revealed positive alcohol associations, demonstrating that implicit alcohol associations are ambivalent. Further, as hypothesized, the labeling of the target category (alcohol or beer) and the choice of the contrast category (animals or sodas) influenced the strength of negative alcohol associations. Nevertheless, in all IAT versions, strong negative alcohol associations emerged, suggesting that they reflect something 'real' in the memory of drinkers rather than an IAT artifact. Possibly, negative implicit alcohol associations rise with alcohol use until some threshold is reached after which they begin to influence behavior (Jones \& McMahon, 1996). Here, however, no support was found for an increase in implicit negative alcohol associations with increased alcohol use. Alternatively, implicit negative alcohol-related cognitions could stem from early (negative) alcohol experiences and explicit positive alcohol-related cognitions from recent (positive) experiences. The present pattern of results, however, was not fully consistent with this hypothesis, implying that the distinction between implicit and explicit cognitions and their origins is not as clearcut for alcohol as for smoking (cf. Rudman, 2004). Hence, the question remains what these robust negative alcohol associations really mean. A remaining possibility is that they reflect negative reinforcement expectancies. Since targets and attributes alternate in the IAT, targets both precede and follow attributes. An observed association between alcohol and an attribute can therefore reflect both an association with an expected outcome (e.g., alcohol-positive: alcohol causes a positive mood) or with an antecedent mood state (e.g., positive-alcohol: in a positive mood, drink alcohol).

Second, assessing alcohol associations in a unipolar format also revealed implicit alcohol associations with both arousal and sedation. Importantly, arousal associations predicted alcohol use while sedation associations predicted alcohol-related problems, above explicit measures. Further, implicit arousal associations were paralleled by explicit arousal expectancies and both were predictive of alcohol use. These results both replicate and extend previous findings (e.g., Wiers et al. 2002, 2005) and provide additional evidence for the development of arousal associations with drinking experience. The present 
results may reflect the operation of a "wanting" (sensitized arousal) system that becomes sensitized with repeated alcohol use and shows a progressive increase in the psychomotor activating response to alcohol (Robinson \& Berridge, 1993, 2003). Alcohol-related problems, on the other hand, were best predicted by implicit sedation associations, which could be related to research demonstrating that negative reinforcement motivations are strong predictors of alcohol-related problems (e.g., Cooper, Frone, Russell, \& Mudar, 1995). Together, these findings suggest that especially drinkers who consume alcohol to cope with negative affect are at risk of experiencing alcohol-related problems and developing a maladaptive drinking pattern.

A second study examined whether the present findings with unipolar IATs could be explained by figure-ground asymmetries. This was generally not the case, and certainly not for the alcohol-arousal associations. In sum, the present results underscore the usefulness of assessing alcohol associations in a unipolar fashion, support an explanation of implicit alcohol associations as meaningful memory constructs and refute artifact explanations in terms of target bipolarity, label effects and figure-ground effects.

\section{Strengths and Limitations of the IAT and Issues for Future Research}

The IAT is useful for examining implicit associations but it does not come without limitations (cf. De Houwer, 2002). The present research showed that some of these limitations are not necessarily insuperable by demonstrating that the IAT, with minor revisions, can be used to assess ambivalent alcohol associations. However, it should be noted that the unipolar alcohol-IATs that were used here showed smaller internal consistencies than those reported for the bipolar alcohol-IAT (about .80; Wiers et al., 2005). Further, careful consideration should be given to the choice of the contrast target category. The present results show that contrasting alcohol with either soda or animals makes little difference. However, it should be noted that soda is a natural contrast to alcohol whereas the category animals is not. When a target category does not have a clear contrast (e.g., smoking), using such a neutral contrast may be useful, yet difficult (cf. Swanson, Rudman, \& Greenwald, 2001). This issue should be examined in future studies, but in the meantime, testing alternative association measures that do not require two contrasting target concepts could be a useful strategy (e.g., De Houwer, 2003; Nosek \& Banaji, 2001). Finally, using visual search tasks as a supplement to the IAT is recommended to examine confounding due to salience asymmetries.

In conclusion, implicit alcohol associations can be assessed in a meaningful way with unipolar IATs. This research strategy can be used in future studies on the role of implicit and explicit cognitions in the etiology and maintenance of alcohol abuse. Hopefully, further research on assessment issues of implicit alcohol-related cognitions will lead to a better understanding of automatic processes in addictive behaviors and their treatment. 


\section{APPENDIX}

\section{IAT Target Stimuli}

Alcohol: wine, Bacardi, whisky, Heineken, Hoegaarden, Amstel (the last three are beer brands).

Beer: trappist, pint, Jupiler, Heineken, Hoegaarden, Amstel

Soda: Spa (sparkling water), Fanta, Coca Cola, Sprite, apple juice, sinas (lemonade)

Animals: cricket, duck, lizard, rhino, ostrich, hedgehog

\section{IAT Valence Attribute Stimuli}

Pleasant: love, sunshine, warmth, peace, hug, rainbow

Unpleasant: war, depression, pain, fight, disease, sorrow

Neutral valence 1: paper, circle, ballpoint, factory, truck, magnet

Neutral valence 2: letter, square, page, machine, scissors, window

\section{IAT Arousal/Sedation Attribute Stimuli}

Active: talkative, jovial, restless, alert, unrestrained, rambunctious

Quiet: silent, listless, sleepy, passive, relaxed, calm

Neutral arousal 1: constant, wide, brown, digital, recent, usual

Neutral arousal 2: oval, compact, related, central, daily, steep 


$$
\text { D) }
$$




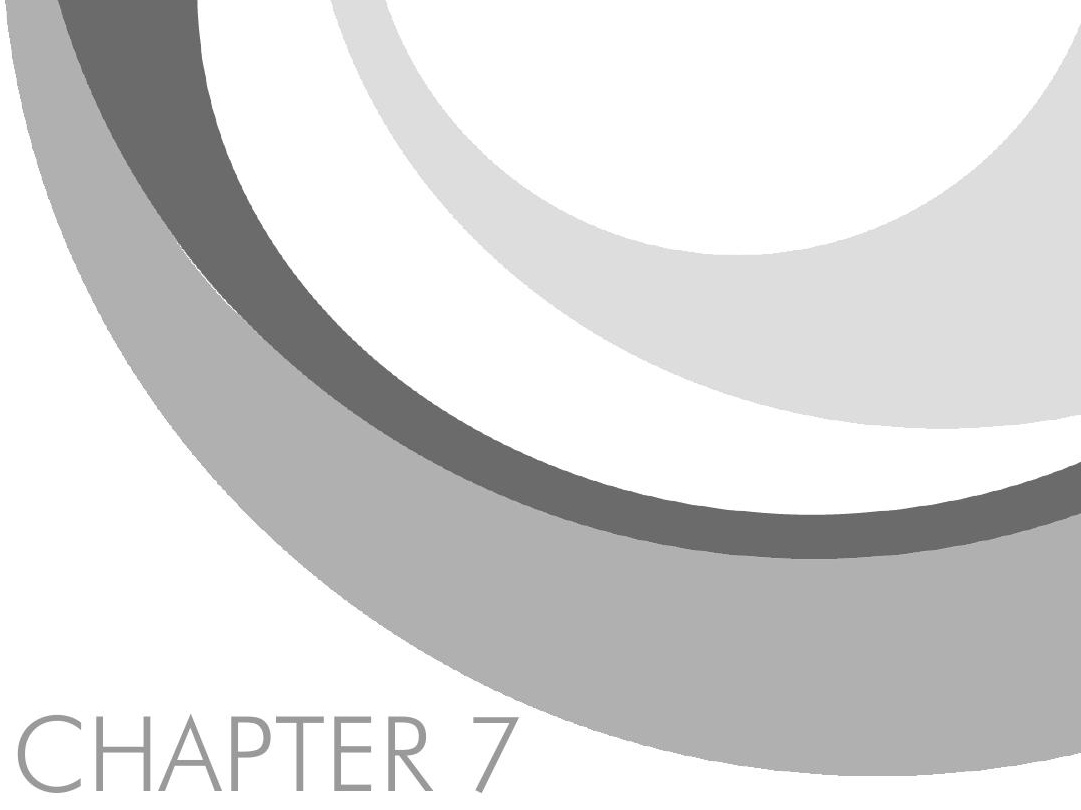

\section{Single-Target Implicit Preferences for Beer as Determinants of Consumption}

Submitted for publication as: Houben, K., \& Wiers, R. W. (2006). Single-target implicit preferences for beer as determinants of consumption. 


\begin{abstract}
Previous studies demonstrated that implicit positive attitudes toward alcohol, assessed with unipolar Implicit Association Tests (IATs), are involved in drinking behavior while implicit negative alcohol attitudes are not. However, because the IAT is a relative measure, the present study aimed at replicating these findings with non-relative unipolar Single TargetIATs (ST-IATs). Further, ST-IATs were performed with either alcohol or beer as the target concept to examine whether previous results could be due to extrapersonal contamination of the IAT caused by cultural connotations of the concept alcohol. It was expected that beer ST-IATs would be less affected by extrapersonal contamination since all participants included in the study were regular beer drinkers. Consistent with previous findings, both alcohol and beer ST-IATs supported the coexistence of implicit positive and implicit negative alcohol-related attitudes. Importantly, positive beer ST-IAT scores were related to drinking behavior, while positive alcohol ST-IAT scores were not. Hence, the present results suggest that the positive beer ST-IAT assessed more personally relevant implicit attitudes that play a role in drinking behavior compared to the positive alcohol ST-IAT.
\end{abstract}

\title{
INTRODUCTION
}

The past decade, there has been a surge of interest in implicit cognitive processes involved in addictive behaviors. This research interest was primarily fueled by the advent of indirect measures that are designed to minimize controlled responding. Additionally, research suggests that indirect measures can assess implicit cognitions that influence behavior automatically (e.g. Gawronski, Hofmann, \& Wilbur, 2006) and can, therefore, provide unique insights into the cognitive processes underlying addictive behaviors. Counter intuitively, several studies using the Implicit Association Test (IAT; Greenwald, McGhee, \& Schwartz, 1998) to study implicit alcohol-related cognitive processes demonstrated that heavy (and light) drinkers display implicit negative attitudes toward alcohol, suggesting that implicit alcohol attitudes are not a driving force behind drinking behavior (e.g., De Houwer, Crombez, Koster, \& De Beul, 2004; Wiers, van Woerden, Smulders, \& de Jong, 2002). However, the bipolarity of the evaluative dimension in the IAT (i.e., positive vs. negative) could have prevented these studies from finding behavior-relevant implicit alcohol attitudes, given that attitudes toward alcohol could be highly ambivalent. In line with this idea, studies using IATs with the evaluative categories in a unipolar format (e.g., positive vs. neutral), demonstrated that implicit positive alcohol attitudes are an important determinant of drinking behavior (e.g., Houben \& Wiers, 2006c; Jajodia \& Earleywine, 2003). Implicit negative alcohol attitudes, on the other hand, did not show a relationship with drinking behavior. 
However, the relationship between IAT scores and drinking behavior could have been deflated in previous studies due to procedural limitations of the IAT. First, given its relative nature, the alcohol-IAT can reflect both implicit alcohol attitudes and/or implicit soda attitudes. Therefore, the present study aimed to replicate previous findings using the non-relative Single-Target IAT (ST-IAT; Wigboldus, Holland, \& van Knippenberg, 2006) which is structurally similar to the IAT with the difference that it measures implicit attitudes toward single target concepts. Here, we used unipolar ST-IATs to assess implicit alcohol attitudes. Further, research suggests that alcohol-IAT scores could at least partly reflect extrapersonal associations with alcohol that are irrelevant to behavior (Houben \& Wiers, 2006b). Therefore, unipolar ST-IATs were used to measure implicit attitudes toward alcohol or beer. Since only participants who regularly drank beer were included in the study, it was expected that unipolar beer ST-IATs would be less contaminated by extrapersonal associations and would, thus, correlate more strongly with drinking behavior compared to unipolar alcohol ST-IATs.

\section{METHOD}

Participants were 48 students who drank beer on a regular basis (24 male; mean age $=$ 20.25 years, $S D=2.23)$. Alcohol use was measured with the timeline follow-back method (Sobell \& Sobell, 1990). In the week prior to the study, participants on average consumed $16.67(S D=13.09)$ standard servings, including $12.71(S D=12.59)$ beer consumptions. Alcohol-related problems were assessed with the 18-item Rutgers Alcohol Problem Index (RAPI; White \& Labouvie, 1989; mean item score $=.46, \mathrm{SD}=.27$ ) and the Alcohol Use Disorder Identification Test (AUDIT; Saunders, Aasland, Babor, De la Fuente, \& Grant, 1993; mean score $=10.04, S D=3.25$ ). Participants were randomly divided into an alcohol condition or a beer condition. Implicit attitudes toward alcohol or beer (depending on condition) were assessed with a positive and a negative ST-IAT. All stimuli are listed in the Appendix. The positive and negative ST-IATs consisted of 4 blocks (see Table 1) and were modeled after the ST-IAT by Wigboldus et al. (2006). Explicit expectancies with respect to alcohol or beer (depending on condition) were assessed with an expectancy questionnaire. Participants indicated on a Visual Analogue Scale (VAS) how much they agreed $(0=$ completely disagree, $100=$ completely agree) with 6 positive and 6 negative expectancy items. Each item consisted of the statement: "After drinking alcohol (beer), I feel . . .", which was completed with the following words: pleasant, happy, sociable, friendly, enjoyable, and likable for the positive expectancy items, and unhappy, depressive, sad, lonely, moody, and down for the negative expectancy items. Explicit attitudes toward alcohol or beer (depending on condition) were assessed with an attitude questionnaire and a feeling thermometer. The attitude questionnaire consisted of 4 semantic differentials: Participants indicated on a VAS how much they considered drinking 
alcohol (beer) to be unpleasant-pleasant, boring-fun, bad-good, and stupid-smart (the first two items formed an affective attitude component while the last two items formed a cognitive component). On the feeling thermometer, participants indicated how favorable they felt toward alcohol (beer) $(0=$ very cold, $100=$ very warm).

Table 1

Overview of ST-IAT phases.

\begin{tabular}{lllll}
\hline Block & Trials & Function & Left key & Right key \\
\hline 1 & 24 & Attribute practice & pleasant & neutral \\
2 & 24 & Compatible combination task & alcohol & \\
& & & pleasant & neutral \\
3 & 24 & Reversed attribute practice & neutral & pleasant \\
4 & 24 & Incompatible combination task & alcohol & \\
& & & neutral & pleasant \\
\hline
\end{tabular}

Note. Blocks are shown for the positive alcohol ST-IAT. In the beer condition, the alcohol target category was replaced by the beer target category. To keep the number of right and left responses equal, stimuli of the two categories that shared a response were presented twice, while stimuli of the unpaired neutral category were presented four times. Blocks 1 to 4 were repeated in the negative ST-IAT with the attribute categories 'unpleasant' and 'neutral'. The order of the positive and negative ST-IATs and the response assignment of the target category were balanced across participants. The response assignment of the attribute categories was counterbalanced so that half the participants always received the compatible combination task before the incompatible combination task whereas the other half received the reversed task order.

\section{RESULTS}

ST-IAT effects were calculated with the D600 scoring algorithm (Greenwald, Nosek, \& Banaji, 2003) so that higher ST-IAT scores indicate stronger evaluative (positive or negative) associations with alcohol or beer. No difference emerged between the alcohol condition and the beer condition on positive ST-IAT scores, $t(46)=.29, p=.773$, or negative ST-IAT scores, $t(46)=.45, p=.653$. Results showed implicit positive attitudes toward both alcohol, $t(23)=4.57, p<.001$, and beer, $t(23)=5.34, p<.001$, as well as implicit negative attitudes toward both alcohol, $t(23)=5.87, p<.001$, and beer, $t(23)$ $=4.84, p<.001$. With respect to explicit measures, results indicated that participants, to the same extent, expected positive effects, but not negative effects, from drinking alcohol and beer and that attitudes toward alcohol and soda were equally favorable (see Table 2). Except for a correlation between positive ST-IAT scores and cognitive attitude scores, in the alcohol condition, none of the correlations between implicit and explicit cognitions reached significance (see Table $3 \& 4$ ). 
Table 2

Mean values for explicit positive and negative expectancies, the affective and cognitive attitude component, and the feeling thermometer, separately for the alcohol and beer conditions.

\begin{tabular}{|c|c|c|c|c|c|c|}
\hline & \multicolumn{2}{|c|}{ Alcohol } & \multicolumn{2}{|c|}{ Beer } & \multirow[b]{2}{*}{$t$} & \multirow[b]{2}{*}{$p$} \\
\hline & M & $S D$ & M & $S D$ & & \\
\hline Positive expectancies & 76.56 & 13.49 & 73.28 & 13.66 & .83 & .412 \\
\hline Negative expectancies & 18.65 & 13.81 & 17.85 & 15.20 & .19 & .851 \\
\hline Affective attitude & 86.06 & 11.76 & 79.33 & 22.98 & 1.27 & .210 \\
\hline Cognitive attitude & 47.00 & 14.48 & 47.11 & 19.38 & -.02 & .983 \\
\hline Feeling thermometer & 72.08 & 10.21 & 65.00 & 17.97 & 1.67 & .102 \\
\hline
\end{tabular}

An alcohol use estimate was calculated as the mean of log-transformed average alcohol consumption in the past week and log-transformed average weekly alcohol consumption. An estimate of alcohol-related problems was computed as the mean of z-transformed RAPI and AUDIT sum scores. Alcohol use and alcohol-related problems were significantly correlated, $r=$ $.60, p=.002$. In the alcohol condition, explicit alcohol-related cognitions, but not ST-IAT scores, were significantly related to drinking behavior. Specifically, participants who held stronger positive attitudes toward alcohol and weaker negative expectancies consumed more alcohol (see Table 3). In the beer condition, alcohol use was related to both explicit beer-related cognitions and positive ST-IAT scores, indicating that participants who displayed stronger positive attitudes toward beer, both implicitly and explicitly, consumed more alcohol (see Table 4).

Table 3

Correlations between ST-IAT scores, explicit expectancies and attitudes, and alcohol use and alcohol-related problems in the alcohol condition.

\begin{tabular}{|c|c|c|c|c|c|c|c|}
\hline \multirow[t]{2}{*}{ Variable } & \multicolumn{2}{|c|}{ Alcohol ST-IAT } & \multicolumn{2}{|c|}{ Alcohol expectancies } & \multicolumn{2}{|c|}{ Alcohol attitude } & \multirow{2}{*}{$\begin{array}{l}\text { Feelings } \\
\text { toward } \\
\text { alcohol }\end{array}$} \\
\hline & Positive & Negative & Positive & Negative & Affective & Cognitive & \\
\hline Negative alcohol ST-IAT & .27 & - & & & & & \\
\hline Positive alcohol expectancies & .00 & -.16 & - & & & & \\
\hline Negative alcohol expectancies & -.18 & -.15 & $-.62^{* *}$ & - & & & \\
\hline Affective alcohol attitude & .12 & -.22 & $.46^{*}$ & $-.57^{* *}$ & - & & \\
\hline Cognitive alcohol attitude & $.51^{*}$ & .23 & -.08 & -.21 & .26 & - & \\
\hline Feelings toward alcohol & .34 & .01 & $.42^{*}$ & $-.51^{*}$ & $.69^{* *}$ & .39 & - \\
\hline Alcohol use & -.05 & -.16 & .18 & $-.54^{* *}$ & $.63^{* *}$ & .17 & $.43^{*}$ \\
\hline Alcohol-related problems & -.24 & -.04 & .18 & -.19 & $.47^{*}$ & -.07 & .40 \\
\hline
\end{tabular}


Table 4

Correlations between ST-IAT scores, explicit expectancies and attitudes, and alcohol use and alcohol-related problems in the beer condition.

\begin{tabular}{|c|c|c|c|c|c|c|c|}
\hline \multirow[t]{2}{*}{ Variable } & \multicolumn{2}{|c|}{ Beer ST-IAT } & \multicolumn{2}{|c|}{ Beer expectancies } & \multicolumn{2}{|c|}{ Beer attitude } & \multirow{2}{*}{$\begin{array}{l}\text { Feelings } \\
\text { toward beer }\end{array}$} \\
\hline & Positive & Negative & Positive & Negative & Affective & Cognitive & \\
\hline Negative beer ST-IAT & .12 & - & & & & & \\
\hline Positive beer expectancies & -.03 & .06 & - & & & & \\
\hline Negative beer expectancies & -.15 & -.06 & -.20 & - & & & \\
\hline Affective beer attitude & .18 & .05 & $.42^{*}$ & .05 & - & & \\
\hline Cognitive beer attitude & .15 & .26 & .25 & .08 & $.63^{* *}$ & - & \\
\hline Feelings toward beer & .32 & .12 & $.56^{* *}$ & -.07 & $.72^{* *}$ & $.44^{*}$ & - \\
\hline Alcohol use & $.42^{*}$ & .23 & -.02 & -.11 & $.47^{*}$ & .37 & .25 \\
\hline Alcohol-related problems & .19 & .24 & .04 & -.02 & .20 & .27 & .03 \\
\hline
\end{tabular}

\section{DISCUSSION}

Previous studies using unipolar IATs suggest that implicit positive attitudes toward alcohol play a role in drinking behavior whereas implicit negative alcohol attitudes do not (e.g., Houben \& Wiers, 2006c; Jajodia \& Earleywine, 2003). The present study, we measured single implicit attitudes toward alcohol or beer with unipolar ST-IATs to examine to what extent previous findings were affected by the relative nature of unipolar IATs as well as extrapersonal contamination of unipolar IAT scores. Specifically, it was expected that beer ST-IATs would be less affected by extrapersonal contamination and would, thus, assess behavior-relevant implicit attitudes to a greater extent compared to alcohol ST-IATs, since all participants were regular beer drinkers. Results showed no indication that alcohol STIAT scores and beer ST-IAT scores were differentially affected by extrapersonal contamination and the coexistence of implicit positive attitudes and implicit negative attitudes toward both alcohol and beer was demonstrated. Consistent with the hypothesis, however, positive beer ST-IAT scores were related to alcohol use while positive alcohol STIAT scores were not. Negative alcohol and beer ST-IAT scores were unrelated to drinking behavior. Hence, the present results replicate previous findings with the unipolar IAT, indicating that implicit positive alcohol-related attitudes, but not implicit negative attitudes, play a role in drinking behavior (e.g., Houben \& Wiers, 2006c; Jajodia \& Earleywine, 2003) and show that these results were not due to the relative nature of the IAT.

Importantly, the current results also extend previous findings by demonstrating that, in the present sample, beer ST-IATs, but not alcohol ST-IATs, assessed personally relevant implicit attitudes that were also important determinants of drinking behavior. Importantly, 
participants' alcohol consumption was almost completely confined to drinking beer rather than different kinds of alcoholic drinks. Thus, these findings imply that, in the (ST-)IAT, target categories should be as specific as possible given the behavior that needs to be explained. Consistent with this implication, Fadardi, Cox, and Klinger (2006) have argued that it is questionable to what extent an IAT that presents standard stimuli can capture participants' personal implicit attitudes, since standard stimuli will probably differ in the degree to which they are both known and liked across participants. While the current findings indicate that it is useful to customize the target category of interest, it might be even more valuable to personalize individual target stimuli as well, in order to make sure that (ST-)IAT scores reflect personally endorsed implicit attitudes that are relevant to behavior. 


\section{APPENDIX}

\section{ST-IAT Target Stimuli}

Alcohol category (label 'alcohol'): wine, Bacardi, whisky, Heineken, Hoegaarden, Amstel (the last three are beer brands).

Beer category (label 'beer'): Trappist, Pils, Jupiler, Heineken, Hoegaarden, Amstel

\section{ST-IAT Attribute Stimuli}

Positive category (label 'pleasant'): love, sunshine, warmth, peace, hug, rainbow Negative category (label 'unpleasant'): war, depression, pain, fight, disease, sorrow Neutral category 1 (label 'neutral'): paper, circle, ballpoint, factory, truck, magnet Neutral category 2 (label 'neutral'): letter, square, page, machine, scissors, window

The positive and negative attribute categories were paired with the neutral attribute categories in balanced order. The (Dutch) attribute categories were matched on familiarity, arousal, and number of syllables. 



$$
\text { D) }
$$




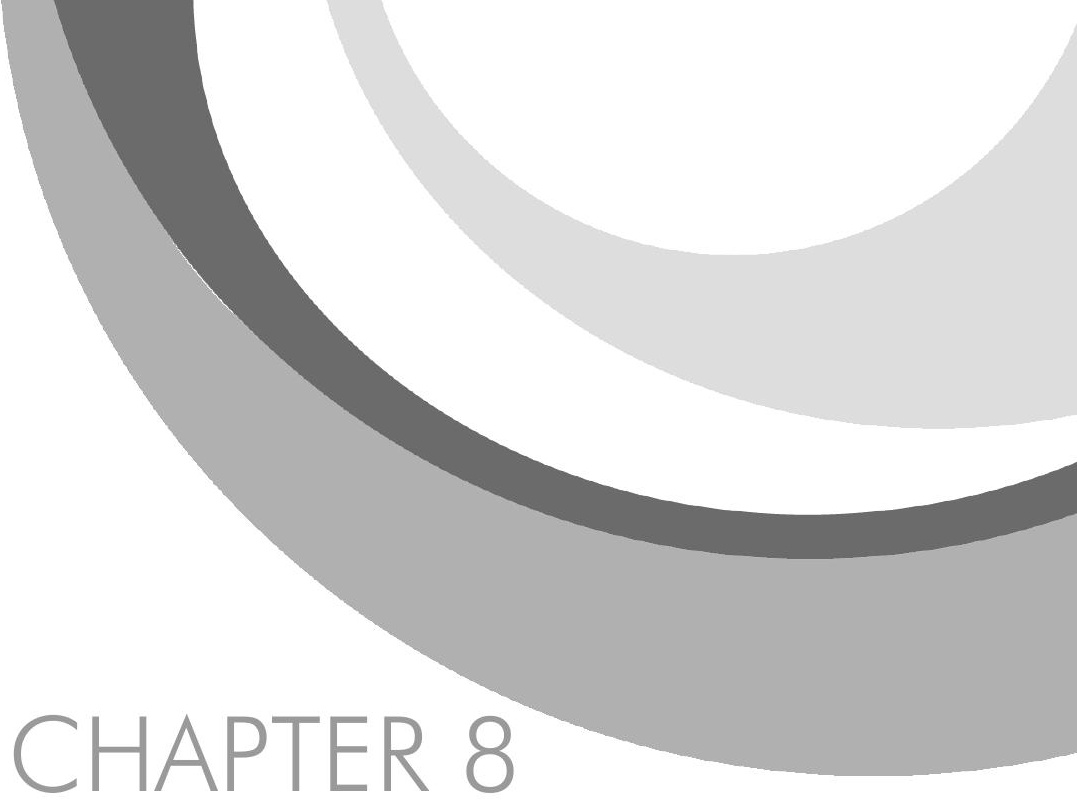

\section{Implicit Positive Alcohol Attitudes as Determinants of Alcohol Consumption: Beyond the Soda Contrast in the IAT} attitudes as determinants of alcohol consumption: Beyond the soda contrast in the IAT. 


\begin{abstract}
Research using unipolar Implicit Association Tests (IATs), has demonstrated that implicit positive alcohol attitudes but not implicit negative alcohol attitudes are involved in drinking behavior. However, the relative nature of the IAT with respect to target concepts (i.e., alcohol vs. soda) obscures the interpretation of IAT scores and their relationship to behavior. Here, unipolar alcohol-soda IATs were contrasted with unipolar Single Target IATs (ST-IAT) for alcohol alone. While the alcohol-soda IATs yielded only implicit negative alcohol attitudes, both positive and negative implicit alcohol attitudes emerged in the alcohol ST-IATs. Moreover, alcohol-soda IATs were uncorrelated with both alcohol ST-IATs and unipolar soda ST-IATs, suggesting that single implicit attitudes assessed with the STIAT are qualitatively different from implicit attitudes assessed with the IAT. Importantly, implicit positive alcohol attitudes assessed with both the alcohol-soda IAT and the alcohol ST-IAT predicted alcohol use above explicit alcohol-related cognitions. Together, the present findings demonstrate that the unipolar IAT which measures implicit attitudes toward alcohol relative to soda, and the ST-IAT which assesses single implicit attitudes toward alcohol, provide unique contributions to the prediction of drinking behavior.
\end{abstract}

\title{
INTRODUCTION
}

Recent research suggests that indirect measures can be used to register implicit cognitions that are activated automatically and which can influence behavior outside conscious awareness (e.g., Gawronski, Hofmann, \& Wilbur, 2006). Indirect measures can thus provide unique insights in cognitive processes involved in addictive behaviors such as alcohol use and abuse since these underlying processes are likely to develop automaticity with increased substance use (e.g., Evans \& Coventry, 2006; Stacy, 1997). Many studies that have addressed the role of implicit cognitions involved in alcohol use and abuse have done so using the Implicit Association Test (Greenwald, McGhee, \& Schwartz, 1998). The reason that the IAT is the most commonly used indirect measure is that it is easy to administer, produces large and robust effects and has been shown to provide a reliable and valid measure of implicit cognitions (e.g., Hofmann, Gawronski, Gschwendner, Le, \& Schmitt, 2005; Nosek, Greenwald, \& Banaji, 2006; Poehlman, Uhlmann, Greenwald, \& Banaji, 2005). The underlying logic of the IAT is that it should be easier to classify stimuli into two target concepts (e.g., flowers and insects) and two attribute categories (e.g., positive and negative) when associated concepts are assigned to the same response (e.g., flowers + positive and insects + negative) than when associated concepts are assigned to different responses (e.g., flowers + negative and insects + positive). 
Evidence from the first studies that examined implicit alcohol-related cognitions using the IAT suggested that implicit evaluative associations with alcohol, or implicit attitudes, did not appear to be involved in drinking behavior. More specifically, Wiers, van Woerden, Smulders, \& de Jong (2002) demonstrated that both light and heavy drinkers more easily paired alcohol with a negative evaluative category and soda with a positive evaluative category than vice versa. Differentiation between light and heavy drinkers, however, was established with a second IAT that assessed implicit associations between alcohol and arousal: Heavy drinkers were faster when alcohol shared a response with arousal and soda with sedation than in the reversed combination. Importantly light drinkers did not show this IAT effect. Moreover, these findings were replicated both in a sample of heavy drinkers (Wiers, van de Luitgaarden, van den Wildenberg, \& Smulders, 2005) as well as in a sample of patients undergoing treatment (De Houwer, Crombez, Koster, \& De Beul, 2004). Hence, in line with the incentive-sensitization account (Robinson \& Berridge, 1993), Wiers et al. (2002) suggested that implicit attitudes toward alcohol, or "liking", were not an important determinant of drinking behavior, at least not as important as implicit alcohol associations with arousal which presumably reflected the operation of a sensitized "wanting" system.

However, it is important to note that the standard IAT procedure measures the strength of the target concepts with two evaluative categories, positive and negative. Consequently, IAT effects only indicate whether target concepts are more associated with one evaluative category relative to the other evaluative category. As such, this bipolarity of the evaluative dimension potentially precludes the assessment of behavior-relevant implicit attitudes toward alcohol given that attitudes toward alcohol can be highly ambivalent (e.g., Conner \& Sparks, 2002). In line with this idea, recent studies that have eliminated the bipolarity of the evaluative dimension in the alcohol-soda IAT, suggest a more important role of implicit attitudes in alcohol use and abuse. Specifically, in unipolar IATs, both evaluative categories are tested against neutral categories so that a positive unipolar IAT can assess the strength of implicit positive attitudes toward the target concepts and a negative unipolar IAT can measure the strength of implicit negative attitudes toward the target concepts. Importantly, results with unipolar alcohol-soda IATs demonstrated that implicit positive attitudes toward alcohol were at least moderately related to drinking behavior while implicit negative attitudes toward alcohol were not (e.g., Houben \& Wiers, 2006c; Jajodia \& Earleywine, 2003). Hence, these findings suggest that implicit positive attitudes play a role in alcohol use and abuse, but that they are overshadowed by strong implicit negative evaluative associations with alcohol in a standard bipolar IAT. In addition, these strong implicit negative evaluative associations with alcohol could reflect extrapersonal associations with alcohol that are not part of one's personal attitudes toward alcohol and that are irrelevant to behavior (e.g., Houben \& Wiers, 2006b).

Although the available evidence seems to support the role of implicit positive attitudes toward alcohol in drinking behavior and not of implicit negative alcohol attitudes, 
it should be noted that the IAT procedure has another important limitation that compromises this interpretation of alcohol-IAT effects and their relationship with behavior. Specifically, the IAT procedure cannot reveal implicit evaluative associations with single target concepts such as alcohol, but is instead limited to measuring the relative strengths of pairs of implicit associations. While this relative nature of the IAT is not a problem for most research questions as many attitude concepts have a complementary category that can be used as a contrast in the IAT (e.g., black vs. white, old vs. young, self vs. other), it is a problem when one is interested in implicit associations with a single target concept or when the target concept of interest does not have a natural complement. More importantly, the relative nature of the IAT obscures the interpretation of IAT scores. For example, with respect to the alcohol-soda IAT, IAT scores can reflect both implicit associations with alcohol and/or implicit associations with soda. Hence, in case of the negative unipolar alcohol-soda IAT, high IAT scores could indicate strong implicit negative alcohol attitudes, but also weak negative attitudes toward soda in which case it would not be surprising that these IAT scores do not relate to alcohol consumption. Further, with respect to the positive unipolar alcohol-soda IAT, this ambiguity of IAT scores potentially deflates the validity of the IAT as a measure of implicit positive alcohol associations well as its predictive validity with respect to drinking behavior. Consequently, there is clearly a need for indirect measures that can assess implicit attitudes toward single target concepts. The current study examines the application of such a non-relative indirect measure: the Single Target IAT (ST-IAT; Wigboldus, Holland, \& van Knippenberg, 2006).

The ST-IAT is structurally similar to the IAT with the difference that only one target category is presented during the task. Thus, unlike the IAT, the ST-IAT measures implicit associations with single target concepts, without the need of a complementary contrast category. Consequently, it was hypothesized that an alcohol ST-IAT could provide a more specific measure of implicit alcohol attitudes than the alcohol-soda IAT. It should be noted, however, that the ST-IAT procedure, like the standard IAT procedure, is limited in the sense that evaluative associations are assessed in a bipolar format with respect to the attribute dimension. In the present study, the ST-IAT was therefore presented in a unipolar format similar to what was previously done successfully with the IAT (e.g., Houben \& Wiers, 2006c; Jajodia \& Earleywine, 2003). The goal of the present study was to examine whether unipolar alcohol ST-IATs measure qualitatively different implicit alcohol attitudes compared to unipolar alcohol-soda IATs. To this end, we assessed implicit alcohol attitudes with both a positive and a negative alcohol-soda IAT and with both a positive and a negative alcohol ST-IAT. In this way, we were able to examine whether assessing single implicit alcohol attitudes yields different results compared to measuring implicit attitudes toward alcohol relative to soda. Moreover, if assessing implicit alcohol attitudes with the ST-IAT yields additional information compared to measuring implicit alcohol attitudes with the alcohol-soda IAT, alcohol ST-IAT scores should predict alcohol-related behavior above alcohol-soda IAT scores. In addition, we also measured implicit soda attitudes with a 
positive and a negative soda ST-IAT to examine the contributions of both single implicit alcohol attitudes and single implicit soda attitudes to IAT scores. Finally, we also examined the relationship of all indirect measures with explicit alcohol-related and soda-related expectancies and attitudes.

\section{METHOD}

\section{Participants}

Sixty-two female students of Maastricht University participated in this study in return for course credit or a gift certificate of $10 €$. On average, participants consumed 6.71 (SD = 7.00) European standard alcoholic drinks ${ }^{18}$ per week. On the 18-items version of the Rutgers Alcohol Problem Index (RAPI; White \& Labouvie, 2000), participants had an average item score of .26 (SD = .34), while participants' mean score on the Alcohol Use Disorder Identification Test (AUDIT; Saunders, Aasland, Babor, De la Fuente, \& Grant, 1993) was 7.11 (SD = 4.52).

\section{Materials and Measures}

Alcohol Use. Alcohol use was measured through a self-report questionnaire (Wiers, Hoogeveen, Sergeant \& Gunning, 1997) based on the timeline follow-back method (Sobell \& Sobell, 1990). Participants were asked to indicate how many drinks of different types of alcoholic beverages they consumed during each day of the past week, and for each day of the week, how many drinks they typically consumed on this day of the week.

Alcohol-Related Problems. Alcohol-related problems were assessed with the RAPI and with the AUDIT. The RAPI described 18 alcohol-related problems or situations for which participants indicated how often they experienced these situations on a 5-point Likert scale $(0=$ never, $4=$ very often) (Cronbach $\alpha=.86)$. The AUDIT consisted of 10 questions in a multiple choice format. The first three questions were related to alcohol use, the other seven to alcohol-related problems ( $\alpha=.83$ ).

Implicit Measures of Alcohol and Soda Attitudes. In both the positive IAT and the negative IAT, two target categories were presented, the first consisting of 5 alcoholic drinks (label 'alcohol') and the other of 5 sodas (label 'soda'). Further, the alcohol target category was also presented in both the positive and the negative alcohol ST-IAT, while the soda target category was also used in both the positive and the negative soda ST-IAT. The

${ }^{18}$ A standard European alcoholic drink contains somewhat less alcohol than a standard English or American alcoholic drink: 10-12g vs. $14 \mathrm{~g}$. 
two (Dutch) target categories were matched on number of syllables. In the positive IAT as well as in the positive alcohol ST-IAT and the positive soda ST-IAT, a positive attribute category (label 'pleasant') was presented, whereas in the negative IAT and in both the negative alcohol ST-IAT and the soda ST-IAT, a negative attribute category (label 'unpleasant') was presented. Further, the positive and negative attribute categories were paired with 4 neutral attribute categories (label 'neutral') in balanced order ${ }^{19}$. All attribute categories were matched on number of syllables. All stimuli are listed in the Appendix.

All tasks were programmed in ERTS 3.18 (Beringer, 1996). The positive IAT and the negative IAT followed the standard IAT procedure (see Greenwald et al., 1998) with the modification that both IATs were presented so that they appeared to form one single task. Participants always first practiced the target discrimination because it remained constant across the positive IAT and negative IAT. Next, participants completed the positive IAT and the negative IAT in balanced order, each consisting of 4 blocks. In each block, all stimuli of the respective categories were presented twice. The alcohol and soda ST-IATs followed the procedure of the ST-IAT developed by Wigboldus et al. (2005). However, as in the IAT, participants performed the positive and negative ST-IAT in balanced order with each STIAT consisting of 4 blocks. Further, during attribute practice blocks, all stimuli were presented twice whereas in the combination blocks, stimuli of the two categories that shared a response were presented twice, while stimuli of the unpaired neutral category were presented four times in order to keep the number of right and left responses equal. The assignment of the alcohol category and the soda category to the response keys was balanced across participants and remained constant across all IAT and ST-IAT versions. Further, the response assignment of the attribute categories was also counterbalanced so that half the participants in each task performed the compatible combination task before the incompatible combination task whereas the other half always performed the incompatible combination task before the compatible combination task. An overview of IAT and ST-IAT blocks is shown in Table 1.

During all tasks, target and attribute stimuli were presented in the middle of the computer screen, in black against a grey background. Instructions were presented before each task. During the task, the labels of the categories assigned to the left and right response key were presented in the corresponding upper corners of the computer screen. Stimuli remained on screen until a response was given. The intertrialinterval was $250 \mathrm{~ms}$. Feedback was presented in red beneath the stimuli after an incorrect response ('wrong'),

\footnotetext{
${ }^{19}$ For half the participants, two neutral categories that consisted of heterogeneous sets of neutral words were paired in balanced order with the positive and negative category, whereas for the other half of the participants the positive and negative category were paired in balanced order with two neutral categories that consisted of homogeneous sets of neutral words which were all synonyms of the word 'neutral'. The composition (heterogeneous or homogenous) of the neutral categories did not influence any of the results reported here.
} 
and when responses were too fast $(<300 \mathrm{~ms}$; 'too fast') or too slow (> $3000 \mathrm{~ms}$; 'too slow').

Table 1

Overview of IAT and ST-IAT phases.

\begin{tabular}{|c|c|c|c|c|c|c|c|c|c|}
\hline \multicolumn{5}{|c|}{ IAT } & \multicolumn{5}{|c|}{ ST-IAT } \\
\hline Block & Trials & Function & Left key & Right key & Block & Trials & Function & Left key & Right key \\
\hline 1 & 24 & Target practice & alcohol & soda & & & & & \\
\hline 2 & 24 & Attribute practice & pleasant & neutral & 1 & 24 & Attribute practice & pleasant & neutral \\
\hline $3 a$ & 24 & $\begin{array}{l}\text { Compatible } \\
\text { combination } \\
\text { practice }\end{array}$ & $\begin{array}{l}\text { alcohol } \\
\text { pleasant }\end{array}$ & $\begin{array}{l}\text { soda } \\
\text { neutral }\end{array}$ & $2 a$ & 24 & $\begin{array}{l}\text { Compatible } \\
\text { combination } \\
\text { practice }\end{array}$ & $\begin{array}{l}\text { alcohol } \\
\text { pleasant }\end{array}$ & neutral \\
\hline $3 b$ & 24 & $\begin{array}{l}\text { Compatible } \\
\text { combination } \\
\text { test }\end{array}$ & $\begin{array}{l}\text { alcohol } \\
\text { pleasant }\end{array}$ & $\begin{array}{l}\text { soda } \\
\text { neutral }\end{array}$ & $2 b$ & 24 & $\begin{array}{l}\text { Compatible } \\
\text { combination } \\
\text { test }\end{array}$ & $\begin{array}{l}\text { alcohol } \\
\text { pleasant }\end{array}$ & neutral \\
\hline 4 & 24 & $\begin{array}{l}\text { Reversed attribute } \\
\text { practice }\end{array}$ & neutral & pleasant & 3 & 24 & $\begin{array}{l}\text { Reversed attribute } \\
\text { practice }\end{array}$ & neutral & pleasant \\
\hline $5 a$ & 24 & $\begin{array}{l}\text { Incompatible } \\
\text { combination } \\
\text { practice }\end{array}$ & $\begin{array}{l}\text { alcohol } \\
\text { neutral }\end{array}$ & $\begin{array}{l}\text { soda } \\
\text { pleasant }\end{array}$ & $4 a$ & 24 & $\begin{array}{l}\text { Incompatible } \\
\text { combination } \\
\text { practice }\end{array}$ & $\begin{array}{l}\text { alcohol } \\
\text { neutral }\end{array}$ & pleasant \\
\hline $5 b$ & 24 & $\begin{array}{l}\text { Incompatible } \\
\text { combination } \\
\text { test }\end{array}$ & $\begin{array}{l}\text { alcohol } \\
\text { neutral }\end{array}$ & $\begin{array}{l}\text { soda } \\
\text { pleasant }\end{array}$ & $4 b$ & 24 & $\begin{array}{l}\text { Incompatible } \\
\text { combination } \\
\text { test }\end{array}$ & $\begin{array}{l}\text { alcohol } \\
\text { neutral }\end{array}$ & pleasant \\
\hline
\end{tabular}

Note. Blocks are shown for the positive alcohol-soda IAT and the positive alcohol ST-IAT. In the positive soda STIAT, the alcohol category was replaced by the soda category. Blocks 2 to 5 were repeated in the negative IAT and ST-IATs with the attribute categories 'unpleasant' and 'neutral'. The order of the positive and negative task versions was balanced across participants. The response assignment of the target categories was counterbalanced and remained constant across all tasks. The response assignment of the attribute categories was also counterbalanced so that half the participants always received the compatible combination task before the incompatible combination task whereas the other half always received the incompatible combination task before the compatible combination task.

Explicit Measures of Alcohol and Soda Cognitions. Participants filled out two expectancy questionnaires, one relating to alcohol and the other to soda, which consisted of 6 positive expectancy items ( $\alpha=.93, \alpha=.92$, respectively), and 6 negative expectancy items ( $\alpha=.81, \alpha=.92$, respectively). Each item asked participants to indicate on a Visual Analogue Scale (VAS) how much they agreed $(0=$ completely disagree, $100=$ completely agree) with the following statement: "After drinking alcohol/soda, I feel . ..." This statement was completed with the same words as presented during the IAT and ST-IATs, including the labels.

Attitudes toward alcohol and soda were assessed with two attitude questionnaires which consisted of 5 positive ( $\alpha=.86, \alpha=.89$, respectively) and 5 negative items $(\alpha=$ $.91, \alpha=.91$, respectively). In both questionnaires, participants indicated on a $100 \mathrm{~mm}$ 
VAS whether they agreed $(0=$ completely disagree, $100=$ completely agree $)$ with the statement: "I think drinking alcohol/soda is . ..", which was completed with the following words good, fun, smart, positive, and pleasant for the positive items and the words bad, stupid, boring, negative, and unpleasant for the negative items.

\section{Procedure}

After signing the informed consent form, participants performed the positive and negative IAT, the positive and negative alcohol ST-IAT and the positive and negative soda ST-IAT. Half the participants performed the IATs before the alcohol and soda ST-IATs, while this task order was reversed for the other half. Further, the order of the alcohol ST-IATs and the soda ST-IATs was also counterbalanced. Next, participants received the alcohol and soda expectancy and attitude questionnaires in balanced order, followed by the questionnaires on alcohol consumption and alcohol-related problems.

\section{RESULTS}

\section{IAT and ST-IAT Data Reduction}

IAT effects and ST-IAT effects were calculated with the D600 scoring algorithm (Greenwald, Nosek, \& Banaji, 2003). Mean response latencies were calculated for the two combination tasks of the positive and negative IAT, the positive and negative alcohol STIAT, and the positive and negative soda ST-IAT. For the positive and negative IAT, the D600 measure was calculated as the difference between these means so that higher scores indicate faster performance when alcohol was paired with positive attributes or negative attributes, respectively, and soda with neutral attributes than when alcohol shared a response with neutral attributes and soda with positive attributes or negative attributes, respectively. With respect to the positive and negative alcohol ST-IAT and the positive and negative soda ST-IAT, higher D600 scores reflect faster performance when either of the target concepts was paired with positive attributes or negative attributes, respectively, and neutral attributes were assigned to the other response than for the reversed combination. Further, following the formula presented by Greenwald et al. (2003), practice trials were always included, error penalties $(600 \mathrm{~ms})$ were given, and results were standardized at the level of the participant.

\section{Reliability of the IAT and ST-IAT}

To determine the reliability of the IAT and ST-IAT versions, (ST-)IAT scores computed from the practice trials were correlated with (ST-)IAT scores computed from the test trials (following the procedure outlined by Greenwald et al., 2003). Measures of internal consistency were then calculated by applying the Spearman-Brown correction to these 
correlations (cf. Karpinski \& Steinman, 2006). A reliability analysis on the positive IAT showed a lower level of internal consistency (adjusted $r=.55$ ) compared to the negative IAT which revealed a reasonable level of internal consistency (adjusted $r=.63$ ). Reliability analyses on the alcohol ST-IATs demonstrated greater internal consistency for the positive alcohol ST-IAT (adjusted $r=.71$ ) than for the negative alcohol ST-IAT (adjusted $r=.47$ ). Finally, the internal consistency of the positive soda ST-IAT (adjusted $r=.64$ ) was higher than the internal consistency of the negative soda ST-IAT (adjusted $r=.55$ ).

\section{Implicit Measures of Alcohol and Soda Attitudes}

Preparatory analyses revealed no influential outliers on IAT or ST-IAT data. With respect to the positive and negative IAT, Univariate Analyses of Variance (ANOVA) ${ }^{20}$ were used to examine the effect of two counterbalancing factors: the order in which the two task versions were presented and the order in which participants performed the IATs and the alcohol and soda ST-IATs. Results showed an effect of order of the positive and negative IAT on negative IAT scores, $F(1,58)=6.29, p=.015$. Other main effects as well as interaction effects were not significant $(p>.025)$. Follow-up analyses showed that positive IAT effects were not significant, $+(61)=1.05, p=.298$, while negative IAT effects were significant both when participants received the negative IAT before the positive IAT, $t(61)=$ $7.40, p<.001$, and when the negative IAT was performed after the positive IAT, $t(61)=$ $3.34, p<.001$. Hence, these findings demonstrate that participants held implicit negative attitudes toward alcohol relative to soda but do not provide support for implicit positive attitudes toward alcohol relative to soda.

For the alcohol and soda ST-IATs, we first examined the effect of three counterbalancing factors using ANOVAs ${ }^{20}$ : the order of the IATs and ST-IATs, the order of the alcohol and soda ST-IATs and the order of the positive and negative task versions. Results demonstrated that the order in which participants performed the alcohol ST-IATs and the soda ST-IATs influenced scores on the positive alcohol ST-IAT, $F(1,54)=8.62, p$ $=.005$. None of the other main effects or interaction effects reached significance $(p>$ .025). Similar to results with the IAT, a significant effect emerged in the negative alcohol ST-IAT, $t(61)=4.25, p<.001$, suggesting that participants had implicit negative attitudes toward alcohol. In addition, the positive alcohol ST-IAT also yielded a significant effect, revealing that participants also held implicit positive attitudes toward alcohol, but only when the alcohol ST-IATs were performed before the soda ST-IATs, $t(61)=4.70, p<$ .001 , but not when the soda ST-IATs were performed first, $t(61)=.44, p=.664$. Neither the positive soda ST-IAT nor the negative soda ST-IAT yielded a significant effect, $t(61)=$ $1.49, p=.142$, and $t(61)=1.07, p=.287$, respectively.

\footnotetext{
${ }^{20}$ In order to control for type I errors due to performing multiple ANOVAs, $\alpha$ was set at .025 .
} 
Interestingly, the alcohol ST-IAT scores and soda ST-IAT scores can inform us about the contributions of single implicit attitudes toward alcohol and single implicit attitudes toward soda to IAT scores. As can be seen in Table 2, the positive IAT and the negative IAT were uncorrelated with both alcohol ST-IAT versions and with both soda ST-IAT versions, suggesting that the alcohol ST-IATs and the soda ST-IATs measure qualitatively different underlying constructs than the alcohol-soda IATs. Thus, these findings demonstrate that the alcohol ST-IAT provides additional information that is not reflected in alcohol-soda IAT scores.

Table 2

Correlations between the IAT, alcohol ST-IAT and the soda ST-IAT.

\begin{tabular}{|c|c|c|c|c|c|c|c|}
\hline \multirow{2}{*}{ Variable } & & \multicolumn{2}{|c|}{ IAT } & \multicolumn{2}{|c|}{ Alcohol ST-IAT } & \multicolumn{2}{|c|}{ Soda ST-IAT } \\
\hline & & Positive & Negative & Positive & Negative & Positive & Negative \\
\hline \multirow[t]{2}{*}{$\overline{\mathrm{IAT}}$} & Positive & - & & & & & \\
\hline & Negative & $.27^{*}$ & - & & & & \\
\hline \multirow[t]{2}{*}{ Alcohol ST-IAT } & Positive & .20 & -.11 & - & & & \\
\hline & Negative & .15 & .17 & .12 & - & & \\
\hline \multirow[t]{2}{*}{ Soda ST-IAT } & Positive & .03 & -.15 & -.03 & .11 & - & \\
\hline & Negative & -.04 & -.09 & .05 & -.17 & $.29^{*}$ & - \\
\hline
\end{tabular}

Note. Partial correlations are shown, controlled for order of positive and negative task versions and for order of alcohol ST-IAT versions and soda ST-IAT versions. ${ }^{*}=$ Correlation is significant at the 0.05 level (2-tailed).

\section{Explicit Measures of Alcohol and Soda Cognitions}

Mean scores were calculated separately for positive and negative expectancy items of the alcohol and soda expectancy questionnaires as well as for the positive and negative attitude items of the alcohol and soda attitude questionnaires. Results showed that participants expected positive effects more from drinking alcohol $(M=62.26, S D=$ 16.63) than from drinking soda $(M=48.18, S D=18.68),+(61)=4.67, p<.001$. In addition, participants also expected more negative effects from drinking alcohol $(M=$ $31.83, S D=21.40)$ than from drinking soda $(M=20.27, S D=15.94), t(61)=3.99, p$ $<.001$. Also, participants' attitudes toward alcohol $(M=47.72, S D=17.03)$ were less positive compared to attitudes toward soda $(M=55.35, S D=21.55), t(61)=-2.26, p$ $=.027$, and alcohol attitudes $(M=38.03, S D=22.30)$ were also more negative compared to soda attitudes $(M=26.72, S D=22.61), t(61)=2.88, p=.005$. None of the partial correlations, controlled for order of positive and negative task versions and for order of the alcohol ST-IATs and the soda ST-IATs, between implicit measures of alcohol and soda attitudes with explicit measures of alcohol-related and soda-related cognitions were significant after Bonferroni correction ( $p>.01)$. 


\section{Prediction of Alcohol-Related Behavior}

An estimate of alcohol consumption was computed as the log-transformed average weekly alcohol consumption calculated from the alcohol use questionnaire. An estimate of alcohol-related problems was computed as the mean of the z-transformed RAPI and AUDIT log-transformed sum scores. The alcohol use estimate and the alcohol-related problems estimate were significantly correlated, $r=.68, p<.001$, indicating that participants with increased levels of alcohol consumption also experienced more alcoholrelated problems. As can be seen in Table 3, positive IAT scores as well as positive alcohol ST-IAT scores were related to alcohol use, demonstrating that stronger implicit positive attitudes toward alcohol relative to soda as well as single implicit positive alcohol attitudes are associated with higher levels of alcohol consumption. In addition, positive IAT scores were also significantly correlated with alcohol-related problems. Negative IAT scores and negative alcohol ST-IAT scores, on the other hand, were unrelated to alcohol use and alcohol-related problems.

Table 3

Correlations of implicit measures of alcohol and soda attitudes with alcohol-related behavior.

\begin{tabular}{|c|c|c|c|c|c|c|}
\hline \multirow{2}{*}{ Variable } & \multicolumn{2}{|c|}{ IAT } & \multicolumn{2}{|c|}{ Alcohol ST-IAT } & \multicolumn{2}{|c|}{ Soda ST-IAT } \\
\hline & Positive & Negative & Positive & Negative & Positive & Negative \\
\hline Alcohol use & $.39 * *$ & .03 & $.35^{* *}$ & -.09 & .06 & .08 \\
\hline Alcohol-related problems & $.43^{* *}$ & .02 & .22 & -.02 & .02 & -.09 \\
\hline
\end{tabular}

Note. Partial correlations are shown, controlled for order of positive and negative task versions and for order of alcohol ST-IAT versions and soda ST-IAT versions. ${ }^{* *}=$ Correlation is significant at the 0.01 level (2-tailed), ${ }^{*}=$ Correlation is significant at the 0.05 level (2-tailed).

In addition, the predictive validity of both the positive IAT and the positive alcohol ST-IAT was examined using hierarchical regression analysis. The regression analysis for the prediction of alcohol use is shown in Table 4. In step 1, none of the explicit alcoholrelated expectancies and attitudes significantly predicted alcohol use ${ }^{21}$. Importantly, entering the positive IAT and the positive alcohol ST-IAT in step 2 significantly increased the explained variance. Both the positive IAT and the positive alcohol ST-IAT significantly

\footnotetext{
${ }^{21}$ Given the high correlations between explicit alcohol-related expectancies and alcohol attitudes, alcohol-related expectancies and attitudes were also entered separately in hierarchical regression analyses for the prediction of alcohol use and alcohol-related problems. Results showed that alcohol use was significantly predicted by positive expectancies, $\beta=.38, p=.005$, but not by negative expectancies $(p>.40)$. Further, neither positive alcohol attitudes nor negative alcohol attitudes significantly predicted alcohol use $(p>.20)$. With respect to alcohol-related problems, results showed that both positive and negative alcohol expectancies were significant predictors, $\beta=.53$, $p<.001$, and $\beta=.26, p=.037$, respectively. Also, positive alcohol attitudes significantly predicted alcoholrelated problems, $\beta=.51, p=.008$, while negative alcohol attitudes did not $(p>.70)$.
} 
predicted alcohol use, demonstrating that the positive IAT and the positive alcohol ST-IAT predicted unique variance in alcohol consumption. Moreover, a series of regression analysis showed that the positive IAT predicted 16\% of the variance in alcohol use $19 \%$ above the variance explained by explicit measures and $7 \%$ beyond the variance explained by explicit measures and the positive alcohol ST-IAT) while the positive alcohol ST-IAT predicted $12 \%$ of the variance in alcohol use $17 \%$ beyond the variance explained by explicit measures and 5\% above the variance explained by explicit measures and the positive (AT).

Table 4

Summary of hierarchical regression analysis for the prediction of alcohol use by explicit alcohol-related cognitions and both the positive IAT and the positive alcohol ST-IAT.

\begin{tabular}{|c|c|c|c|c|c|c|c|}
\hline Step & Variable & B & SE B & $\beta$ & SE $\beta$ & $t$ & $p$ \\
\hline \multirow[t]{4}{*}{1} & Positive alcohol expectancies & .01 & .01 & .25 & .18 & 1.38 & .175 \\
\hline & Negative alcohol expectancies & .01 & .01 & .19 & .14 & 1.32 & .192 \\
\hline & Positive alcohol attitudes & -.00 & .01 & -.05 & .23 & -.21 & .832 \\
\hline & Negative alcohol attitudes & -.01 & .01 & -.31 & .22 & -1.44 & .156 \\
\hline \multirow[t]{6}{*}{2} & Positive alcohol expectancies & .01 & .01 & .24 & .17 & 1.41 & .163 \\
\hline & Negative alcohol expectancies & .00 & .01 & .06 & .14 & .45 & .653 \\
\hline & Positive alcohol attitudes & -.00 & .01 & -.05 & .214 & -.21 & .833 \\
\hline & Negative alcohol attitudes & -.01 & .01 & -.14 & .21 & -.68 & .499 \\
\hline & Positive IAT & .59 & .25 & .28 & .12 & 2.35 & .022 \\
\hline & Positive alcohol ST-IAT & .49 & .25 & .25 & .13 & 2.02 & .049 \\
\hline
\end{tabular}

The hierarchical regression analysis for the prediction of alcohol-related problems is shown in Table 5. In step 1, negative alcohol-related expectancies significantly predicted alcoholrelated problems while positive alcohol-related expectancies predicted alcohol-related problems at borderline significance ${ }^{21}$. Further, in step 2, the positive IAT significantly predicted alcohol-related problems whereas the positive alcohol ST-IAT was not predictive of alcohol-related problems. 
Table 5

Summary of hierarchical regression analysis for the prediction of alcohol-related problems by explicit alcohol-related cognitions and both the positive IAT and the positive alcohol ST-IAT.

\begin{tabular}{llcccccc}
\hline Step & Variable & $B$ & SE B & $\beta$ & SE $\beta$ & $t$ & $p$ \\
\hline 1 & Positive alcohol expectancies & .02 & .01 & .31 & .17 & 1.85 & .069 \\
& Negative alcohol expectancies & .01 & .01 & .27 & .13 & 2.00 & .050 \\
& Positive alcohol attitudes & .01 & .01 & .25 & .21 & 1.20 & 237 \\
& Negative alcohol attitudes & -.00 & .01 & -.07 & .20 & -.35 & .725 \\
$\quad$ Positive alcohol expectancies & .01 & .01 & .25 & .16 & 1.59 & .117 \\
$\quad$ Negative alcohol expectancies & .01 & .01 & .19 & .13 & 1.48 & .145 \\
$\quad$ Positive alcohol attitudes & .02 & .01 & .31 & .20 & 1.53 & .131 \\
$\quad$ Negative alcohol attitudes & .00 & .01 & .05 & .19 & .27 & .791 \\
$\quad$ Positive IAT & .65 & .21 & .34 & .11 & 3.09 & .003 \\
Positive alcohol ST-IAT & .09 & .21 & .05 & .12 & .41 & .682 \\
\hline
\end{tabular}

Note. $F(4,57)=5.85, p=.001, R^{2}=.29$ for step $1 ; F_{\text {change }}(2,55)=5.16, p=.009, R_{\text {change }}^{2}=.11$ for step 2 . Final model (step 2): $R^{2}=.40, R_{\text {odjusted }}^{2}=.34, F(6,55)=6.19, p<.001$.

\section{DISCUSSION}

The goal of the present study was to examine whether single implicit alcohol attitudes assessed with the ST-IAT are qualitatively different from relative implicit alcohol attitudes assessed relative to soda with the IAT. In addition, we also assessed single implicit attitudes toward soda with the ST-IAT in order to examine the contribution of both single implicit alcohol attitudes and single implicit alcohol attitudes to alcohol-soda IAT scores. Moreover, the alcohol-soda IAT as well as the alcohol ST-IAT and the soda ST-IAT were presented in a unipolar format so that implicit positive attitudes and implicit negative attitudes could be independently assessed. The present findings demonstrate that the alcohol ST-IAT indeed provides additional information compared to the alcohol-soda IAT. While the negative IAT showed an effect, indicating implicit negative attitudes toward alcohol relative to soda, the positive IAT showed no significant IAT effect. In contrast, both the positive and negative alcohol ST-IAT yielded significant effects, demonstrating implicit positive alcohol attitudes as well as implicit negative alcohol attitudes. Moreover, alcohol ST-IAT scores and soda ST-IAT scores were uncorrelated with IAT scores, indicating that single implicit attitudes assessed with the ST-IATs were qualitatively different from the comparative implicit attitudes measured with the IATs. Importantly, this conclusion was also supported by the finding that the positive IAT and the positive alcohol ST-IAT uniquely 
predicted alcohol use above explicit measures of alcohol-related expectancies and attitudes.

In the present study, both the negative IAT and the negative alcohol ST-IAT yielded significant effects, indicating that implicit alcohol attitudes are negative both when assessed relative to soda and when assessed alone. However, the positive alcohol ST-IAT provided support for implicit positive attitudes toward alcohol whereas the positive IAT did not demonstrate implicit positive attitudes toward alcohol relative to soda. Hence, the alcohol ST-IAT yielded additional information that was not captured by the alcohol-soda IAT. However, it is interesting to note that the positive alcohol ST-IAT only provided support for implicit positive attitudes toward alcohol when it was presented before the soda ST-IAT. This finding is in line with the idea that contextual features can shape implicit attitudes (cf. Mitchell, Nosek, \& Banaji, 2003). Possibly, the soda ST-IAT created a frame of reference so that the alcohol ST-IAT, when it was subsequently performed no longer assessed single implicit attitudes toward alcohol. Hence, these findings imply that researchers interested in assessing implicit attitudes toward single target concepts using a non-relative measure such as the ST-IAT should pay close attention to task orders to ensure the non-relative nature of the task.

To further our understanding of alcohol-soda IAT effects, we also assessed single implicit attitudes toward soda using a soda ST-IAT. Results demonstrated that both single implicit alcohol attitudes and single implicit soda attitudes assessed with ST-IATs were unrelated to alcohol-soda IAT scores. Thus, the present results suggest that the ST-IAT and the IAT captured qualitatively different constructs. It is likely that contrasting alcohol with soda in the IAT changes how alcohol is automatically evaluated compared to when it is not contrasted with a second category. Consistent with this conclusion, De Houwer, Geldof, and De Bruycker (2005) recently stated that it is likely that replacing a category in the IAT alters the properties by which categories are grouped. Thus, omitting or including the soda contrast in the IAT could change which features of the alcohol concept become salient and, therefore, the extent to which alcohol is more or less easily paired with positive and negative stimuli. It is important to note that the present findings with the positive alcohol-soda IAT were inconsistent with findings reported by Houben and Wiers (2006c) who did find evidence for implicit positive attitudes toward alcohol relative to soda. However, participants in the present study on average consumed about 7 alcoholic drinks a week whereas participants in the study by Houben and Wiers (2006c) consumed twice as much. Hence, if implicit positive attitudes toward alcohol increase in strength with increased levels of alcohol consumption, as was demonstrated both in this study as well as by Houben and Wiers (2006c), it is highly likely that differences between the participant samples in mean alcohol consumption were responsible for the differences between the two studies in overall effects with the positive alcohol-soda IAT.

Importantly, the positive alcohol-soda IAT and the positive alcohol ST-IAT uniquely predicted alcohol use above explicit alcohol-related expectancies and attitudes, which 
further supports the conclusion that the alcohol ST-IAT and the alcohol-soda IAT captured unique constructs. Consistent with previous studies (e.g., Houben \& Wiers, 2006c; Jajodia \& Earleywine, 2003), results with both the positive IAT and the positive alcohol ST-IAT demonstrated that implicit positive attitudes toward alcohol predicted alcohol use above explicit alcohol-related cognitions, while implicit negative attitudes toward alcohol, whether assessed with the IAT or the alcohol ST-IAT, showed no relationship with drinking behavior. While it was suggested that previous studies might have been unable to demonstrate a relationship between negative alcohol-soda IAT scores and drinking behavior because these IAT scores might reflect implicit soda attitudes rather than implicit alcohol attitudes, the present results indicate that this was probably not the case. Hence, the present results demonstrate that both implicit positive attitudes toward alcohol relative to soda and implicit positive attitudes toward alcohol alone are important determinants of drinking behavior. Presumably, implicit negative attitudes toward alcohol reflect extrapersonal evaluative associations with drinking alcohol, possibly shaped by culturally shared knowledge, that are not part of one's personally endorsed implicit attitudes and which are unrelated to drinking behavior. Support for this claim was recently found by Houben and Wiers (2006b) who found support for stronger positive than negative implicit attitudes toward alcohol in a personalized IAT that was designed to reduce extrapersonal contamination (Han, Olson, \& Fazio, 2006; Olson \& Fazio, 2004).

Finally, it should be noted that several other indirect measures have been developed that can assess implicit attitudes toward single attitude concepts besides the ST-IAT, including the Extrinsic Affective Simon Task (EAST; De Houwer, 2003) and the Go/No-Go Association Task (GNAT; Nosek \& Banaji, 2001). However, both these measures are unsuited to test implicit positive and negative attitudes separately. While no research has yet attempted to present the GNAT in a unipolar format, the EAST has been modified for this purpose, however, at the expense of losing its non-relative nature with respect to the target categories (Wiers, Ganushchack, Van de Ende, Smulders, \& de Jong, 2003). Further, de Liver, van der Pligt, and Wigboldus (in press), recently demonstrated that implicit positive and negative evaluative associations with single attitude objects can be assessed using a sequential priming paradigm. In addition, Karpinski and Steinman (2006) have introduced the Single Category Implicit Association Test (SC-IAT) which is conceptually similar to the ST-IAT, differing only in minor procedural details. Karpinski and Steinman provided support for the reliability and validity of the SC-IAT as a measure of single implicit attitudes and demonstrated that the SC-IAT, like the ST-IAT in the present study, yields additional information that is not reflected in relative IAT scores.

In conclusion, the present study demonstrates that it is useful to use measures of implicit alcohol attitudes that do not only present evaluative attribute categories in a unipolar format, but that also assess single implicit attitudes toward alcohol in addition to measures that assesses implicit alcohol attitudes relative to soda, since both types of tasks offer a unique contribution to the prediction of drinking behavior. Clearly, as we are 


\section{CHAPTER 8}

beginning to grasp the potential complexity of implicit alcohol attitudes as determinants of alcohol use and abuse, we need to find effective tools that can adequately capture these implicit attitudes. Hence, with the present research, we hope to contribute to as well as stimulate the further development of indirect measurement tools of implicit alcohol-related cognitions. 


\section{APPENDIX}

\section{Target Stimuli}

Alcohol: beer, wine, whisky, drink, vodka

Soda: Fanta, Coca Cola, Sprite, sinas (lemonade), ice-tea

\section{Attribute Stimuli}

Pleasant: amusing, sociable, happy, enjoyable, agreeable Unpleasant: down, lonely, unhappy, miserable, sad

Neutral homogenous 1: standard, normal, general, common, objective Neutral homogenous 2: level, average, everyday, middle, universal Neutral heterogeneous 1: steep, compact, curved, regular, literally Neutral heterogeneous 2: brown, normal, digital, historically, general 


$$
\text { D) }
$$




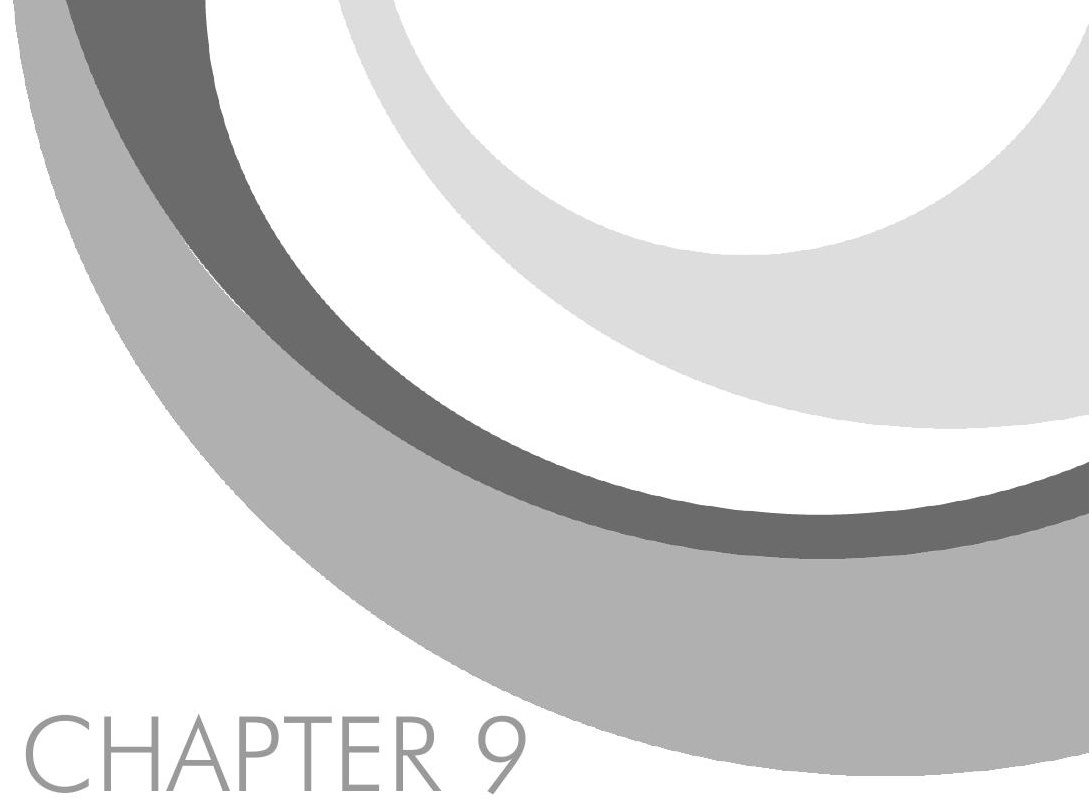

General Discussion 
The central focus of this thesis was to further our understanding of the automatic cognitive processes underlying alcohol use. This research interest was fueled by findings from previous IAT studies suggesting that implicit evaluative associations with alcohol are largely negative and not related to alcohol use (e.g., De Houwer, Crombez, Koster, \& De Beul, 2004; Wiers, van de Luitgaarden, van den Wildenberg, \& Smulders, 2005; Wiers, Van Woerden, Smulders, \& de Jong, 2002). However, recent research has demonstrated procedural limitations of the IAT that may compromise such an interpretation of previously found IAT effects. First, several lines of evidence suggest that IAT effects can be based on influences other than personal evaluative associations with the given target concepts (e.g., De Houwer, Geldof, \& De Bruycker, 2005; Mierke \& Klaver, 2001, 2003; Olson \& Fazio, 2004; Rothermund \& Wentura, 2001, 2004; Rothermund, Wentura, \& De Houwer, 2005). Second, the IAT assesses associations between two pairs of categories and can, thus, only indicate the relative strength of pairs of evaluative associations (that form a dichotomous attribute dimension in the IAT; e.g., positive versus negative) with one target concept (e.g., alcohol) relative to the other target concept (e.g., soft drinks). Therefore, the research presented in this thesis examined to what extent previously reported findings with the IAT might be due to such procedural limitations and focused on finding ways in which the IAT as a measure of automatic cognitive processes that determine drinking behavior might be improved.

\section{OVERVIEW AND DISCUSSION OF EMPIRICAL FINDINGS}

\section{Recoding Influences on Performance in the Alcohol-IAT}

In the first part of this thesis, it was examined to what extent IAT performance might be faster when alcohol shares a response with a negative attribute category (versus soda and a positive attribute category) than when alcohol is paired with a positive attribute category (versus soda and a negative attribute category) due to recoding processes rather than personal evaluative associations with the concept alcohol.

Controlling and Eliminating Recoding Influences in the Alcohol-IAT. In chapter 2, we tested whether the figure-ground asymmetry could account for results with the alcohol-IAT. More specifically, if alcohol-related stimuli are more salient compared to soda-related stimuli, the figure-ground account could explain this IAT effect since negative stimuli are generally more salient compared to positive stimuli and performance in the IAT should be faster when salient figure categories are consistently mapped onto the same response than when they are not assigned to the same response (Rothermund \& Wentura, $2001,2004)$. To test the influence of figure-ground asymmetries in the alcohol-IAT, figureground asymmetries between the target categories were reversed by manipulating the 
familiarity of the category exemplars (unfamiliar stimuli are generally more salient than familiar stimuli), to see if this would result in a reversal of the IAT effect. Although results confirmed that the manipulation succeeded in reversing figure-ground asymmetries between the alcohol and soda target categories, this reversal of figure-ground asymmetries only affected the size of the IAT effect but did not result in a reversal of the IAT effect. Further, effects in the familiar alcohol (vs. unfamiliar soda) IAT were negatively related to alcohol use, indicating weaker negative implicit associations with (familiar) alcohol (or stronger positive implicit alcohol associations) with increased levels of alcohol use. Importantly, controlling for figure-ground asymmetries did not attenuate this relationship between IAT effects and alcohol use. Thus, even though figure-ground asymmetries between the alcohol target category and the soda target category may contribute to effects in the alcohol-IAT, they cannot completely account for IAT effects or for their relationship with drinking behavior.

Figure-ground asymmetries, however, are just one possible source of recoding processes in the IAT. Therefore, in chapter 3, we used the IAT-RF, a variant of the IAT that eliminates all recoding influences by abandoning the block structure of the IAT (Rothermund, Teige-Mocigemba, \& Wentura, 2006), to study implicit alcohol-related associations. In contrast to the standard IAT procedure, compatible and incompatible response assignments are switched on a random trial-by-trial basis instead of in two different blocks. The implication is that the IAT-RF consists of two times two types of trials: trials are either compatible or incompatible with respect to their response assignment and trials either involve a switch or a repetition of the response assignment compared to the preceding trial. Compared to previous results with the standard IAT procedure (e.g., Wiers et al., 2002), results with the IAT-RF revealed less support (response assignment repetition trials) or no support (response assignment switch trials) for implicit negative associations with alcohol, indicating that previous findings with the standard alcohol-IAT at least partly reflected recoding processes. Moreover, predictive validity was demonstrated since IAT-RF effects for repetition trials were negatively related to alcohol use and predicted alcohol use better than explicit alcohol-related expectancies. This finding is consistent with the findings reported in chapter 2: Increased levels of alcohol use are characterized by weaker negative evaluative associations (or stronger positive evaluative associations) with alcohol. Further, IAT effects for switch trials were positively related to alcohol-related problems and predicted alcohol-related problems over and above the variance explained by explicit alcohol-related expectancies. This finding is consistent with the idea that negative alcoholrelated cognitions increase with the experience of alcohol-related problems but do not influence behavior until a certain threshold is reached (Jones \& McMahon, 1996). It should be noted that these findings also suggest that IAT-RF repetition trials and IAT-RF switch trials measure different underlying cognitive processes and can both provide unique information. 
Eliminating Extrapersonal Contamination in the Alcohol-IAT. Another way in which categories in the IAT may be recoded in a way that is inconsistent with the intended nominal features of the categories is when participants conceptualize the evaluative categories as normatively positive or negative. When attribute categories are re-defined in terms of normative information, it is possible that IAT effects reflect extrapersonal associations or cultural associative knowledge rather than personal associations. It is important to note that this kind of 'recoding' cannot be eliminated by abandoning the block structure of the IAT (cf. chapter 3), since it involves a re-definition of the nominal features of the categories rather than using other features (e.g., salience) to discriminate between the stimuli.

In chapter 4, we examined whether alcohol is perhaps more easily paired with a negative category than with a positive category due to negative extrapersonal information with respect to drinking alcohol. Here, we used a personalized IAT that reduces extrapersonal contamination by eliminating all reference to normative information to measure implicit alcohol associations. Consistent with the idea that participants are faster to combine alcohol with a negative attribute category than with a positive attribute category due to negative extrapersonal associations, the personalized IAT yielded positive implicit associations with alcohol rather than negative associations as found in earlier studies with the standard IAT. Moreover, positive implicit alcohol associations predicted alcohol use over and above explicit alcohol-related cognitions, indicating stronger positive associations with alcohol with increased levels of alcohol consumption. Hence, these findings replicate the relationship between implicit alcohol associations and alcohol use that was found in the studies reported in chapter 2 and 3. The important difference, however, is that this study for the first time demonstrated positive implicit associations with alcohol rather than negative implicit associations. Thus, these findings support the conclusion that negative extrapersonal associative knowledge contributes to effects in the standard IAT, causing faster performance when alcohol and negative are mapped onto the same response (vs. soda and positive) than when alcohol shares a response with positive (vs. soda and negative), and may even decrease its predictive validity. Further, results also showed that positive associations with alcohol significantly predicted alcohol-related problems so that participants who held stronger positive associations with alcohol also experienced more alcohol-related problems. However, similar to what was found in chapter 3, this relationship was completely mediated by alcohol use. When controlling for alcohol use in the hierarchical regression analysis, positive implicit alcohol associations no longer significantly predicted alcohol use, $\beta=-.18, t=-1.09, p=.285$, while alcohol use was the sole significant predictor of alcohol-related problems, $\beta=.35, t=2.22, p=$ .033.

One could question to what extent an IAT that presents stimuli that are standard across participants can capture personal associations since stimuli will undoubtedly differ in the extent to which they are both known and liked by participants (cf. Fadardi, Cox, \& 
Klinger, 2006). Therefore, in chapter 5, we measured implicit alcohol associations with a personalized IAT that no longer presented standard stimuli but instead individualized stimuli for both the target and attribute categories that were selected by participants themselves. A direct comparison between the standard IAT and the individualized personalized IAT showed that both tasks were strongly correlated with each other and that both tasks yielded negative implicit associations with alcohol, although the IAT effect was significantly smaller in the individualized personalized IAT version. Moreover, both tasks did not only show strong overlap with each other, they were also both related to alcohol use. Hence, these findings do not unequivocally support the conclusion that the individualized personalized IAT is more sensitive to personal associations than the standard IAT. On the one hand, the reduced IAT effect in the individualized personalized IAT, compared to the standard IAT, could be due to reduced influence by extrapersonal contamination. However, in contrast to what was found in chapter 4, there was no support for positive implicit alcohol associations, suggesting that extrapersonal contamination was reduced to a lesser extent in this study, although one could also argue that the disparity in findings was due to differences in the level of alcohol consumption of the participants in the two studies. On the other hand, the standard IAT and the individualized personalized IAT were similarly related to alcohol use, which seems to contradict the conclusion that the individualized personalized IAT succeeded in eliminating extrapersonal contamination (or would suggest that there was no extrapersonal contamination in the standard IAT in first place).

It should, however, be noted that in the study described in chapter 5, participants performed both the standard IAT and the personalized IAT in counterbalanced order, whereas in the study reported in chapter 4, participants only performed a personalized IAT. Possibly, performing the standard IAT after the individualized personalized IAT promoted a re-definition of the attribute categories in the standard IAT so that the evaluative categories were not conceptualized as normatively positive and negative. Similarly, performing the personalized IAT after the standard IAT might have caused a spill-over of extrapersonal contamination from the first IAT version to the latter IAT version. Importantly, such carryover effects might explain why the standard IAT and the individualized personalized IAT showed very similar patterns of results. Analyzing results separately for the two task orders provided some support for this conclusion. When participants performed the standard IAT first, the negative-alcohol IAT effect was significant in both the standard IAT, $t(23)=6.22$, $p<.001$, and in the individualized personalized IAT, $t(23)=3.95, p=.001$. However, when the individualized personalized IAT was performed first, the negative-alcohol IAT effect was significant in the standard IAT, $t(21)=3.38, p=.003$, but not in the individualized personalized IAT, $t(21)=.85, p=.403$. Moreover, standard IAT effects were significantly larger when the standard IAT was performed first compared to when the standard IAT was performed after the individualized personalized IAT, $t(44)=2.39, p=$ .021 . Similarly, effects with the individualized personalized IAT were borderline significantly 
smaller when the individualized personalized IAT was performed before the standard IAT than when it was performed after the standard IAT, $t(44)=-2.01, p=.051$.

Controlling or Eliminating Recoding Processes Increases the Validity of the Alcohol-IAT. Together, the studies described in the first part of this thesis demonstrate that recoding processes, in terms of performing the classification task based on other features than those initially intended, can influence IAT effects and thereby decrease the validity of the task as a measure of implicit alcohol-related associations. These findings further show that preventing recoding processes from affecting IAT performance increases the predictive validity of the IAT with respect to drinking behavior. Whereas previous studies have found no support to only moderate support for a relationship between evaluative implicit associations with alcohol and alcohol consumption (e.g., De Houwer et al., 2004; Wiers et al., 2002, 2005), the studies described in chapter 2 tot 5 all reveal a strong relationship between the IAT (variants) and behavior.

\section{Bipolar Target and Attribute Dimensions in the Alcohol-IAT: Double Jeopardy}

The IAT presents attribute categories in a bipolar format, which involves classifying attribute stimuli into two categories that typically form two poles of an evaluative dimension (e.g., positive vs. negative). Consequently, it is theoretically possible that a participant who has strong negative implicit alcohol associations as well as somewhat weaker positive implicit alcohol associations shows the same IAT score as a participant who has only negative implicit associations with alcohol. The implication is that this bipolar nature of the IAT may decrease the predictive validity of the IAT since positive implicit alcohol associations and negative implicit alcohol associations may be differentially related to alcohol use. In the second part of this thesis, unipolar variants of the IAT (e.g., positive vs. neutral) were used that allowed us to separately assess both positive implicit alcohol associations and negative implicit alcohol associations and their relationship to drinking behavior. Furthermore, the IAT also presents target categories in a bipolar format (e.g., alcohol vs. soda) and thus assesses implicit associations with one target concept relative to the other. Hence, it is unclear to what extent alcohol-IAT effects reflect implicit associations with the concept alcohol or with the contrast category soda. This issue was also examined in the second part of this thesis, where we assessed implicit alcohol associations relative to different contrast categories or with a non-relative variant of the IAT.

Unipolar Implicit Alcohol Associations. In chapter 6, evaluative implicit alcohol associations as well as implicit alcohol associations with arousal and sedation (cf. Wiers et al., 2002) were assessed using unipolar IATs. Results demonstrated both positive and negative implicit associations with alcohol as well as both arousal and sedation 
associations with alcohol. Importantly, implicit alcohol associations with arousal predicted alcohol use above explicit alcohol-related cognitions, which is consistent with findings with the bipolar IAT by Wiers et al. (2002). Further, implicit alcohol associations with sedation predicted alcohol-related problems above explicit alcohol-related cognitions, which could be related to research which has demonstrated that negative reinforcement motives are predictive of alcohol-related problems (e.g., Cooper, Frone, Russell, \& Mudar, 1995). Further, we demonstrated that positive implicit alcohol associations, in addition to implicit arousal associations, also borderline significantly predicted alcohol use over and above explicit alcohol-related cognitions. Negative implicit alcohol associations, in contrast, were unrelated to drinking behavior. In contrast, Wiers et al. (2002) found that implicit arousal associations with alcohol were related to level of alcohol use while implicit evaluative associations with alcohol were unrelated to alcohol use, and they linked their results to the incentive-sensitization theory which states that addictive substances sensitize the "wanting" system which is responsible for motivating behavior, but not the "liking" system which mediates the hedonic effects of addictive substances. Whereas findings with the unipolar IAT reported in chapter 6, suggest a tighter link between alcohol-related arousal and hedonic valence, it should be noted that such a pattern of results is consistent with normal appetitive behavior. Moreover, these results also fit nicely to the biphasic motivational model of emotion which states that emotion is organized around two motivational systems, one appetitive and one defensive. The motivational model further states that hedonic valence indicates which of these two motivational systems is active (i.e., pleasant = appetitive motivation and unpleasant = defensive motivation) while the degree of arousal reflects the degree of motivational activation (Bradley, 2000). Importantly, this model is not inconsistent with the incentive-sensitization theory of addictive behaviors, the main difference is that the incentive-sensitization theory predicts a dissociation between "wanting" (i.e., sensitized appetitive motivation) and "liking" (i.e., hedonic valence) when appetitive behavior becomes an addiction. In sum, results with unipolar IAT variants, thus, suggest that representations of appetitive reactions to alcohol cues (e.g., pleasure, activation) are an integral part of the associative cluster representing the concept alcohol with strong associative links to behavior. In contrast, negative hedonic valence or representations of the negative effects of drinking are probably not fully integrated in this associative cluster, perhaps because they are formed by reflective processes rather than automatic associative learning (cf. Deutsch \& Strack, 2006).

Further, the target concept of interest in the unipolar IATs that were used in chapter 6 was either alcohol or beer. Because unipolar beer IATs might tap more personal implicit associations whereas unipolar alcohol IATs might be more prone to extrapersonal contamination, it was expected that implicit associations with alcohol would be more negative and less positive compared to implicit associations with beer. Moreover, these two target concepts were contrasted either with the concept soda as in previous alcoholIAT studies or with the concept animals. Results demonstrated that the composition of the 
target dimension only significantly influenced results with the unipolar IAT that assessed negative implicit associations with the target categories. Specifically, implicit associations with beer were less negative when beer was contrasted with animals than when beer was contrasted with soda and implicit associations with beer versus animals were less negative than implicit alcohol associations regardless of the category that alcohol was contrasted with. Hence, the choice of the contrast category mainly affected results for the negative unipolar IAT, which may suggest that especially effects in this dimension may be susceptible to artificial influences. Finally, a second study indicated that a recoding account in terms of figure-ground asymmetries could not explain results with the unipolar IATs.

Non-Relative Implicit Alcohol Associations in a Unipolar Format. Although the results reported in chapter 6 indicated that the choice of the contrast category only affected results with the negative unipolar IAT but not results with the other unipolar IATs which were found to have predictive validity with respect to drinking behavior, it is important to note that the relativity of the IAT scores may still have deflated the validity of the unipolar IATs that were used. To further address this issue, positive and negative implicit alcohol associations were assessed in chapter 7 using a variant of the IAT, the Single-Target IAT (ST-IAT; Wigboldus, Holland, \& van Knippenberg, 2006; see also Karpinski \& Steinman, 2006), which presents only one target category and can, thus, measure implicit associations with single concepts. Moreover, the ST-IAT was used in a unipolar format similar to what was done with the IAT. Further, as in chapter 6, implicit associations were assessed with either alcohol or beer. Consistent with results described in chapter 6, there was no difference in the size of ST-IAT effects between the alcohol and the beer condition. Importantly, however, in this study, correlations with drinking behavior were examined separately per target condition (i.e., alcohol ST-IAT or beer ST-IAT) and these results did suggest that the alcohol ST-IAT version may be more influenced by extrapersonal associations than the beer ST-IAT. More specifically, consistent with results described in chapter 6, positive implicit associations with beer, but not negative implicit beer associations, were positively related to alcohol use. In contrast, neither the positive alcohol ST-IAT nor the negative alcohol ST-IAT was related to alcohol use. Hence, these results replicate the results described in chapter 6, demonstrating that positive implicit alcohol-related associations are involved in alcohol use while negative implicit alcoholrelated associations do not appear to play a role in drinking behavior.

In addition, these findings suggest that only the beer ST-IAT measured personal associations that are relevant to behavior while the alcohol ST-IAT may have been more sensitive to extrapersonal contamination since these ST-IAT scores were unrelated to behavior. It is important to note that the to-be-explained behavior was alcohol use and participants in this study indicated that, when they drank alcohol, they primarily consumed beer. Therefore, these findings are consistent with the findings reported in chapter 2 which 
indicated that an IAT that assessed implicit associations with an alcohol category consisting of alcoholic drinks that were familiar to the participants predicted behavior but not an IAT that measured implicit associations with an alcohol category that consisted of unfamiliar alcoholic drinks. Moreover, in light of these findings, correlations between the unipolar IATs and alcohol use as described in chapter 6 were re-analyzed per target condition. In this study as well, results indicated that the positive unipolar beer IAT versions (i.e., beer vs. soda and beer vs. animals) were related to alcohol use, $r=.33, p=.029$, but not the positive unipolar alcohol IAT versions (i.e., alcohol vs. soda and alcohol vs. animals), $r=$ $.06, p=.692$. The negative unipolar IAT was unrelated to drinking behavior in both conditions $(p>$.25). Moreover, the unipolar arousal IAT was related to alcohol use both in the alcohol condition, $r=.30, p=.046$, and in the beer condition, $r=.37, p=.012$, while the unipolar sedation IAT was related to drinking behavior only in the beer condition, $r=.38, p=.009$, but not in the alcohol condition, $r=-.11, p=.471$. Hence, these findings indicate that implicit arousal associations were related to alcohol use regardless of whether they were assessed specifically with respect to beer or more generally with respect to alcohol, whereas implicit positive associations and implicit sedation associations only related to alcohol consumption when they were specific to beer. Further, inspection of participants' alcohol consumption showed that these participants on average drank 15.07 $(S D=10.55)$ alcoholic drinks per week including $11.82(S D=11.75)$ glasses of beer. Together, these findings, thus, underscore the importance of choosing the appropriate target concept given the behavior that needs to be explained. More specifically, the closer the overlap between the processes that determine the IAT score and those that determine the actual behavior that one wants to predict, the better the IAT score will be able to predict the behavior (see also De Houwer, 2006).

Finally, in chapter 8, unipolar ST-IATs that assessed single implicit associations with alcohol and single implicit associations with soda were compared with unipolar IATs that measured implicit associations with alcohol relative to soda. Findings indicated that the unipolar alcohol and soda ST-IATs and the unipolar alcohol-soda IATs captured qualitatively different underlying constructs. Hence, these results suggest that the alcohol target concept is conceptualized differently depending on whether it is presented relative to another target category or in a non-relative way. Importantly, both the positive unipolar alcohol ST-IAT and the positive unipolar alcohol-soda IAT predicted alcohol use. These findings are, thus, consistent with findings reported in chapter 6 and 7, demonstrating that stronger positive implicit associations predict an increase in alcohol consumption. Further, Thush and Wiers (in press) also found similar results with the positive unipolar ST-IAT in adolescents, and, in addition, demonstrated that both the unipolar positive ST-IAT and a unipolar arousal ST-IAT predicted drinking behavior after one year which is consistent with findings with unipolar IATs reported in chapter 6. Further, the positive unipolar alcoholsoda IAT, but not the positive unipolar alcohol ST-IAT, predicted alcohol-related problems and, unlike previous results (chapter 3 and 4), this relationship was not mediated by 
alcohol use. However, it should be noted that participants in this study, in contrast to participants in the studies described in chapter 3 and 4, were light drinkers who reported very little alcohol-related problems. Finally, consistent with other findings with unipolar IAT variants reported in this thesis, the negative unipolar alcohol-soda IAT and the negative unipolar alcohol ST-IAT were unrelated to drinking behavior. In sum, these findings indicate that the relative nature of the IAT does not necessarily deflate its predictive validity but rather that the relative IAT and the non-relative ST-IAT both have unique predictive validity.

\section{Main Conclusions}

1. In addition to implicit associations with alcohol, IAT effects reflect recoding processes that deflate both the construct validity and the predictive validity of the task (chapter 2 to 5).

2. Eliminating or controlling for recoding processes in the IAT reveals that implicit alcohol associations are meaningfully related to drinking behavior (chapter 2 to 5).

3. Using IATs that present the attribute dimension in a unipolar format shows that implicit positive associations with alcohol are a driving force behind alcohol use while negative implicit alcohol associations do not appear to influence drinking behavior (chapter 6 to 8).

4. Implicit associations with alcohol relative to soda and single implicit alcohol associations provide unique information with respect to drinking behavior (chapter 8).

5. Since the overlap between the cognitive processes that determine IAT performance and the cognitive processes underlying the to-be-explained behavior determines the predictive validity of the IAT, one should select appropriate target concepts given the behavior that needs to be explained rather than general target concepts (chapter 2 and chapter 7).

\section{METHODOLOGICAL ISSUES}

\section{Psychometric Properties}

A necessary prerequisite for any measure of individual differences is that it should show adequate reliability. To examine reliability of all IAT variants that were tested in this thesis, internal consistencies were calculated by correlating the IAT effect measure calculated for the practice trials (i.e., IAT effect based on the first half of the compatible combination trials and the first half of the incompatible combination trials) with the IAT effect measure calculated for the test trials (i.e., IAT based on the second half of the compatible and 
incompatible combination trials) (as in Greenwald, Nosek, \& Banaji, 2003). However, dividing a measure into halves underestimates the reliability of the entire measure, and therefore we also calculated correlations adjusted with the Spearman-Brown correction which compensates for this underestimate of the true internal consistency (cf. Karpinski \& Steinman, 2006). Internal consistencies for all IAT variants used in this thesis are presented in Table 1.

Table 1

Internal consistencies of the IAT variants used in this thesis.

\begin{tabular}{|c|c|c|c|}
\hline IAT Type & IAT Variant & r & adjusted $r$ \\
\hline \multirow[t]{6}{*}{ Bipolar positive vs. negative } & Familiar alcohol vs. unfamiliar soda IAT (chapter 2) & .84 & .92 \\
\hline & Unfamiliar alcohol vs. familiar soda IAT (chapter 2) & .87 & .93 \\
\hline & IAT-RF & .35 & .51 \\
\hline & Personalized IAT (chapter 4) & .61 & .76 \\
\hline & Personalized individualized IAT (chapter 5) & .67 & .80 \\
\hline & Standard IAT (chapter 5) & .70 & .83 \\
\hline \multirow[t]{6}{*}{ Unipolar positive vs. neutral } & alcohol/beer vs. soda/animals IAT (chapter 6) & .46 & .63 \\
\hline & Alcohol ST-IAT (chapter 7) & .63 & .77 \\
\hline & Beer ST-IAT (chapter 7) & .16 & .29 \\
\hline & Alcohol vs. soda IAT (chapter 8) & .38 & .55 \\
\hline & Alcohol ST-IAT (chapter 8) & .56 & .71 \\
\hline & Soda ST-IAT (chapter 8) & .50 & .64 \\
\hline \multirow[t]{6}{*}{ Unipolar negative vs. neutral } & alcohol/beer vs. soda/animals IAT (chapter 6) & .44 & .61 \\
\hline & Alcohol ST-IAT (chapter 7) & .17 & .29 \\
\hline & Beer ST-IAT (chapter 7) & .29 & .45 \\
\hline & Alcohol vs. soda IAT (chapter 8) & .46 & .63 \\
\hline & Alcohol ST-IAT (chapter 8) & .31 & .47 \\
\hline & Soda ST-IAT (chapter 8) & .38 & .55 \\
\hline Unipolar arousal vs. neutral & alcohol/beer vs. soda/animals IAT (chapter 6) & .52 & .68 \\
\hline Unipolar sedation vs. neutral & alcohol/beer vs. soda/animals IAT (chapter 6) & .46 & .63 \\
\hline
\end{tabular}

Note. Internal consistencies were calculated by correlating ( $r$ ) IAT effects based on practice trials (i.e., first half of the IAT combination trials) with IAT effects based on test trials (i.e., second half of the IAT combination trials), and applying the Spearman-Brown correction (adjusted $r$ ). 
Results demonstrated good internal consistencies for all bipolar IAT variants used in chapter 2 to 5, which were similar to internal consistencies that have been reported in other IAT studies (e.g., Bosson, Swann, \& Pennebaker, 2000). In contrast, internal consistencies of the unipolar IATs were much lower which is probably due to the use of the neutral attribute categories in these IAT variants. More specifically, the neutral categories that were used in order to create a unipolar format for the attribute dimension typically consisted of a set of assorted stimuli which were all evaluated as neutral but did not form a category that was as coherent as for example the positive attribute category. It should be noted that in the study reported in chapter 8 , unipolar formats were created for the IAT variants using either neutral attribute categories consisting of diverse neutral words (similar to those used in the studies described in chapter 6 and 7) or using neutral categories that consisted of neutral words that were all synonyms of the word 'neutral'. It was expected that the latter neutral categories would be more coherent compared to the diverse neutral categories. However, results yielded similar results for both types of unipolar formats, including internal consistencies.

Future research should further examine how the reliability of unipolar IATs can be improved given that these IAT variants can provide important additional information compared to standard bipolar IATs. Further, a possibly valuable alternative to using unipolar IATs is to use the Single Attribute IAT (SA-IAT; Penke, Eichstaedt, \& Asendorpf, 2006) which presents only one attribute category but is further comparable to the standard IAT. The SA-IAT is, thus highly similar to the ST-IAT, with the difference that in the SA-IAT, the attribute dimension is asymmetric whereas in the ST-IAT, asymmetry concerns the target dimension. Importantly, satisfactory reliability was demonstrated for the SA-IAT, indicating that it can provide a reliable measure of unipolar associations (Penke et al., 2006).

\section{Construct Validity}

It should be noted that positive and negative attribute concepts were operationalized in two different ways in this thesis. Specifically, some studies reported in this thesis used IAT variants that assessed associations between the target concepts and positive and negative attribute categories that consisted of alcohol-related positive and negative expectancies (chapter 2, 3, and 8) similar to what was done in the study by Wiers et al. $(2002,2005)$ and in the study by De Houwer et al. (2004). Other studies used IAT variants that measured associations of the target concepts with positive and negative attribute categories that were made up of general positive and negative stimuli (chapter 5, 6, and 7), which is similar to social cognition research with the IAT (e.g., Greenwald, McGhee, \& Schwarz, 1998). For simplicity, IAT variants that used alcohol-related expectancies to operationalize the attribute categories will be referred to as expectancy IAT variants while IAT variants that used general positive and negative stimuli to operationalize the attribute categories will be referred to as attitude IAT variants. 
While it has been demonstrated that differences in the features of the attribute categories can influence both IAT effects (e.g., Bluemke \& Friese, 2006) and their relationship to self-report measures (e.g., Hofmann, Gawronski, Gschwendner, Le, \& Schmitt, 2006), influences of attribute stimuli on IAT effects have been mainly studied with respect to cross-category associations. More specifically, it has been demonstrated that using an attribute category that is conceptually related to one target category and another attribute category that is conceptually related to the other target category can give rise to stronger IAT effects. However, it is unlikely that cross-category associations differentially influenced the expectancy IAT variants and the attitude IAT variants since the two attribute categories were either not conceptually related to either of the target categories (attitude IATs) or both conceptually related to the alcohol target category but not to the soda target category (expectancy IATs). Still, findings are perhaps not directly comparable between studies in which positive and negative implicit associations were differently operationalized.

Let us first turn to the studies that are directly comparable. Wiers et al. $(2002,2005)$ as well as De Houwer et al. (2004) demonstrated that alcohol was more easily paired with a negative attribute category consisting of negative alcohol-related expectancies, than with a positive attribute category that consisted of positive alcohol-related expectancies. This result was replicated and extended in chapter 2, where we demonstrated the same IAT effect regardless of manipulations of figure-ground asymmetries. Further, it was demonstrated in chapter 3 that this effect was reduced and even eliminated when using a recoding-free variant of the IAT. However, even though positive and negative attribute categories in this recoding-free IAT variant consisted of alcohol-related expectancies, it should be noted that this IAT variant used different attribute category labels which may reduce the comparability of findings with this variant and results with other IAT variants used in this thesis. Further, findings with unipolar expectancy IAT variants indicated that alcohol is more easily paired with both a negative category consisting of alcohol-related negative expectancies and with a positive category consisting of positive alcohol-related expectancies than with a neutral category, but only when a non-relative IAT variant is used that assesses single implicit associations with alcohol (chapter 8). Importantly, whereas Wiers et al. (2002, 2005) and De Houwer et al. (2004) did not find a relationship between effects with expectancy IATs and level of alcohol use, findings with expectancy IAT variants reported in this thesis that increased levels of alcohol use are related to stronger positive implicit alcohol associations (chapter 2, 3, and 8).

Even though the attribute categories were differently operationalized in the attitude IAT variants, findings with attitude IAT variants were similar to those reported with expectancy IAT variants. First, bipolar attitude IATs also demonstrated that alcohol was more easily paired with a negative attribute category than with a positive attribute category (chapter 5). Moreover, this IAT effect was reduced (chapter 5) and even eliminated (chapter 4) when using IAT variants that eliminated extrapersonal contamination. Second, unipolar attitude IAT variants also yielded both positive and negative implicit alcohol 
associations (chapter 6 and 7). Similar to findings with expectancy IAT variants, results with attitude IAT variants also showed that implicit alcohol associations are stronger with increased alcohol use (chapter 4, 5, 6, and 7). Notwithstanding the fact that results are similar regardless of whether expectancy IAT variants were used or attitude IAT variants, one can, however, not rule out that these different IAT variants assessed different underlying constructs, and future research should, thus, further examine this issue.

\section{FUTURE DIRECTIONS}

\section{Do Implicit Alcohol Associations Determine Drinking Behavior: The Issue of Causality}

It is important to note that all studies reported in this thesis were cross-sectional in nature and exclusively relied on correlational data. Moreover, the dependent variable in all studies was drinking behavior of the week preceding the study. Hence, while results demonstrate that both explicit and implicit alcohol-related cognitions are (retrospectively) uniquely related to drinking behavior, the question whether these implicit and explicit alcohol-related cognitions are a cause or a consequence of drinking behavior cannot be answered. To further examine this issue of causality, future research should examine the relationship between implicit alcohol-related associations measured with the IAT and prospective drinking behavior, controlled for past drinking behavior. Similarly, Stacy (1997) demonstrated in a longitudinal study that both implicit alcohol-related memory associations measured with word association measures and explicit alcohol-related expectancies predicted prospective alcohol use beyond the variance explained by past alcohol use. Further, it is interesting to note that, in the study by Stacy (1997), implicit memory associations were stronger predictors of prospective alcohol use than explicit alcohol-related expectancies. Consistent with this finding, Jones, Corbin, and Fromme (2001) concluded that the variance in alcohol use explained by explicit alcohol-related expectancies decreases previous drinking behavior is included in the analyses. Together, these results suggest that implicit alcohol-related cognitive processes might be more important predictors of prospective alcohol use than explicit alcohol-related cognitions, and explicit alcohol-related expectancies in particular. Thus, it should be interesting to examine the relative contributions of implicit associations assessed with IAT and explicit alcohol-related expectancies and attitudes in the prediction of prospective drinking behavior. Moreover, as a more direct approach to examining the causality of the relationship between implicit alcohol-related associations measured with the IAT and alcohol use, future research could (temporarily) increase the strength of implicit positive associations with alcohol, for example through an evaluative conditioning procedure, to examine whether such a procedure produces parallel increases in alcohol consumption. 


\section{Executive Functioning as a Moderator of the Relationship between Implicit Alcohol-Related Cognitions and Drinking}

It was recently suggested that the dynamic interplay between automatic cognitive processes and controlled processes may be influenced by individual differences in executive control or executive functioning (e.g., Barrett, Tugade, \& Engle, 2004; Payne, 2005; Stacy, Ames, $\&$ Knowlton, 2004). Although there is no consensus definition of executive function, the term is generally used to refer to a range of top-down cognitive processes and abilities that enable flexible goal-directed behavior. Examples of such processes include planning and monitoring behavior, goal management, task switching, and overriding automatic responses (see also Baddeley, 1986; Norman \& Shallice, 1986). Hence, executive functioning could be an important moderator of whether automatic cognitive processes influence behavior or not. Indeed, recent research supports such a moderating role of executive functions. Payne (2005) for example measured executive control with the antisaccade task and estimated both automatic racial bias and executive control from performance on the weapon identification task and evaluative priming. Importantly, Payne (2005) demonstrated that executive control moderated the relationship between automatic racial bias and behavioral responses, such that automatic bias was more likely to be expressed in behavioral errors or in social judgments for participants with poor executive control than among participants with good executive control. Further, Grenard, Ames, Wiers, Thush, Sussman, and Stacy (2006) showed that substance-related associations, measured with the word association task, were stronger predictors of substance use among participants with lower working memory capacity than among participants with higher working memory capacity, as measured with the self-ordered pointing task. Finally, Thush, Wiers, Ames, Grenard, and Stacy (2006) demonstrated the same moderating influence of working memory capacity on the relationship between implicit alcohol associations with arousal, assessed with a unipolar IAT, and alcohol use.

Together, these results are promising and indicate that executive functions can moderate the influence of automatic processes on behavior. Therefore, future research should further examine whether executive functions act as a moderator of the relationship between implicit alcohol associations assessed with the IAT and drinking behavior. Further, there is still debate on whether executive functions are supported by a single unitary process (e.g., Barrett, Tugade, \& Engle, 2004) or a diverse array of cognitive processes (e.g., Miyake, Friedman, Emerson, Witzki, \& Howerter, 2000). Thus, it would be interesting to explore whether the relation between implicit alcohol associations measured with the IAT and behavior is moderated by general executive functioning or by specific executive functioning processes, such as, for instance, the ability to inhibit automatic responses. Finally, these new insights also indicate that it might be interesting to investigate whether executive functions can be trained and improved (see for example Evans, 2005, for training of executive functions in the context of executive dysfunction; Olesen, Westerberg, 
\& Klingberg, 2004, for training of working memory), and whether such an approach could be effective in changing the automatic influence of implicit alcohol associations on drinking behavior. Especially people with poorer executive control might benefit from training aimed at improving their ability to override the influence of their automatic cognitive processes on behavior.

\section{Changing Implicit Alcohol-Related Cognitions}

Findings advanced in this thesis suggest that evaluative implicit associations are an important determinant of drinking behavior. Therefore, a prerequisite for intervention strategies aimed at reducing drinking is that they should not only target explicit alcoholrelated cognitive processes but also implicit alcohol-related cognitive processes. Interestingly, Gawronski and Bodenhausen (2006) recently proposed the APE (AssociativePropositional Evaluation) model that specifies under which conditions changes are expected in implicit cognitions, explicit cognitions or both. More specifically, they advocate that changes in implicit or explicit cognitions depend on which of the two processes is influenced but also on whether change in one type of process mediates change in the other type of process. As such, it is advocated that a direct change in implicit cognitive processes can be expected either when the associative structure is changed, for example by introducing new associations through evaluative conditioning, or when the temporal activation of preexisting associations is changed, for example due to context cues. Moreover, changes in explicit cognitive processes can indirectly lead to the construction of new implicit associations or to the activation of implicit associations that were not activated before, thereby causing changes in implicit cognitive processes.

A direct approach to changing implicit cognitive processes, thus, is the construction of new implicit associations through evaluative conditioning. An example of this approach can be found in a series of studies by Olson and Fazio (2006), demonstrating that an evaluative conditioning procedure reduced prejudice against black people as indicated by an evaluative priming measure of implicit racial attitudes. Given these promising results, it should be interesting to explore whether an evaluative conditioning procedure could also succeed in changing implicit alcohol-related associations and whether such an approach will also produce corresponding changes in drinking behavior. A more indirect approach to changing implicit cognitive processes is to induce a change in explicit cognitive processes, which then leads to a corresponding change in implicit cognitive processes. An example of this approach is found in Wiers et al. (2005), who examined whether an expectancy challenge would be effective in changing both explicit and implicit alcoholrelated cognitions. While they found a decrease in explicit positive arousal alcohol-related expectancies, there was no evidence for a change in implicit alcohol-related cognitions assessed with the IAT. However, Gawronski and Bodenhausen (2006) argue that the crucial determinant of whether changes in explicit cognitive processes will produce 
changes in implicit cognitive processes is whether explicit changes lead to a mere negation of implicit cognitions that are already activated or to an affirmation of implicit cognitions that were not activated before. Importantly, only the latter case will provide the necessary conditions for producing a change in implicit cognitive processes. Hence, it could be argued that the expectancy challenge in the study by Wiers et al. (2005) mainly negated positive arousal expectancies with respect to drinking, which could explain why no support was found for a change in implicit alcohol-related cognitions. Further, based on these considerations, it should also be interesting to examine whether training participants in the affirmation of negative evaluations with respect to alcohol could be able to change both explicit alcohol-related cognitions as well as implicit alcohol-related cognitions.

Nevertheless, it should be noted that findings reported in this thesis indicate that negative evaluative associations with the concept alcohol typically already exist without influencing drinking behavior as has also been found for explicit negative expectancies (e.g., Wiers, Gunning, \& Sergeant, 1998; Wiers, Sergeant, \& Gunning, 2000). Perhaps negative implicit alcohol associations do not affect behavior because they are not activated in contexts where people typically drink alcohol. If this is the case, it is perhaps equally important to explore ways in which the temporal activation of negative implicit alcohol associations can be increased in contexts where people consume alcohol.

\section{CONCLUDING REMARKS}

In contrast to previous studies with the IAT, the findings in this thesis demonstrate that evaluative implicit associations with the concept alcohol are involved in drinking behavior, in such a way that stronger positive implicit alcohol associations are related to increased alcohol consumption. Further, in most studies, positive implicit associations with alcohol predicted drinking above the variance explained by explicit alcohol-related cognitions, supporting the value of supplementing self-report measures with implicit measures in alcohol research. The research in this thesis was also focused on developing and testing modifications of the IAT that overcome some of the limitations of the standard IAT procedure. Although this approach yielded new insights with respect to cognitive processes that may influence alcohol use relatively automatically, it is also evident that more research is needed that is aimed at developing indirect measurement procedures that can offer reliable and valid measures of automatic cognitive processes. Finally, given that implicit alcohol-related cognitions can be important determinants of alcohol use and misuse, future directions should include efforts aimed at changing automatic alcohol-related cognitive processes as well as their impact on drinking behavior. 



\section{Summary}

Almost everyone enjoys a few drinks from time to time, as do I. However, excessive alcohol use can cause both serious health problems as well as social problems. For this reason, researchers have been trying to identify the cognitive processes underlying alcohol abuse for many decades, so that appropriate interventions can be developed that are aimed at reducing alcohol intake. Until a few years ago, most research on the cognitive processes involved in alcohol use and abuse has been based on participants' self-reporting of the reasons for their alcohol use. However, the increased understanding of addictive behaviors from the perspective of dual-process models as well as the development of indirect measurement procedures have recently fostered an interest in the role of so-called implicit or automatic cognitive processes in alcohol abuse.

More specifically, dual process models state that behavior is determined by the dynamic interplay of two qualitatively different cognitive processes: fast, spontaneous, associative, automatic cognitive processes and slow, deliberative, rule-based, controlled cognitive processes (e.g., Deutsch \& Strack, 2006; Evans \& Coventry, 2006; Fazio \& TowlesSchwen, 1999). Whereas self-report measures can be easily used to tap controlled cognitive processes, it is far more difficult to measure automatic cognitive processes since participants may not always be able to report on the automatic cognitive processes underlying their behavior. Importantly, it has also been suggested that automatic cognitive processes become increasingly important as excessive alcohol use develops into an addiction (e.g., Bechara et al., 2006; Deutsch \& Strack, 2006; Evans \& Coventry, 2006), stressing the importance of developing appropriate measures that can be used to measure automatic cognitive processes.

With the development of indirect measures, researchers believed that they had found the appropriate tools for measuring automatic cognitive processes that steer behavior. Unlike direct self-report measures, indirect measures do not rely on self-report but instead they infer cognitive processes indirectly from performance on a speeded reaction-time task. Hence, indirect measures could be uniquely suited to tap relatively fast, automatic cognitive processes while at the same time limiting participants' ability for controlled responding. One of the indirect measures that received most attention over the last few years is the Implicit Association Test (IAT; Greenwald, McGhee, \& Schwartz, 1998).

The IAT is a double categorization task in which participants are instructed to simultaneously classify stimuli that are presented one by one on the computer screen into two target categories and two attribute categories with only two response keys. The two target categories typically represent the concepts in which the researcher is interested, such as 
alcohol versus soft drinks, whereas the two attribute categories often represent evaluative concepts such as positive versus negative. Further, in two different phases of the task, the target and attribute categories are paired in two different combinations. During one phase, participants for example have to classify both alcohol and positive stimuli with one response key and soft drinks and negative stimuli with the other response key. In the second phase, this response assignment is then reversed so that alcohol shares a response key with negative stimuli while soft drinks are assigned to the same response key as positive stimuli. The idea behind the task is that participants will respond faster when target categories and attribute categories are combined in a way that corresponds to participants' implicit associations between the target concepts and attribute concepts. For instance, if participants respond faster when alcohol and positive stimuli have to be classified with the same key and soft drinks and negative stimuli with the other, than when alcohol shares a key with negative stimuli and soft drinks are mapped onto the same key as positive stimuli, this would suggest that these participants have positive implicit associations with alcohol. As such, the IAT can be used to index how participants automatically evaluate certain target concepts.

This thesis focuses on the IAT as an indirect measure of automatic alcohol-related cognitive processes. The last few years, findings with the IAT repeatedly suggested negative implicit associations with alcohol, regardless of level of alcohol consumption, that were in clear contrast to participants' self-reported positivity with respect to drinking alcohol (e.g., Wiers et al., 2002, 2005). Further, research demonstrated that, while heavy and light drinkers could not be differentiated based on their implicit evaluative associations with alcohol, heavy but not light drinkers hold implicit associations between alcohol and arousal (e.g., Wiers et al., 2002). Hence, these findings suggest that implicit evaluative associations play little to no role in drinking behavior, which appears to be determined more by implicit arousal associations and explicit positive cognitions with respect to drinking alcohol. Nevertheless, recent research has also uncovered limitations of the IAT procedure that may complicate the interpretation of IAT effects. Therefore, the focus of this thesis was twofold. First, it was examined whether limitations in the IAT procedure might have been responsible for previous results with the IAT. Second, it was examined how the IAT might be improved so that it would be better suited to tap implicit alcohol-related cognitive processes.

In the first part of this thesis, it was examined to what extent findings with the alcoholIAT reflect recoding influences rather than implicit evaluative associations with the concept alcohol. Recoding refers to the phenomenon that target and attribute stimuli may be categorized on the basis of features other than those intended by the researcher. More specifically, in the case of the alcohol-IAT, participants may classify all stimuli based on, for example, salience or familiarity rather than categorizing target stimuli as being related to alcohol or soft drinks and attribute stimuli as being positive or negative. In chapter 2, it was demonstrated that manipulating recoding processes based on the salience of the categories in the IAT influenced the overall size of IAT effects but not their direction. Further, controlling for recoding based on salience did not attenuate the relationship between implicit evaluative 
associations with alcohol and drinking behavior: Stronger negative implicit associations with alcohol were related to a decrease in alcohol use.

Nevertheless, recoding based on salience is just one of the possible recoding influences that can influence IAT performance. Therefore, in the remaining chapters of the first part of this thesis, implicit evaluative associations with alcohol were measured using new variants of the IAT, which should eliminate or diminish the contribution of recoding processes to IAT effects. In line with the hypothesis that previous findings with the standard IAT at least to some extent may have reflected recoding processes, recoding free variants of the IAT yielded considerably less support for implicit negative associations with alcohol, and even revealed positive implicit associations with alcohol. Importantly, throughout the studies reported in the first part of this thesis, implicit evaluative associations with alcohol were demonstrated to be related to drinking behavior in such a way that stronger negative implicit associations with alcohol were related to reduced alcohol use and stronger positive implicit associations to increased alcohol intake. Thus, together these results firstly suggest that controlling for recoding processes in the IAT may increase the validity of the task as a measure of implicit associations and secondly, that implicit evaluative associations with alcohol do play a role in drinking behavior.

The second part of this thesis comprised studies that were concerned with the dual bipolarity of IAT categories. More specifically, participants typically have to categorize stimuli into two times two categories. Two categories, for example alcohol and soft drinks, represent the poles of the target dimension, and the other two categories, for example positive and negative valence, represent the poles of the attribute dimension. Hence, both the target dimension and the attribute dimension are bipolar. Due to the bipolarity of the target dimension, the IAT only informs us whether, for instance, alcohol is associated more strongly with negative than with positive when compared to soft drinks. Hence, implicit alcohol associations assessed with the IAT are always relative to a contrast category such as soft drinks. Therefore, the IAT may reflect both implicit associations with alcohol, implicit associations with soft drinks, or both, which complicates the interpretation of IAT effects. Further, due to the bipolarity of the attribute dimension it is impossible to assess whether positive and negative implicit associations with alcohol exist simultaneously, which is problematic given that ambivalence is typically quite strong in the case of alcohol use (Conner \& Sparks, 2002).

In order to separately assess both positive and negative implicit associations with alcohol as well as their relationship with behavior, studies reported in the second part of this thesis used variants of the IAT that presented the attribute dimension in a unipolar format. Specifically, instead of opposing a positive attribute category against a negative attribute category in a single bipolar IAT, separate unipolar IATs can be used in which positive and negative attribute categories are contrasted with neutral attribute categories. In this way, positive and negative implicit alcohol associations can be measured separately in different unipolar task variants. Moreover, in the first study of part two of this thesis, implicit alcohol 
associations with arousal and with sedation were also separately assessed using similar unipolar IAT variants. Findings with unipolar IAT variants indeed demonstrated both negative and positive implicit associations with alcohol. More importantly, positive implicit alcohol associations were found to be related to alcohol use whereas negative implicit alcohol associations were not. Hence, together with findings reported in the first part of this thesis, these results suggest that alcohol use increases as participants hold stronger positive implicit associations with alcohol as indexed by positive unipolar IATs. The size of effects in unipolar negative IATs, on the other hand, appears unrelated to drinking behavior and may reflect recoding processes rather than, or in addition to, implicit alcohol associations to a larger extent than effects in positive unipolar IATs. Further, in line with earlier findings (e.g., Wiers et al., 2002), participants were also found to hold implicit alcohol associations with arousal that were strongly related to drinking behavior. In addition, results also demonstrated implicit alcohol associations with sedation that related to the severity of alcohol-related problems.

Further, in part two of this thesis, it was examined whether results with the IAT would differ depending on the specific target category that the alcohol target category is contrasted with. When implicit associations with alcohol were assessed relative to a different category than soft drinks, which is typically used in alcohol-IAT research, results indicated only a slight impact on IAT effects. However, although testing implicit associations with alcohol relative to different contrast categories can be useful, findings with the IAT still remain relative and, therefore, difficult to interpret. For this reason, implicit evaluative associations with alcohol were also measured using a non-relative variant of the IAT, the Single Target IAT (ST-IAT; Wigboldus et al., 2006), which does not request that the alcohol target category is contrasted with a second target category. Findings demonstrated that the non-relative ST-IAT can yield different results compared to the relative IAT and that both task variants measure different underlying constructs. Further, positive implicit associations with alcohol relative to soft drinks and non-relative positive implicit alcohol associations were found to predict different aspects of drinking behavior.

The research presented in this thesis thus demonstrates that certain features of the IAT procedure may complicate the interpretation of IAT effects and suggests that these limitations of the IAT procedure were to some extent responsible for previous IAT findings in alcohol research. Nevertheless, the present findings also indicate that the IAT, with minor modifications, can yield new insights in the cognitive processes underlying alcohol use. Together, the findings in this thesis indicate that positive and negative implicit associations with alcohol may simultaneously exist. However, whereas positive implicit alcohol associations were found to be related to drinking behavior, negative implicit associations were unrelated to alcohol intake. Thus, these results suggest that positive implicit alcohol associations may play an important role in drinking behavior, in addition to implicit arousal associations with alcohol and explicit cognitive processes. Based on these findings, it is concluded that research should examine ways to change the strength of implicit alcohol associations and their impact on drinking behavior. 


\section{Samenvatting}

Veel mensen kunnen een glaasje alcohol van tijd tot tijd wel appreciëren, net zoals ik. Overmatig alcoholgebruik kan echter leiden tot ernstige gezondheidsproblemen en sociale problemen. Omwille van die reden proberen onderzoekers al geruime tijd te ontdekken welke denkprocessen een rol spelen bij alcoholgebruik en alcoholmisbruik, zodat geschikte behandelingen kunnen ontwikkeld worden om alcoholgebruik te verminderen. Tot enkele jaren geleden werd dergelijk onderzoek grotendeels uitgevoerd met behulp van vragenlijsten die mensen vroegen om zelf redenen aan te geven voor hun alcoholgebruik. Dit soort vragenlijstenonderzoek kent echter een aantal beperkingen en recente ontwikkelingen hebben dan ook geleid tot een nieuwe lijn van onderzoek die zich richt op impliciete of automatische denkprocessen die mogelijk een rol spelen in alcoholgebruik.

Recente ontwikkelingen tonen aan dat verslavingsgevoelig gedrag zoals alcoholgebruik beter begrepen kan worden vanuit het oogpunt van dual-process modellen. Deze modellen stellen dat gedrag doorgaans bepaald wordt door een dynamische wisselwerking tussen twee kwalitatief verschillende denkprocessen. Zo zou gedrag gestuurd worden door zowel snelle, spontane, associatieve, automatische denkprocessen, als door trage, gecontroleerde denkprocessen die gekenmerkt worden door bewuste overwegingen en regels (bv., Deutsch \& Strack, 2006; Evans \& Coventry, 2006; Fazio \& Towles-Schwen, 1999). Bovendien lijkt het zo te zijn dat automatische denkprocessen steeds belangrijker worden naarmate overmatig alcoholgebruik zich ontwikkelt tot een verslaving (bv., Bechara et al., 2006; Deutsch \& Strack, 2006; Evans \& Coventry, 2006). Het is dus belangrijk dat onderzoekers de juiste meetinstrumenten hebben om beide soorten denkprocessen te kunnen meten. Gecontroleerde denkprocessen zijn over het algemeen gemakkelijk vast te stellen met behulp van vragenlijsten. Automatische denkprocessen zijn echter moeilijker te meten omdat men niet altijd inzicht heeft in de automatische processen die gedrag sturen.

De ontwikkeling van zogenaamde indirecte maten was voor vele onderzoekers een doorbraak omdat zij geloofden dat deze maten mogelijk de aangewezen meetinstrumenten zouden kunnen zijn om automatische denkprocessen te meten. Indirecte maten verschillen immers van (directe) vragenlijsten in die zin dat denkprocessen niet gemeten worden aan de hand van zelfrapportage, maar op basis van de prestatie op een computertaak waarin er snel moet gereageerd worden op gepresenteerde stimuli. Om die reden wordt dan ook aangenomen dat indirecte maten mogelijk een goede indicatie kunnen geven van relatief snelle, automatische processen. Bovendien zijn indirecte maten waarschijnlijk ook minder gevoelig voor gecontroleerde reacties omdat er zo snel 
gereageerd moet worden tijdens de taak. Eén van de indirecte maten die de afgelopen jaren het meeste aandacht heeft gekregen is de Impliciete Associatie Taak (IAT; Greenwald, McGhee, \& Schwartz, 1998).

De IAT is een taak waarin stimuli, die één voor één op het computerscherm verschijnen, met twee knoppen op het toetsenbord moeten geclassificeerd worden in twee keer twee categorieën. Hierbij worden er twee targetcategorieën en twee attribuutcategorieën onderscheiden. De targetcategorieën zijn doorgaans concepten waarin de onderzoeker geïnteresseerd is, zoals alcohol tegenover frisdrank. De attribuutcategorieën zijn meestal evaluatieve concepten, zoals positief tegenover negatief. Omdat deelnemers slechts twee knoppen mogen gebruiken om deze stimuli te classificeren in vier categorieën, zitten telkens één targetcategorie en één attribuutcategorie onder dezelfde knop. De twee targetcategorieën en de twee attribuutcategorieën kunnen bovendien op twee verschillende manieren gecombineerd worden. Deelnemers voeren beide combinatietaken vit tijdens verschillende fases van de taak. Tijdens één fase moeten deelnemers dus bijvoorbeeld alcohol en positieve stimuli met de ene knop classificeren en frisdrank en negatieve stimuli met de andere knop. In de tweede fase zit alcohol vervolgens onder dezelfde knop als negatieve stimuli en frisdrank onder de andere knop samen met positieve stimuli. Het achterliggende idee van deze taak is dat deelnemers sneller zullen reageren wanneer de targetcategorieën en de attribuutcategorieën gecombineerd moeten worden in overeenstemming met hun impliciete associaties tussen de targetconcepten en de attribuutconcepten. Als men dus sneller is wanneer alcohol en positieve stimuli met de ene knop geclassificeerd moeten worden en frisdrank en negatieve stimuli met de andere knop, dan wanneer alcohol en negatieve stimuli een knop delen en de andere knop gebruikt moet worden voor frisdrank en positieve stimuli, zou dit betekenen dat deze persoon positieve impliciete associaties heeft met alcohol. Op die manier kan de IAT dus gebruikt worden automatische gevoelens of evaluaties ten opzichte van de targetconcepten te onderzoeken.

In dit proefschrift ligt de nadruk op de IAT als indirecte maat van automatische alcoholgerelateerde denkprocessen. Bevindingen met de IAT van de afgelopen jaren hebben meerdere malen negatieve impliciete associaties met alcohol laten zien, onafhankelijk van hoeveel alcohol men drinkt. Deelnemers waren dus sneller wanneer alcohol en negatieve stimuli onder één knop zaten en frisdrank en positieve stimuli onder de andere knop dan in de omgekeerde combinatie. Dit resultaat was onverwacht aangezien dezelfde deelnemers op vragenlijsten aangaven dat zii positief waren over het drinken van alcohol (bv., Wiers et al., 2002, 2005). Hoewel men zware en lichte drinkers dus weliswaar niet kan onderscheiden op basis van hun impliciete evaluatieve associaties met alcohol, toonde onderzoek aan dat enkel zware drinkers ook impliciete associaties hadden tussen alcohol en gevoelens van opwinding terwijl lichte drinkers dergelijke impliciete alcoholassociaties met opwinding niet lieten zien (bv., Wiers et al., 2002). Samen suggereren deze bevindingen dat alcoholgebruik grotendeels bepaald wordt door 
impliciete alcoholassociaties met opwinding en expliciete positieve cognities met betrekking tot het drinken van alcohol, terwijl impliciete evaluatieve associaties met alcohol geen belangrijke rol lijken te spelen bij het sturen van alcohol gebruik. Het is echter belangrijk om op te merken dat recent onderzoek ook heeft aangetoond dat de IAT een aantal beperkingen heeft die de interpretatie van bevindingen met de IAT kunnen bemoeilijken. Het doel van dit proefschrift was dan ook tweeledig. Ten eerste werd er onderzocht of beperkingen in de procedure van de IAT mogelijk de reden vormden voor eerdere resultaten met de IAT op het gebied van alcohol onderzoek. Ten tweede werd er nagegaan op welke manieren de IAT eventueel verbeterd zou kunnen worden zodat de taak beter geschikt zou zijn om impliciete alcoholgerelateerde denkprocessen te meten.

In het eerste deel van dit proefschrift werd onderzocht in welke mate bevindingen met de alcohol-IAT eerder veroorzaakt zouden kunnen zijn door hercoderingsprocessen dan door impliciete evaluatieve associaties met alcohol. Hercodering verwijst hier naar het fenomeen dat targetstimuli en attribuutstimuli mogelijk geclassificeerd worden op basis van andere eigenschappen dan degene die de onderzoeker in gedachten had. In het geval van de alcohol-IAT zou het bijvoorbeeld kunnen dat deelnemers alle stimuli classificeren op basis van opvallendheid of bekendheid terwij zij eigenlijk targetstimuli zouden moeten classificeren als alcohol of frisdrank en attribuutstimuli als positief of negatief. In hoofdstuk 2 werd aangetoond dat enkel de grootte van IAT effecten, maar niet hun richting, beïnvloed werd wanneer hercoderingsprocessen gemanipuleerd werden door de opvallendheid van de categorieën in de IAT aan te passen. Bovendien lieten de resultaten zien dat de relatie tussen impliciete evaluatieve associaties met alcohol en alcoholgebruik niet werd afgezwakt wanneer er werd gecontroleerd voor hercodering op basis van opvallendheid: sterkere negatieve impliciete associaties met alcohol gingen gepaard met een afname in alcoholgebruik.

Het is echter zo dat hercodering op basis van opvallendheid slechts één van de mogelijke hercoderingsprocessen is die de prestatie op de IAT kunnen beïnvloeden. Daarom werden er in de overige drie hoofdstukken in het eerste deel van dit proefschrift nieuwe varianten van de IAT gebruikt, die hercoderingsprocessen in de taak zouden moeten elimineren of afzwakken, om impliciete evaluatieve associaties met alcohol te meten. In overeenstemming met de hypothese dat eerdere resultaten met de standaard IAT in zekere mate door hercoderingsprocessen werden veroorzaakt, werd er met deze IAT varianten minder ondersteuning gevonden voor negatieve impliciete alcoholassociaties en lieten resultaten zelfs positieve impliciete associaties met alcohol zien. Verder lieten ook alle studies in het eerste deel van dit proefschrift zien dat impliciete evaluatieve associaties met alcohol gerelateerd waren aan alcoholgebruik: sterkere negatieve impliciete alcoholassociaties gingen gepaard met een afname in alcoholgebruik terwiil sterkere positieve impliciete alcoholassociaties gerelateerd waren aan een toename in alcoholgebruik. Deze resultaten suggereren dus ten eerste dat de validiteit van de IAT als maat voor impliciete associaties vergroot kan worden door te controleren voor 
hercoderingsprocessen in de IAT. Ten tweede laten deze bevindingen zien dat impliciete evaluatieve alcoholassociaties wel degelijk een rol spelen in drinkgedrag.

Het tweede deel van dit proefschrift behandelde de dubbele bipolariteit van de categorieën in de IAT. Met dubbele bipolariteit wordt bedoeld dat deelnemers tijdens het vitvoeren van de IAT stimuli moeten indelen in twee keer twee categorieën. Twee van deze categorieën, bijvoorbeeld alcohol en frisdrank, vormen de targetdimensie en de andere twee categorieën, bijvoorbeeld positief en negatief, vormen de polen van de attribuutdimensie. Dit betekent dat zowel de targetdimensie als de attribuutdimensie twee polen hebben en dus bipolair zijn. De bipolariteit van de targetdimensie zorgt ervoor dat de IAT alleen kan aantonen dat alcohol bijvoorbeeld sterker geassocieerd is met negatief dan met positief in vergelijking met frisdrank. De impliciete alcohol associaties gemeten met de IAT zijn dus altiid relatief ten opzichte van een contrastcategorie zoals frisdrank. Hierdoor is het onduidelijk of de IAT impliciete associaties met alcohol reflecteert, of impliciete associaties met frisdrank, of beiden. Het feit dat ook de attribuutcategorieën bipolair zijn maakt het bovendien onmogelijk om te onderzoeken of er tegelijkertijd zowel positieve als negatieve impliciete alcoholassociaties bestaan. Dit vormt een probleem aangezien alcoholgebruik gekenmerkt wordt door een sterke ambivalentie (Conner \& Sparks, 2002).

In de studies in het tweede deel van dit proefschrift werd gebruik gemaakt van varianten van de IAT waarin de attribuutdimensie in een unipolaire vorm werd aangeboden. Dit betekent dat bijvoorbeeld een positieve en een negatieve attribuutcategorie niet langer met elkaar worden gecontrasteerd, zoals in een bipolaire IAT, maar dat er verschillende unipolaire IATs gebruikt worden waarin positieve of negatieve attribuutcategorieën gecontrasteerd worden met neutrale attribuutcategorieën. Op die manier kunnen positieve en negatieve impliciete associaties met alcohol dus afzonderlijk gemeten worden met verschillende unipolaire IAT varianten. Bovendien is het zo ook mogelijk om de relatie van positieve en negatieve impliciete alcoholassociaties met alcoholgebruik afzonderlijk te onderzoeken. Op dezelfde manier werden in het eerste deel van dit proefschrift ook impliciete alcoholassociaties met opwinding en impliciete alcoholassociaties met passiviteit afzonderlijk gemeten met unipolaire IATs.

Zoals verwacht werden er zowel positieve als negatieve impliciete alcoholassociaties gevonden met unipolaire IATs. Bovendien waren positieve impliciete alcoholassociaties gerelateerd aan alcoholgebruik terwijl negatieve impliciete alcoholassociaties geen relatie lieten zien met drinkgedrag. Wanneer deze bevindingen gerelateerd worden aan de bevindingen uit het eerste deel van dit proefschrift, kan gesuggereerd worden dat alcoholgebruik lijkt toe te nemen naarmate men sterkere positieve impliciete associaties heeft met alcohol. Resultaten met negatieve unipolaire IATs blijken daarentegen ongerelateerd aan alcoholgebruik en reflecteren dus mogelijk in sterkere mate hercoderingsprocessen dan impliciete alcoholassociaties in vergelijking met positieve unipolaire IATs. In overeenstemming met eerdere bevindingen (bv., Wiers et al., 2002), 
werd ook aangetoond dat deelnemers impliciete associaties hadden tussen alcohol en opwinding en dat deze impliciete associaties bovendien sterk gerelateerd waren aan drinkgedrag. Verder lieten de resultaten ook impliciete associaties tussen alcohol en passiviteit zien die gerelateerd waren aan de ernst van alcoholgerelateerde problemen.

In het tweede deel van dit proefschrift werd ook onderzocht of resultaten met de IAT verschillen afhankelijk van de targetcategorie die gebruikt wordt als contrast voor de alcohol targetcategorie. Resultaten lieten zien dat het meten van impliciete associaties met alcohol relatief ten opzichte van een andere categorie dan frisdrank, zoals standaard gedaan wordt in alcohol-IAT onderzoek, slechts een kleine impact had op de bevindingen. Het is echter wel belangrijk om op te merken dat het meten van impliciete alcoholassociaties relatief ten opzichte van verschillende contrasterende targetcategorieën wel een bruikbare strategie is, maar dat IAT resultaten nog steeds relatief zijn en bijgevolg moeilijk te interpreteren. Daarom werden impliciete alcoholassociaties ook gemeten met een niet-relatieve IAT variant, namelijk de Single Target IAT (ST-IAT; Wigboldus et al., 2006). In tegenstelling tot de IAT is het in de ST-IAT namelijk niet noodzakelijk om de alcohol targetcategorie te contrasteren met een tweede targetcategorie. De IAT en de STIAT lieten inderdaad verschillende resultaten zien en meten dus mogelijk verschillende constructen. Verder lieten de resultaten ook zien dat positieve impliciete associaties met alcohol relatief ten opzichte van frisdrank en niet-relatieve positieve impliciete alcoholassociaties voorspellend waren voor verschillende aspecten van drinkgedrag.

Het onderzoek in dit proefschrift toont aan dat bepaalde eigenschappen van de IAT procedure de interpretatie van bevindingen met de IAT kunnen bemoeilijken en suggereert dat deze beperkingen van de IAT in elk geval in zekere mate verantwoordelijk waren voor eerdere bevindingen met de IAT in alcoholonderzoek. Echter, de huidige bevindingen laten ook zien dat de IAT, mits voorzien van een aantal kleine aanpassingen, nieuwe inzichten kan verschaffen in de denkprocessen die alcoholgebruik sturen. Zo werd er aangetoond dat positieve en negatieve impliciete alcoholassociaties gelijktijdig kunnen bestaan en dat positieve impliciete alcoholassociaties gerelateerd zijn aan drinkgedrag terwijl negatieve impliciete alcoholassociaties geen relatie vertonen met alcoholgebruik. Deze resultaten suggereren dus dat positieve impliciete alcoholassociaties, net zoals impliciete associaties tussen alcohol en opwinding en expliciete denkprocessen, mogelijk een belangrijke rol spelen in drinkgedrag. Het is dan ook belangrijke dat toekomstig onderzoek zich toelegt op het ontwikkelen van manieren om zowel de sterkte van impliciete alcoholassociaties als hun invloed op gedrag te veranderen. 



\section{References}

Aizen, I. (1991). The theory of planned behavior. Organizational Behavior and Human Decision Processes, 50, 179. 211.

Asendorpf, J. B., Banse, R., \& Mücke, D. (2002). Double dissociation between implicit and explicit personality selfconcept: The case of shy behavior. Journal of Personality and Social Psychology, 83, 380-393.

Baddeley, A. D. (1986). Working Memory. New York: Oxford University Press.

Bandura, A. (1997). Self Efficacy: The Exercise of Control. New York: Freeman.

Banse, R., Seise, J., \& Zerbes, N. (2001). Implicit attitudes towards homosexuality: Reliability, validity, and controllability of the IAT. Zeitschrift für Experimentelle Psychologie, 48, 145-160.

Bargh, J. A., \& Chartrand, T. L. (2000). The mind in the middle: A practical guide to priming and automaticity research. In H. T. Reis (Ed.), (2000). Handbook of Research Methods in Social and Personality Psychology (pp. 253-285). New York: Cambridge University Press.

Baron, R. M., \& Kenny, D. A. (1986). The moderator-mediator variable distinction in social psychological research: Conceptual, strategic and statistical considerations. Journal of Personality and Social Psychology, 51, 1173 1182.

Barrett, L. F., Tugade, M. M., \& Engle, R. W. (2004). Individual differences in working memory capacity and dualprocess theories of the mind. Psychological Bulletin, 130, 553-573.

Bechara, A. (2005). Decision making, impulse control and loss of willpower to resist drugs: A neurocognitive perspective. Nature Neuroscience, 8, 1458-1463.

Bechara, A., Noel, X., \& Crone, E. A. (2006). Loss of willpower: Abnormal neural mechanisms of impulse control and decision making in addiction. In R. W. Wiers \& A. W. Stacy (Eds.), Handbook of Implicit Cognition and Addiction (pp. 215-232). Thousand Oaks, CA: SAGE Publishers.

Beringer, J. (1996). Experimental Run Time System (ERTS), Version 3.18. Frankfurt, Germany: BeriSoft.

Blvemke, M., \& Friese, M. (2006). Do features of stimuli influence IAT effects? Journal of Experimental Social Psychology, 42, 163-176.

Bosson, J. K., Swann, W. B., \& Pennebaker, J. W. (2000). Stalking the perfect measure of implicit self-esteem: The blind men and the elephant revisited? Journal of Personality and Social Psychology, 79, 631-643.

Bradley, M. M. (2000). Emotion and motivation. In J. T. Cacioppo, L. G. Tassinary \& G. G. Berntson (Eds.), Handbook of Psychophysiology (2nd ed., pp. 602-642). New York: Cambridge University Press.

Brown, S. A., Goldman, M. S., Inn, A., \& Anderson, L. R. (1980). Expectations of reinforcement from alcohol: Their domain and relation to drinking patterns. Journal of Consulting and Clinical Psychology, 48, 419-426.

Conner, M., \& Sparks, P. (2002). Ambivalence and attitudes. European Review of Social Psychology, 12, 37-70.

Cooper, M. L. (1994). Motivations for alcohol use among adolescents: Development and validation of a four-factor model. Psychological Assessment, 6, 117-128.

Cooper, M. L., Frone, M. R., Russell, M., \& Mudar, P. (1995). Drinking to regulate positive and negative emotions: A motivational model of alcohol use. Journal of Personality and Social Psychology, 69, 990-1005.

Cunningham, W. A., Preacher, K. J., \& Banaji, M. R. (2001). Implicit attitude measures: Consistency, stability, and convergent validity. Psychological Science, 12, 163-170.

Dasgupta, N., McGhee, D. E., Greenwald, A. G., \& Banaii, M. R. (2000). Automatic preference for white Americans: Eliminating the familiarity explanation. Journal of Experimental Social Psychology, 36, 316-328.

De Houwer, J. (2001). A Structural and process analysis of the Implicit Association Test. Journal of Experimental Social Psychology, 37, 443-451.

De Houwer, J. (2002). The Implicit Association Test as a tool for studying dysfunctional associations in psychopathology: Strengths and limitations. Behavior Therapy and Experimental Psychiatry, 53, 115-133.

De Houwer, J. (2003). The Extrinsic Affective Simon Task. Experimental Psychology, 50, 77-85. 


\section{REFERENCES}

De Houwer, J. (2006). What are implicit measures and why are we using them? In R. W. Wiers \& A. W. Stacy (Eds.), Handbook of Implicit Cognition and Addiction (pp. 11-28). Thousand Oaks, CA: SAGE Publishers.

De Houwer, J. (in press). Comparing measures of attitudes at the procedural and functional level. In R. Petty, R. H. Fazio \& P. Brinol (Eds.), Attitudes: Insights from the New Implicit Measures. Hillsdale, NJ: Erlbaum.

De Houwer, J., Crombez, G., Koster, E. H. W., \& De Beul, N. (2004). Implicit alcohol-related cognitions in a clinical sample of heavy drinkers. Journal of Behavior Therapy and Experimental Psychiatry, 35, 275-286.

De Houwer, J., \& De Bruycker, E. (in press). The identification-EAST as a valid measure of implicit attitudes toward alcohol-related stimuli. Journal of Behavior Therapy and Experimental Psychiatry.

De Houwer, J., Geldof, T., \& De Bruycker, E. (2005). The Implicit Association Test as a general measure of similarity. Canadian Journal of Experimental Psychology, 59, 228-239.

De Houwer, J., \& Moors, A. (in press). How to define and examine the implicitness of implicit measures. In B. Wittenbrink \& N. Schwarz (Eds.), Implicit Measures of Attitudes: Procedures and Controversies. New York: Guilford press.

de Jong, P. J., Wiers, R. W., van de Braak, M., \& Huijding, J. (in press). Using the Extrinsic Affective Simon Test as a measure of implicit attitudes toward alcohol: Relationship with drinking behavior and alcohol problems. Addictive Behaviors.

de Liver, Y., van der Pligt, J., \& Wigboldus, D. (in press). Positive and negative associations underlying ambivalent attitudes. Journal of Experimental Social Psychology.

Deutsch, R., \& Strack, F. (2006). Reflective and impulsive determinants of addictive behavior. In R. W. Wiers \& A. W. Stacy (Eds.), Handbook of Implicit Cognition and Addiction (pp. 45-57). Thousand Oaks, CA: SAGE Publishers.

Egloff, B., \& Schmukle, S. C. (2002). Predictive validity of an Implicit Association Test for assessing anxiety. Journal of Personality and Social Psychology, 83, 1441-1455.

Evans, J. J. (2005). Can executive impairments be effectively treated? In P. W. Halligan \& D. T. Wade (Eds.), Effectiveness of Rehabilitation for Cognitive Deficits (pp. 247-256). New York, NY, US: Oxford University Press.

Evans, J. S. B. T. (2003). In two minds: Dual-process accounts of reasoning. Trends in Cognitive Sciences, 7, $454-$ 459.

Evans, J. S. B. T., \& Coventry, K. (2006). A dual process approach to behavioural addiction: The case of gambling. In R. W. Wiers \& A. W. Stacy (Eds.), Handbook of Implicit Cognition and Addiction (pp. 29-43). Thousand Oaks, CA: SAGE Publishers.

Fadardi, J. S., Cox, W. M., \& Klinger, E. (2006). Individualized versus general measures of implicit cognition. In R. W. Wiers \& A. W. Stacy (Eds.), Handbook of Implicit Cognition and Addiction (pp. 121-133). Thousand Oaks, CA: SAGE Publishers.

Fazio, R. H. (1990). Multiple processes by which attitudes guide behavior: The MODE model as an integrative framework. In M. P. Zanna (Ed.), Advances in Experimental Social Psychology (Vol. 23, pp. 75-109). San Diego, CA: Academic Press.

Fazio, R. H., \& Olson, M. A. (2003). Implicit measures in social cognition: Their meaning and use. Annual Review of Psychology, 54, 297-327.

Fazio, R. H., \& Towles-Schwen, T. (1999). The MODE model of attitude-behavior processes. In S. Chaiken \& Y. Trope (Eds.), Dual-Process Theories in Social Psychology (pp. 97-116). NY / London: Guilford Press.

Fiedler, K., \& Bluemke, M. (2005). Faking the IAT: Aided and unaided response control on the implicit association tests. Basic and Applied Social Psychology, 27, 307-316.

Field, M., Mogg, K., \& Bradley, B. P. (2006). Attention to drug-related cues in drug abuse and addiction: Component processes. In R. W. Wiers \& A. W. Stacy (Eds.), Handbook of Implicit Cognition and Addiction (pp. 151-163). Thousand Oaks, CA: Sage Publishers.

Fleming, M. F., Barry, K. L., \& MacDonald, R. (1991). The alcohol use disorders identification test (AUDIT) in a college sample. The International Journal of the Addictions, 26, $1173-1185$.

Gawronski, B., \& Bodenhausen, G. V. (2006). Associative and propositional processes in evaluation: An integrative review of implicit and explicit attitude change. Psychological Bulletin, 132, 692-731.

Gawronski, B., Hofmann, W., \& Wilbur, C. J. (2006). Are "implicit" attitudes unconscious? Consciousness and Cognition, 15, 485-499. 


\section{REFERENCES}

Goldman, M. S. (1999). Risk for substance abuse: Memory as a common etiological pathway. Psychological Science, 10, 196-198.

Goldman, M. S., Del Boca, F. K., \& Darkes, J. (1999). Alcohol expectancy theory: The application of cognitive neuroscience. In H. T. Blane \& K. E. Leonard (Eds.), Psychological Theories of Drinking and Alcoholism (2nd ed., pp. 203-246). New York: Guilford Press.

Govan, C. L., \& Williams, K. D. (2004). Changing the affective valence of the stimulus items influences the IAT by re-defining the category labels. Journal of Experimental Social Psychology, 40, 357-365.

Greenwald, A. G., McGhee, D. E., \& Schwartz, J. L. K. (1998). Measuring individual differences in implicit cognition: The implicit association test. Journal of Personality and Social Psychology, 74, 1464-1480.

Greenwald, A. G., \& Nosek, B. A. (2001). Health of the Implicit Association Test at age 3. Zeitschrift für Experimentelle Psychologie, 48, 85-93.

Greenwald, A. G., Nosek, B. A., \& Banaii, M. R. (2003). Understanding and using the Implicit Association Test: I. An improved scoring algorithm. Journal of Personality and Social Psychology, 85, 197-216.

Greenwald, A. G., Nosek, B. A., Banaji, M. R., \& Klaver, K. C. (2005). Validity of the salience asymmetry interpretation of the IAT: Comment on Rothermund and Wentura (2004). Journal of Experimental Psychology: General, 134, 420-425.

Grenard, J. L., Ames, S. L., Wiers, R. W., Thush, C., Sussman, S., \& Stacy, A. W. (2006). Working memory moderates the predictive effects of drug-related associations on substance use. Manuscript submitted for publication.

Han, H. A., Olson, M. A., \& Fazio, R. H. (2006). The influence of experimentally-created extrapersonal associations on the Implicit Association Test. Journal of Experimental Social Psychology, 42, 259-272.

Hermans, D., Crombez, G., \& Eelen, P. (2000). Automatic attitude activation and efficiency: The fourth horseman of automaticity. Psychologica Belgica, 40, 3-22.

Hill, A. B., \& Paynter, S. (1992). Alcohol dependence and semantic priming of alcohol related words. Personality and Individual Differences, 13, 745-750.

Hofmann, W., Gawronski, B., Gschwendner, T., Le, H., \& Schmitt, M. (2005). A meta-analysis on the correlation between the Implicit Association Test and explicit self-report measures. Personality and Social Psychology Bulletin, 31, 1369-1385.

Holtgraves, T. (2004). Social desirability and self-reports: Testing models of socially desirable responding. Personality and Social Psychology Bulletin, 30, 161-172.

Houben, K., Rothermund, K. and Wiers, R. W. (2006) Eliminating recoding in the Alcohol-IAT: An application of the IAT-RF. Manuscript submitted for publication.

Houben, K., \& Wiers, R. W. (2006a). A Test of the Salience Asymmetry Interpretation of the Alcohol-IAT. Experimental Psychology, 53, 292-300.

Houben, K., \& Wiers, R. W. (2006b). Are Drinkers Implicitly Positive about Drinking Alcohol? Personalizing the Alcohol-IAT to Reduce Negative Extrapersonal Contamination. Accepted for publication pending revisions.

Houben, K., \& Wiers, R. W. (2006c). Assessing Implicit Alcohol Associations with the Implicit Association Test: Fact or Artifact? Addictive Behaviors, 31, 1346-1362.

Houben, K., \& Wiers, R. W. (2006d). Implicit positive alcohol attitudes as determinants of alcohol consumption: Beyond the soda contrast in the IAT. Manuscript submitted for publication.

Houben, K., \& Wiers, R. W. (2006e). Single-target implicit preferences for beer as determinants of consumption. Manuscript submitted for publication.

Jajodia, A., \& Earleywine, M. (2003). Measuring alcohol expectancies with the Implicit Association Test. Psychology of Addictive Behaviors, 17, 126-133.

Jones, B. T., Corbin, W., \& Fromme, K. (2001). A review of expectancy theory and alcohol consumption. Addiction, 96, 57-72.

Jones, B. T., \& McMahon, J. (1996). A comparison of positive and negative alcohol expectancy and value and their multiplicative composite as predictors of post-treatment abstinence survivorship. Addiction, 91, 89-99.

Karpinski, A., \& Hilton, J. L. (2001). Attitudes and the implicit association test. Journal of Personality and Social Psychology, 81, 774-788.

Karpinski, A., \& Steinman, R. B. (2006). The Single-Category Implicit Association Test as a measure of implicit social cognition. Journal of Personality and Social Psychology, 91, 16-32. 


\section{REFERENCES}

Kim, D.-Y. (2003). Voluntary controllability of the Implicit Association Test (IAT). Social Psychology Quarterly, 66, 83-96.

Lewicki, P., Hill, T., \& Czyzewska, M. (1992). Nonconscious acquisition of information. American Psychologist, 47, 796-801.

McCusker, C. G. (2001). Cognitive biases and addiction: An evolution in theory and method. Addiction, 96, 47-56.

Mierke, J., \& Klaver, K. C. (2001). Implicit association measurement with the IAT: Evidence for effects of executive control processes. Zeitschrift für Experimentelle Psychologie, 48, 107-122.

Mierke, J., \& Klaver, K. C. (2003). Method-specific variance in the Implicit Association Test. Journal of Personality and Social Psychology, 85, $1180-1192$.

Mitchell, J. P., Nosek, B. A., \& Banaji, M. R. (2003). Contextual variations in implicit evaluation. Journal Of Experimental Psychology: General, 132, 455-469.

Miyake, A., Friedman, N. P., Emerson, M. J., Witzki, A. H., \& Howerter, A. (2000). The unity and diversity of executive functions and their contributions to complex "frontal lobe" tasks: A latent variable analysis. Cognitive Psychology, 41, 49-100.

Monteith, M. J., Voils, C. I., \& Ashburn-Nardo, L. (2001). Taking a look underground: Detecting, interpreting, and reacting to implicit racial biases. Social Cognition, 19, 395-417.

Moors, A., \& De Houwer, J. (2006). Automaticity: A conceptual and theoretical analysis. Psychological Bulletin, 132, 297-326.

Nisbett, R. E., \& Wilson, T. D. (1977). Telling more than we can know: Verbal reports on mental processes. Psychological Review, 84, 231-259.

Norman, D. A., \& Shallice, T. (1986). Attention to action: Willed and automatic control of behavior. In R. J. Davidson, G. E. Schwartz \& D. Shapiro (Eds.), Consciousness and Self-Regulation: Advances in Research and Theory (pp. 1-18). New York: Plenum.

Nosek, B. A., \& Banaji, M. R. (2001). The Go/No-Go Association Task. Social Cognition, 19, 625-664.

Nosek, B. A., Greenwald, A. G., \& Banaji, M. R. (2006). The Implicit Association Test at age 7: A methodological and conceptual review. In J. A. Bargh (Ed.), Social Psychology and the Unconscious: The Automaticity of Higher Mental Processes (pp. 265-292). Psychology Press.

Nosek, B. A., \& Hansen, J. J. (2007). The associations in our heads belong to us: Searching for attitudes and knowledge in implicit evaluation. Unpublished manuscript.

Olesen, P. J., Westerberg, H., \& Klingberg, T. (2003). Increased prefrontal and parietal activity after training of working memory. Nature Neuroscience, 7, 75-79.

Olson, M. A., \& Fazio, R. H. (2001). Implicit attitude formation through classical conditioning. Psychological Science, 12, 413-417.

Olson, M. A., \& Fazio, R. H. (2002). Implicit acquisition and manifestation of classically conditioned attitudes. Social Cognition, 20, 89-103.

Olson, M. A., \& Fazio, R. H. (2003). Relations between implicit measures of prejudice: What are we measuring? Psychological Science, 14, 636-639.

Olson, M. A., \& Fazio, R. H. (2004). Reducing the influence of extra-personal associations on the implicit association test: Personalizing the IAT. Journal of Personality and Social Psychology, 86, 653-667.

Olson, M. A., \& Fazio, R. H. (2006). Reducing automatically-activated racial prejudice through implicit evaluative conditioning. Personality and Social Psychology Bulletin, 32, $421-433$.

Orford, J. (2001). Addiction as excessive appetite. Addiction, 96, 15-31.

Ostafin, B. D., Palfai, T. P., \& Wechsler, C. E. (2003). The accessibility of motivational tendencies toward alcohol: Approach, avoidance, and disinhibited drinking. Experimental and Clinical Psychopharmacology, 11, 294301.

Ottaway, S. A., Hayden, D. C., \& Oakes, M. A. (2001). Implicit attitudes and racism: Effects of word familiarity and frequency on the Implicit Association Test. Social Cognition, 19, 97-144.

Palfai, T. P., \& Ostafin, B. D. (2003). Alcohol-related motivational tendencies in hazardous drinkers: Assessing implicit response tendencies using the modified-IAT. Behavior Research and Therapy, 41, $1149-1162$.

Paulhus, D. L. (1984). Two-component models of socially desirable responding. Journal of Personality and Social Psychology, 46, 598-609.

Payne, B. K. (2005). Conceptualizing control in social cognition: How executive functioning modulates the expression of automatic stereotyping. Journal of Personality and Social Psychology, 89, 488-503. 


\section{REFERENCES}

Penke, L., Eichstaedt, J., \& Asendorpf, J. B. (2006). Single attribute Implicit Association Tests (SA-IAT) for the assessment of unipolar constructs: The case of sociosexuality. Experimental Psychology, 53, 283-291.

Poehlman, T. A., Uhlmann, E., Greenwald, A. G., \& Banaji, M. R. (2005). Understanding and using the Implicit Association test: 3 . Meta-analysis of predictive validity. Manuscript submitted for publication.

Rather, B. C., \& Goldman, M. S. (1994). Drinking-related differences in the memory organization of alcohol expectancies. Experimental and Clinical Psychopharmacology, 2, 167-183.

Rather, B. C., Goldman, M. S., Roehrich, L., \& Brannick, M. (1992). Empirical modeling of an alcohol expectancy memory network using multidimensional scaling. Journal of Abnormal Psychology, 101, 174-183.

Robinson, T. E., \& Berridge, K. C. (1993). The neural basis of drug craving: An incentive-sensitization theory of addiction. Brain Research Reviews, 18, 247-291.

Robinson, T. E., \& Berridge, K. C. (2003). Addiction. Annual Review of Psychology, 54, 25-53.

Roßnagel, C. S. (2001). Revealing hidden covariation detection: Evidence for implicit abstraction at study. Journal of Experimental Psychology: Learning, Memory, and Cognition, 27, 1276-1288.

Rothermund, K., Teige-Mocigemba, S., \& Wentura, D. (2006). Minimizing the influence of recoding in the IAT: The IAT-RF. Manuscript submitted for publication.

Rothermund, K., \& Wentura, D. (2001). Figure-ground asymmetries in the Implicit Association Test (IAT). Zeitschrift für Experimentelle Psychologie, 48, 94-106.

Rothermund, K., \& Wentura, D. (2004). Underlying processes in the Implicit Association Test: Dissociating salience from associations. Journal of Experimental Psychology: General, 133, 139-165.

Rothermund, K., Wentura, D., \& De Houwer, J. (2005). Validity of the salience asymmetry account of the IAT: Reply to Greenwald, Nosek, Banaji, and Klaver (2005). Journal of Experimental Psychology: General, 134, 426 430.

Rudman, L. A. (2004). Sources of implicit attitudes. Current Directions in Psychological Science, 13, 79-82.

Rudman, L. A., \& Heppen, J. B. (2003). Sources of implicit attitudes toward smoking. Unpublished Manuscript.

Rudman, L. A., Greenwald, A. G., Melliot, D. S., \& Schwartz, J. L. K. (1999). Measuring the automatic components of prejudice: Flexibility and generality of the Implicit Association test. Social Cognition, 17, 437-465.

Saunders, J. B., Aasland, O. G., Babor, T. F., De la Fuente, J. R., \& Grant, M. (1993). Development of the Alcohol use Disorders Identification Test (AUDIT): WHO collaborative project on early detection of persons with harmful alcohol consumption. Addiction, 88, 791-804.

Shiffrin, R. M., \& Schneider, W. (1977). Controlled and automatic human information processing: II. Perceptual learning, automatic attending and a general theory. Psychological Review, 84, 127-190.

Sobell, L. C., \& Sobell, M. B. (1990). Self-report issues in alcohol abuse: State of the art and future directions. Behavioral Assessment, 12, 77-90.

Spruyt, A., Hermans, D., De Houwer, J., \& Eelen, P. (2002). On the nature of the affective priming effect: Priming of naming responses. Social Cognition, 20, 227-256.

Stacy, A. W. (1995). Memory association and ambiguous cues in models of alcohol and marijuana use. Experimental and Clinical Psychopharmacology, 3, 183-194.

Stacy, A. W. (1997). Memory activation and expectancy as prospective predictors of alcohol and marijuana use. Journal of Abnormal Psychology, 106, 61-73.

Stacy, A. W., Ames, S. L., \& Knowlton, B. J. (2004). Neurologically plausible distinctions in cognition relevant to drug use etiology and prevention. Substance Use \& Misuse, 39, 1571-1623.

Stacy, A. W., Ames, S. L., Sussman, S., \& Dent, C. W. (1996). Implicit cognition in adolescent drug use. Psychology of Addictive Behaviors, 10, 190-203.

Stacy, A. W., Leigh, B. C., \& Weingardt, K. R. (1994). Memory accessibility and association of alcohol use and its positive outcomes. Experimental and Clinical Psychopharmacology, 2, 269-282.

Steffens, M. C. (2004). Is the Implicit Association Test immune to faking? Experimental Psychology, 51, 165-179.

Strack, F., \& Deutsch, R. (2004). Reflective and impulsive determinants of social behavior. Personality and Social Psychology Review, 8, 220-247.

Strayer, D. L., \& Kramer, A. F. (1994). Strategies and automaticity: I. Basic findings and conceptual framework. Journal of Experimental Psychology: Learning, Memory, and Cognition, 20, 318-341.

Swanson, J. E., Rudman, L. A., \& Greenwald, A. G. (2001). Using the implicit association test to investigate attitudebehaviour consistency for stigmatized behaviour. Cognition and Emotion, 15, 207-230. 


\section{REFERENCES}

Teachman, B. A., \& Woody, S. R. (2003). Automatic processing in spider phobia: Implicit fear associations over the course of treatment. Journal of Abnormal Psychology, 112, 100-109.

Teige, S., Schnabel, K., Banse, R., \& Asendorpf, J. (2004). Assessment of multiple implicit self-concept dimensions using the Extrinsic Affective Simon Task (EAST). European Journal of Personality, 18, 495-520.

Thush, C., \& Wiers, R. W. (in press). Explicit and implicit alcohol-related cognitions and the prediction of future drinking in adolescents. Addictive Behaviors.

Thush, C., Wiers, R. W., Ames, S. L., Grenard, J. L., \& Stacy, A. W. (2006). The interaction between working memory and implicit alcohol-related cognition in the prediction of prospective drinking in at-risk youth. Paper presented at the 3rd NWO Cognition Summer School. Doorwerth, The Netherlands. October 13-15, 2006.

Weingardt, K. R., Stacy, A. W., \& Leigh, B. C. (1996). Automatic activation of alcohol concepts in response to positive outcomes of alcohol use. Alcoholism: Clinical and Experimental Research, 20, 25-30.

Wentura, D., \& Rothermund, K. (in press). Paradigms we live by. A plea for more basic research on the IAT. In B. Wittenbrink \& N. Schwarz (Eds.), Implicit Measures of Attitudes: Procedures and Controversies. New York: Guilford Press.

White, H. R., \& Labouvie, E. W. (1989). Towards the assessment of adolescent problem drinking. Journal of Studies on Alcohol, 50, 30-37.

White, H. R., \& Labouvie, E. W. (2000). Longitudinal trends in problem drinking as measured by the Rutgers Alcohol Problem Index. Paper presented at the 23rd Annual Scientific Meeting of the Research Society on Alcoholism. Denver, CO.

Wiers, R. W., Ganushchack, A., Van de Ende, N., Smulders, F. T. Y., \& de Jong, P. J. (2003). Comparing implicit alcohol associations across different RT-measures: The Implicit Association Test (IAT) versus varieties of the Extrinsic Affective Simon Task (EAST). Paper presented at the 15th Annual Convention of the American Psychological Association (APS). Atlanta, GA. May 29-June 1, 2003.

Wiers, R. W., Gunning, W. B., \& Sergeant, J. A. (1998). Do young children of alcoholics hold more positive or negative alcohol-related expectancies than controls? Alcoholism: Clinical and Experimental Research, 22, 1855-1863.

Wiers, R. W., Hoogeveen, K.-J., Sergeant, J. A., \& Gunning, W. B. (1997). High- and low-dose alcohol-related expectancies and the differential associations with drinking in male and female adolescents and young adults. Addiction, 92, 871-888.

Wiers, R. W., Houben, K., Smulders, F. T. Y., Conrod, P. J., \& Jones, B. T. (2006). To drink or not to drink: The role of automatic and controlled processes in the etiology of alcohol-related problems. In R. W. Wiers \& A. W. Stacy (Eds.), Handbook of Implicit Cognition and Addiction (pp. 339-361). Thousand Oaks, CA: SAGE Publishers.

Wiers, R. W., Sergeant, J. A., \& Gunning, W. B. (2000). The assessment of alcohol expectancies in school children: Measurement or modification? Addiction, 95, 737-746.

Wiers, R. W., van de Luitgaarden, J., van den Wildenberg, E., \& Smulders, F. T. Y. (2005). Challenging implicit and explicit alcohol-related cognitions in young heavy drinkers. Addiction, 100, 806-819.

Wiers, R. W., van Woerden, N., Smulders, F. T. Y., \& de Jong, P. J. (2002). Implicit and explicit alcohol-related cognitions in heavy and light drinkers. Journal of Abnormal Psychology, 171, 648-658.

Wigboldus, D. H. J., Holland, R. W., \& van Knippenberg, A. (2006). Single target implicit associations. Unpublished Manuscript.

Wilson, T. D., Lindsey, S., \& Schooler, T. Y. (2000). A model of dual attitudes. Psychological Review, 107, 101-126.

Zack, M., Poulos, C. X., Fragopoulos, F., \& Macleod, C. M. (2003). Effects of negative and positive mood phrases on priming of alcohol words in young drinkers with high and low anxiety sensitivity. Experimental and Clinical Psychopharmacology, 11, 176-185.

Zack, M., Toneatto, T., \& Macleod, C. M. (1999). Implicit activation of alcohol concepts by negative affective cues distinguishes between problem drinkers with high and low psychiatric distress. Journal of Abnormal Psychology, 108, 518-531. 


\section{Dankwoord}

Ik werk erg graag zelfstandig. Sommigen wijten dit aan een extreme vorm van koppigheid, maar dat weiger ik resoluut te geloven (-)). Die zelfstandigheid (of koppigheid) heeft me er echter nooit van weerhouden om hulp te vragen of te aanvaarden wanneer dit nodig was, wat een goede zaak is want ook een proefschrift schrijf je niet alleen. Er zijn dan ook nogal wat mensen die ik hier graag wil bedanken voor hun hulp in de afgelopen jaren.

Allereerst wil ik mijn twee promotoren van harte bedanken. Reinout, bedankt voor je rotsvaste vertrouwen in mij, de goede raad, het snelle naleeswerk, en natuurlijk voor de kansen die je me geboden hebt in de afgelopen jaren. Ik vond het fijn te weten dat ik, ook al liet je me zelfstandig werken aan dit project, altijd bij jou terecht kon wanneer ik hulp nodig had. Anita, bedankt voor je advies en inzicht, de inspirerende gesprekken en steun in de afgelopen jaren. Ik heb van jullie beiden veel bijgeleerd!

Lieve collegaatjes van de drinkgroep, Carolien, Esther, Jade, en Tim, bedankt voor de gezelligheid tijdens ons wekelijks lunchoverleg. Het is tof dat jullie altijd bereid zijn om mee te denken! Tim, ii bent bovendien een keitoffe kamergenoot en ik vind het ontzettend amusant dat je altijd een trucje achter de hand hebt om één of andere computerfrustratie op te lossen. Ik hoop dat we nog vele leuke momenten mogen beleven als kamergenootjes. Lieve Carolien en Esther, jullie wil ik nog extra bedanken voor de leuke babbels en gewoon omdat jullie zulke toffe buren zijn (geweest). Carolien, ontzettend bedankt dat je mijn paranimf wil zijn, want met jou is het altijd gezellig! Remco, bedankt voor de leuke gesprekken, verhelderende discussies, en goede raad. Het is mede dankzii jouw enthousiasme dat ik in het onderzoek verzeild geraakt ben en daarvoor ben ik je zeer dankbaar. Ik ben dan ook heel blij met jou als paranimf! Roy, ex-kamergenootje, bedankt voor de plezante gesprekken en de belevenissen, want met jou in de buurt heb ik me nooit verveeld. Overige EARties en Attimpiërs, bedankt voor de nuttige feedback en de inspirerende discussies. Alle andere collega's van EP, bedankt voor de leuke en gezellige sfeer op de werkvloer.

Linda, ook al ben je in theorie een collega, in de eerste plaats ben je één van mijn allerbeste vriendinnen. Bedankt voor de dagelijkse pauzes met inhoud! Ik vind het fantastisch dat ik met jou kan praten over onderzoek, maar ook over alle andere dingen des levens. We bewandelen al jaren dezelfde weg - eerst psychologie studeren in Maastricht, daarna promoveren aan dezelfde universiteit - en ik hoop dat dit nog vele jaren zo zal zijn (maar dat zit vast wel goed)! Ook mijn andere "Maastrichtse" vriendinnetjes, Eva en Greet, bedankt dat jullie me af en toe wat afleiding kwamen bezorgen! 
Bert D., Bert F., Dirk \& Tamara, Jimmy, Jochen \& Katrien, Kris \& Anke, Peter \& Katrien, lieve vrienden, ook jullie wil ik hier bedanken. Jullie hebben dan wel niet rechtstreeks geholpen bij het tot stand komen van dit proefschrift look al blijven jullie er hardnekkig van overtuigd dat ik jullie als proefpersonen heb gebruikt voor mijn onderzoek), maar jullie hebben mijn leven buiten de universiteit in elk geval een stuk leuker gemaakt. Een dag niet gelachen, is een dag niet geleefd, en mede dankzij jullie heb ik heel wat afgelachen de afgelopen jaren! Bovendien wil ik jullie bedanken voor het inzicht dat alcohol ook gewoon leuk kan zijn :).

Lieve mama en papa, als ik moet beginnen opsommen waarvoor ik jullie dankbaar ben, dan ben ik wel even bezig. In ieder geval wil ik jullie bedanken voor jullie vertrouwen en oneindige steun. Fijn dat ik altijd bij jullie terecht kan voor raad en advies, maar ook gewoon voor de gezelligheid! Tant Jo, ook voor jou een dikke merci voor je steun en omdat je altijd in me hebt geloofd! Overige familieleden, bedankt voor de interesse die jullie de afgelopen jaren hebben getoond voor mijn onderzoeksproject.

Lieve Jochen, natuurlijk wil ik ook jou bedanken voor zoveel dingen: voor je steun, geduld, begrip, en viteraard voor je hulp bij het ontwerpen van de kaft voor dit proefschrift. Bedankt dat je altijd met me hebt meegeleefd en meegedacht, maar bovenal omdat je er altijd voor me bent. Bedankt voor alles! Ik hou van je. 


\section{Curriculum Vitae}

Katrijn Marie Patricia Irma Houben was born on July 5th 1981, in Maaseik, Belgium. In June 1999 she graduated from secondary school (Heilig-Hart College, Lanaken). She started studying Psychology at Maastricht University in September 1999. She graduated in May 2003 and received her master's degree in cognitive psychology. In June 2003 she began her PhD research at the Department of Experimental Psychology, Maastricht University. She currently holds a position as a postdoctoral fellow at the same department.

\section{PUBLICATIONS}

Houben, K., \& Wiers, R. W. (2006). Assessing Implicit Alcohol Associations with the Implicit Association Test: Fact or Artifact? Addictive Behaviors, 31, 1346-1362.

Houben, K., \& Wiers, R. W. (2006). A Test of the Salience Asymmetry Interpretation of the Alcohol-IAT. Experimental Psychology, 53, 292-300.

Houben, K. (2006). Book review of J. Huijding (2006). Automatic affective associations and psychopathology. Maandblad Geestelijke Volksgezondheid, 61, 685-688.

Houben, K., Wiers, R. W., \& Roefs, A. (2006). Reaction Time Measures of SubstanceRelated Associations. In R. W. Wiers \& A. W. Stacy (Eds.), Handbook of Implicit Cognition and Addiction (pp. 91 -104). Thousand Oaks, CA: SAGE Publishers.

Wiers, R. W., Houben, K., Smulders, F. T. Y., Conrod, P. J., \& Jones, B. T. (2006). To drink or not to drink: The role of automatic and controlled processes in the etiology of alcohol-related problems. In R. W. Wiers \& A. W. Stacy (Eds.), Handbook of Implicit Cognition and Addiction (pp. 339-361). Thousand Oaks, CA: SAGE Publishers.

Wiers, R. W., Houben, K., \& de Kraker, J. (in press). Implicit cocaine associations in active cocaine users and controls. Addictive Behaviors.

\section{Submitted manuscripts}

Houben, K., Rothermund, K., \& Wiers, R. W. (2006). Eliminating Recoding in the AlcoholIAT: An Application of the IAT-RF. Manuscript submitted for publication.

Houben, K., \& Wiers, R. W. (2006). Are Drinkers Implicitly Positive about Drinking Alcohol? Personalizing the Alcohol-IAT to Reduce Negative Extrapersonal Contamination. Accepted for publication pending revisions. 
Houben, K., \& Wiers, R. W. (2006). Relative and Single Implicit Attitudes toward Alcohol as Unique Predictors of Drinking Behavior. Manuscript submitted for publication.

Houben, K., \& Wiers, R. W. (2006). Personalizing the Alcohol-IAT with Individualized Stimuli: Relationship with Drinking Behavior and Drinking-Related Problems. Accepted for publication pending revisions.

Houben, K., \& Wiers, R. W. (2006). Single-Target Implicit Preferences for Beer as Determinants of Consumption. Manuscript submitted for publication.

Wiers, R. W., Rinck, M., Kordts, R., Dictus, M., Houben, K., van den Wildenberg, E., Strack, F. (2006). Train Addictive Impulses Away! Assessing and Re-Training Automatic Tendencies to Drink. Manuscript submitted for publication.

\section{Conference Presentations}

Houben, K., \& Wiers, R. W. (2003). Implicit alcohol associations: Investigating the influence of category labels, bipolar targets and attributes and early experiences: setup of a new study. Special interest meeting on the use of indirect measures of attitudes and associations in clinical and health psychology, August 2003, Diksmuide, Belgium (poster).

Houben, K., \& Wiers, R. W. (2004). Implicit alcohol associations: Influence of target category labels and contrast categories in a unipolar IAT. Annual Meeting of the Research Society on Alcoholism, June 2004, Vancouver, Canada (poster). Alcoholism: Clinical and Experimental Research, 28(5-Suppl.), 102A (abstract).

Houben, K., \& Wiers, R. W. (2004). Implicit alcohol associations: influence of target category labels and contrast categories in a unipolar IAT. Special interest meeting on implicit cognition and health behavior, July 2004, Ghent, Belgium (presentation).

Houben, K., \& Wiers, R. W. (2005). Assessing implicit alcohol associations with the single target IAT. Annual Meeting of the Research Society on Alcoholism, June 2005, Santa Barbara, CA, USA (poster presentation). Alcoholism: Clinical and Experimental Research, 29(5-Suppl.), 25A (abstract).

Houben, K., \& Wiers, R. W. (2006). Personalizing the IAT with standard versus individualized stimuli: Extrapersonal contamination reduction or more deliberative processing? Annual Meeting of the Association for Psychological Science, May 2006, New York, NY, USA (poster).

Houben, K., Rothermund, K., \& Wiers, R. W. (2007). Eliminating recoding in the alcoholIAT: An application of the IAT-RF. Annual Meeting of the Society for Personality and Social Psychology, January 2007, Memphis, TN, USA (poster). 